\title{
The period adding and incrementing bifurcations: from rotation theory to applications*
}

\author{
Albert Granados ${ }^{\dagger} \quad$ Lluís Alsedà $\quad$ Maciej Krupa ${ }^{\ddagger}$
}

\begin{abstract}
This survey article is concerned with the study of bifurcations of piecewise-smooth maps. We review the literature in circle maps and quasicontractions and provide paths through this literature to prove sufficient conditions for the occurrence of two types of bifurcation scenarios involving rich dynamics. The first scenario consists of the appearance of periodic orbits whose symbolic sequences and "rotation" numbers follow a Farey tree structure; the periods of the periodic orbits are given by consecutive addition. This is called the period adding bifurcation, and its proof relies on results for maps on the circle. In the second scenario, symbolic sequences are obtained by consecutive attachment of a given symbolic block and the periods of periodic orbits are incremented by a constant term. It is called the period incrementing bifurcation, in its proof relies on results for maps on the interval.

We also discuss the expanding cases, as some of the partial results found in the literature also hold when these maps lose contractiveness. The higher dimensional case is also discussed by means of quasi-contractions. We also provide applied examples in control theory, power electronics and neuroscience where these results can be applied to obtain precise descriptions of their dynamics.
\end{abstract}

Keywords: Piecewise-smooth maps, discontinuous circle maps, period adding, devil's staircase, Farey Tree, period incrementing

\footnotetext{
${ }^{*}$ This work has been partially supported by MINECO-FEDER MTM2012-31714 and MINECO MTM2011-26995-C02-01 Spanish grants.

†algr@dtu,dk, Department of Applied Mathematics and Computer Science, Technical University of Denmark, Building 303B, 2800 Kgns. Lyngby, Denmark.

${ }^{\ddagger}$ Department of Mathematics, Edifici Cc, Universitat Autònoma de Barcelona, 08193 Cerdanyola del Vallès, Spain

$\S$ NeuroMathComp Project-Team, Inria Sophia-Antipolis Research Center, 2004 route des Lucioles, BP93, 06902 Sophia Antipolis cedex, France.
} 


\section{Contents}

1 Introduction 3

2 Basic definitions and overview of results 5

2.1 System definition and properties . . . . . . . . 5

2.2 Overview of the orientation preserving case: the period adding . 10

2.3 Overview of the non-orientable case: the period incrementing . . 12

2.4 Summarizing theorem . . . . . . . . . . . . . . . . 13

3 Orientation preserving case 14

3.1 Detailed description . . . . . . . . . . . . . . . . 14

3.2 Summary of the proof . . . . . . . . . . . . . 17

3.3 Reduction to an orientation preserving circle map and some prop-

erties ....................... 18

3.4 Symbolic dynamics and families of orientation preserving maps . 30

4 Non-orientable case

5 Some remarks on piecewise-smooth expanding maps 42

5.1 Orientable case . . . . . . . . . . . . . . . . 44 43

5.2 Non-orientable case . . . . . . . . . . . . . . . . . . . 44

6 Maximin itineraries and piecewise-smooth maps in $\mathbb{R}^{n}$

6.1 Introduction . . . . . . . . . . . . . . . . . . 45

6.2 Maximin/minimax properties of symbolic sequences . . . . . 45

6.3 Quasi-contractions and piecewise-smooth maps in $\mathbb{R}^{n} \ldots \ldots$. . . 47

6.4 Maximin approach for the one-dimensional case . . . . . . . . . 50

7 Applications 51

7.1 One-dimensional examples . . . . . . . . . . . . . . . . 51

7.1.1 Codimension-two border collision bifurcations . . . . . . . 51

7.1.2 First order sliding-mode controlled system with relays . . 555

7.1.3 Hybrid systems in biology . . . . . . . . . . . 58

7.2 Examples in higher dimensions . . . . . . . . . . . . . 64

7.2.1 Higher order sliding-mode controlled system with relays . 64

7.2.2 ZAD-controlled DC-DC boost converter . . . . . . . . 67

8 Conclusions and future directions

8.1 Period adding for contracting one-dimensional piecewise-smooth

maps . . . . . . . . . . . . . . . 72

8.2 Period incrementing for one-dimensional piecewise-smooth maps 72

8.3 Period adding and incrementing bifurcations in piecewise-smooth expanding maps . . . . . . . . . . . . . . 772

8.4 Maps in higher dimensions . . . . . . . . . . . . . . . . 73 


\section{Introduction}

Piecewise-smooth (piecewise-defined or non-smooth) systems are non-regular or discontinuous systems induced by dynamics associated with sharp changes in position, velocity, or other magnitudes undergoing a jump in their value. This type of systems provide more natural and simpler models in many applications, such as switching systems in power electronics 31, 133, 53, 16, sliding-mode techniques in control theory [130, 43, 52, 47, hybrid systems with resets in neuroscience [35, 83, 103, 74] or impact systems in mechanics [78, 71, 72]. Using non-smooth modeling can reduce the dimension of the system, but may result in more complicated dynamics.

As a consequence of its broad field of application, the interest in such type of systems has considerably grown in the last decade (see [101] for a recent general survey). In particular, piecewise-smooth maps have captured the attention of many researchers, who have studied them from very different perspectives. One of the most reported dynamical aspects are the different bifurcations scenarios that they may exhibit, which turn out to be extraordinarily rich. Seduced by their graphical beauty and mainly supported by computations, many authors have recurrently observed and reported these bifurcation phenomena. However, although they assemble many well known results on circle maps, they have been considered as new and exclusive of piecewise-smooth maps.

In this review article we show that many of the well known results for circle maps developed in the 80's and early 90's can be used to obtain rigorous proofs of general results that can be systematically applied to one-dimensional piecewisesmooth contracting maps. Moreover, we also show how many of these results scattered in the literature are also valid not only for piecewise-smooth expanding maps, but also for higher-dimensional ones.

In the general setting for the one-dimensional case we consider a piecewisesmooth map undergoing a discontinuity at $x=0$, and consider as parameters the two lateral images at this point. To our knowledge, such a map was first studied by Leonov 95, and later on obtained as an approximation of a Poincaré map of smooth flow near a homoclinic bifurcation of the figure eight and butterfly types [120, 80] (see Sections 2.2 and 7.1.1 for more references).

Depending on the signs and magnitude of the slopes of the map at both sides of the discontinuity, the bifurcation scenario in this two-dimensional parameter space may be very different. These signs are determined by the number of twists exhibited by the invariant manifolds (their orientability) involved in the homoclinic bifurcation $(66,80])$. When the map is contracting in both sides of the discontinuity and both slopes have different sign, the so-called period incrementing scenario occurs. This bifurcation was reported in [80, and the details of the proof for this case were given in 18 . However, if both slopes are positive, then the so-called period adding scenario occurs.

Although this latter scenario has been widely reported in the literature (see Section 2.2 its proof is scattered throughout the literature in form of partial results using different approaches. In the late 80's, Gambaudo et al. (61, 60, 63]) provided strong rigorous results in this direction by means of the maximin ap- 
proach (see Section 6). These provide very precise information on the symbolic sequences. Moreover, they were stated for maps in metric spaces of arbitrary dimension, which permits us to adapt them to provide results for piecewisesmooth maps in $\mathbb{R}^{n}$. As a counterpart, they do not allow one to distinguish straightforwardly between the two mentioned bifurcation scenarios.

In order to prove the bifurcation scenario for the increasing-increasing case, we propose to reduce the piecewise-smooth map to a discontinuous circle map. We then assemble and adapt many well known results for rotation theory to the discontinuous case in order to provide the most straight path to prove the period adding bifurcation scenario. The main advantage with respect to the maximin approach is the fact that this method does not require the map to be contracting but it only relies on its invertibility, and hence requires weaker assumptions. However, the maximin approach, although it requires a lot of contractiveness, the obtained results are valid in higher dimensions.

Other approaches suggest to proceed with renormalization arguments [62, 112, 68, 132, 80, 81] to prove the occurrence of the period adding bifurcation scenario.

Beyond its relation with homoclinic bifurcations for flows, such type of bifurcation scenarios have been observed in more applied contexts modeled by both one and $n$-dimensional piecewise-smooth maps. Examples of such applications where these bifurcation scenarios appear are power electronics ([45, 29, 113, 67, 136, 7, 85, 134]), control theory (46, 58, 51, 133, 135, 50, 51]), economics ([126]) or neuroscience $([57,47,48,89,103,122,116,124,125,123)$.

Typically, these bifurcation scenarios are numerically observed in two-dimensional parameter spaces near codimension-two bifurcation points. Such points involve the emergence of an infinite number of bifurcation curves, and were called big bang bifurcations by the non-smooth community [22].

In this survey we also provide illustration on how rotation theory can be applied to provide a rigorous basis to analyze bifurcations of piecewise-smooth maps in four different applied contexts: non-smooth dynamics, control-theory, mathematical neuroscience and power electronics. We first study (Section 7.1.1) bifurcation scenarios around codimension-two bifurcation points given by the collision of two periodic orbits with the boundary (big bang bifucations). In the second example (Section 7.1.2) we consider a nonlinear system subject to sliding-mode control in order to stabilize it around a desired "equilibrium" point. In the third example (Section 7.1.3), we consider a periodically forced integrateand-fire model, a hybrid system widely used in neuroscience. For these two last examples we show how the period adding bifurcation scenario explains the dynamics of the systems and how the symbolic dynamics help to obtain relevant properties from the applied point of view. We finally provide two more examples leading to period-adding like bifurcations for planar piecewise-smooth maps: a higher order system subject to sliding-mode control with relays (Section 7.2.1) and a DC-DC boost converter controlled with so-called ZAD Strategy (Section 7.2.2.

This work is organized as follows. In Section 2 we provide basic definitions 
and a detailed statement of the results for the one-dimensional case. In Section 3 we review and extend results for circle maps to provide a proof of the period adding bifurcation scenario (increasing-increasing or orientation preserving case). A detailed summary of the proof is given in Section 3.2. In Section 5 we emphasize up to which extend the previous results are also valid in the presence of expansiveness.. In Section 4 we revisit the proof provided in [18] for the period incrementing bifurcation scenario (increasing-decreasing or nonorientable case). In Section 6 we review the maximin approach, and show how it can be applied to obtain results for piecewise-smooth maps in $\mathbb{R}^{n}$. Section 7 is dedicated to illustrate how these results can be used in different applied fields by applying them five examples. Finally, we conclude in Section 8 with some discussions and proposals for future directions.

\section{Basic definitions and overview of results}

\subsection{System definition and properties}

Let us consider a piecewise-smooth map

$$
f(x)= \begin{cases}\mu_{\mathcal{L}}+f_{\mathcal{L}}(x) & \text { if } x<0 \\ -\mu_{\mathcal{R}}+f_{\mathcal{R}}(x) & \text { if } x>0\end{cases}
$$

with $x \in \mathbb{R}$ and $f_{\mathcal{L}}, f_{\mathcal{R}}$ smooth functions satisfying

$h .1 f_{\mathcal{L}}(0)=f_{\mathcal{R}}(0)=0$

h. $20<\left(f_{\mathcal{L}}(x)\right)^{\prime}<1, x \in(-\infty, 0)$

h.3 $0<\left|\left(f_{\mathcal{R}}(x)\right)^{\prime}\right|<1, x \in(0, \infty)$.

We wish to describe the possible bifurcation structures obtained when parameters $\mu_{\mathcal{L}}$ and $\mu_{\mathcal{R}}$ are varied.

Remark 2.1. The global contractiveness of the maps $f_{\mathcal{L}}$ and $f_{\mathcal{R}}$ is assumed for simplicity reasons. This allows to state results on bifurcations for arbitrarily large values of $\mu_{\mathcal{L}}$ and $\mu_{\mathcal{R}}$. However, if contractiveness holds only locally at the origin, then all the results presented here are still valid for values of these parameters close enough to the origin. Their validity when contractiveness $i$ lost is discussed in Section 5 .

Remark 2.2. For convenience, we do not define at this point the map $f$ at $x=0$. Roughly speaking, the only difference given by the election of the value of $f$ at $x=0$ will consist of the existence or not of fixed points and periodic orbits at their bifurcation values. We remark that one cannot only consider the value of $f$ at $x=0$ by choosing one lateral image, but also one can consider both images or non. We will focus on this question whenever it becomes relevant. 
Due to condition $h .1$, the map (2.1) is discontinuous at $x=0$ if $\mu_{\mathcal{L}} \neq \mu_{\mathcal{R}}$. As we will show, this discontinuity introduces exclusive dynamical phenomena which are not possible in smooth $\left(C^{1}\right)$ one-dimensional systems. As discussed in the introduction, one observes similar phenomena (the bifurcation scenarios described below) in smooth flows of dimension three near homoclinic bifurcations. They are also observed in smooth maps, when restricted to the circle instead of $\mathbb{R}$.

This discontinuity represents a boundary in the state space abruptly separating two different dynamics: the ones given by the maps $f_{\mathcal{L}}$ and $f_{\mathcal{R}}$. These dynamics will strongly depend on the sign of $f_{\mathcal{R}}^{\prime}(x)$ on $(0, \infty)$ leading to completely different families of periodic orbits. Note that the cases when $f_{\mathcal{L}}(x)$ and $f_{\mathcal{R}}(x)$ have different slopes in their respective domains are conjugate through the symmetry $x \longleftrightarrow-x$. Moreover, as it will be shown below, when both $f_{\mathcal{L}}(x)$ and $f_{\mathcal{R}}(x)$ are decreasing functions in their respective domains, the possible dynamics will be easy. Therefore we can restrict to the case that $f_{\mathcal{L}}(x)$ is an increasing function in $(-\infty, 0)$, as stated in $h .2$.

One of the differences between the families of periodic orbits that one can find depending on the sign of $f_{\mathcal{R}}^{\prime}(x)$ in $(0, \infty)$ will be given by the sequence of steps that periodic orbits perform at each side of the boundary $x=0$. Therefore, we will introduce the symbolic dynamics given by the following symbolic encoding. Given a point $x \in \mathbb{R}$, we associate to its trajectory by $f,\left(x, f(x), f^{2}(x), \ldots\right)$, a symbolic sequence given by

$$
I_{f}(x)=\left(a(x), a(f(x)), a\left(f^{2}(x)\right), \ldots\right),
$$

where

$$
a(x)= \begin{cases}\mathcal{R} & \text { if } x>0 \\ \mathcal{L} & \text { if } x<0\end{cases}
$$

As $a(x)$ provides a symbol of length one $(\mathcal{L}$ or $\mathcal{R})$, one can omit the comas separating the symbols in Equation 2.2 without introducing imprecisions.

We call this the itinerary of $x$ by $f$ or the symbolic sequence associated with the trajectory of $x$ by $f$.

Let us now consider the shift operator acting on symbolic sequences

$$
\sigma\left(\mathbf{x}_{1} \mathbf{x}_{2} \mathbf{x}_{3} \ldots\right)=\left(\mathbf{x}_{2} \mathbf{x}_{3} \ldots\right),
$$

where $\mathbf{x}_{i} \in\{\mathcal{L}, \mathcal{R}\}$.

Clearly, the shift operator satisfies

$$
\sigma\left(I_{f}(x)\right)=I_{f}(f(x)) .
$$

Of special interest for us will be the symbolic sequences associated with periodic orbits. In this case, the symbolic sequences will be also periodic and we will represent them by the repetition of the generating symbolic block. For example, let $\left(x_{1}, x_{2}\right)$ be a periodic orbit,

$$
\begin{aligned}
& f\left(x_{1}\right)=x_{2} \\
& f\left(x_{2}\right)=x_{1},
\end{aligned}
$$


and assume $x_{1}<0$ and $x_{2}>0$. Then, the symbolic sequences associated with $x_{1}$ and $x_{2}$ are

$$
\begin{aligned}
& I_{f}\left(x_{1}\right)=(\mathcal{L} \mathcal{R} \mathcal{L} \mathcal{R} \ldots):=(\mathcal{L} \mathcal{R})^{\infty} \\
& I_{f}\left(x_{2}\right)=(\mathcal{R} \mathcal{L} \mathcal{R} \mathcal{L} \ldots):=(\mathcal{R} \mathcal{L})^{\infty},
\end{aligned}
$$

where $\infty$ indicates infinite repetition.

Due to property (2.5), the shift operator acts on the generating blocks as a cyclic permutation of offset 1 , as it moves the first symbol to the last position. More precisely, if $\left(x_{1}, \ldots, x_{n}\right), x_{i} \in \mathbb{R}$, is a periodic orbit of $f$ and $\left(\mathbf{x}_{1} \ldots \mathbf{x}_{n}\right)^{\infty}$, $\mathbf{x}_{i} \in\{\mathcal{L}, \mathcal{R}\}$, is the symbolic sequence associated with the periodic trajectory of $x_{1}$, then

$$
\sigma\left(I_{f}\left(x_{1}\right)\right)=\left(\mathbf{x}_{2} \mathbf{x}_{3} \ldots \mathbf{x}_{n} \mathbf{x}_{1}\right)^{\infty} .
$$

Hence, a periodic orbit of length $n$ can be represented by $n$ different symbolic sequences obtained by cyclic permutations one from each other.

Definition 2.3. Symbolic sequences can be ordered by lexicographical order induced by $\mathcal{L}<\mathcal{R}$. That is,

$$
\left(\mathbf{x}_{1} \mathbf{x}_{2} \ldots\right)<\left(\mathbf{y}_{1} \mathbf{y}_{2} \ldots\right)
$$

if and only if $\mathbf{x}_{1}=\mathcal{L}$ and $\mathbf{y}_{1}=\mathcal{R}$ or $\mathbf{x}_{1}=\mathbf{y}_{1}$ and there exists some $j \geq 1$ such that

$$
\begin{aligned}
& \mathbf{x}_{i}=\mathbf{y}_{i}, \text { for all } i<j \\
& \mathbf{x}_{j}=\mathcal{L} \\
& \mathbf{y}_{j}=\mathcal{R} .
\end{aligned}
$$

Example 2.4. The sequences $\left(\mathbf{x}_{1}, \mathbf{x}_{2}, \mathbf{x}_{3}, \mathbf{x}_{4}\right)^{\infty}=\left(\mathcal{L}^{2}, \mathcal{R}, \mathcal{L}\right)^{\infty}$ and $\left(\mathbf{y}_{1}, \mathbf{y}_{2}, \mathbf{y}_{3}, \mathbf{y}_{4}\right)^{\infty}=$ $\left(\mathcal{L}^{2}, \mathcal{R}^{2}\right)^{\infty}$ satisfy

$$
\left(\mathcal{L}^{2}, \mathcal{R}, \mathcal{L}\right)^{\infty}<\left(\mathcal{L}^{2}, \mathcal{R}^{2}\right)^{\infty}
$$

as $x_{4}=\mathcal{L}$ and $\mathbf{y}_{4}=\mathcal{R}$ and $\mathbf{x}_{i}=\mathbf{y}_{i}$ for $1 \leq i \leq 3$.

Definition 2.5. Given a periodic symbolic sequence $\mathbf{x}=\left(\mathbf{x}_{1} \ldots \mathbf{x}_{q}\right)^{\infty}$, we will say that it is minimal if

$$
\mathbf{x}=\min _{0 \leq k<q} \sigma^{k}(\mathbf{x}),
$$

and similarly for a maximal symbolic sequence.

Note that the min and max operators act on sequences following the order given in Definition 2.3. and hence its output is also a sequence.

Example 2.6. The sequence $\left(\mathcal{L}^{2}, \mathcal{R}\right)^{\infty}$ is minimal, whereas $\left(\mathcal{R}, \mathcal{L}^{2}\right)^{\infty}$ is maximal, ase we get

$$
\begin{aligned}
\left(\mathcal{L}^{2}, \mathcal{R}\right)^{\infty} & =\min \left\{\left(\mathcal{L}^{2}, \mathcal{R}\right)^{\infty},(\mathcal{L}, \mathcal{R}, \mathcal{L})^{\infty},\left(\mathcal{R}, \mathcal{L}^{2}\right)^{\infty}\right\} \\
\left(\mathcal{R}, \mathcal{L}^{2}\right)^{\infty} & =\max \left\{\left(\mathcal{L}^{2}, \mathcal{R}\right)^{\infty},(\mathcal{L}, \mathcal{R}, \mathcal{L})^{\infty},\left(\mathcal{R}, \mathcal{L}^{2}\right)^{\infty}\right\}
\end{aligned}
$$


Definition 2.7. We will say that a periodic orbit of length $n$ is a $\mathbf{x}_{1} \ldots \mathbf{x}_{n}$ periodic orbit, $\mathbf{x}_{i} \in\{\mathcal{L}, \mathcal{R}\}$, if there exists some point of this periodic orbit, $x_{i} \in \mathbb{R}$ such that

$$
I_{f}\left(x_{i}\right)=\left(\mathbf{x}_{1} \ldots \mathbf{x}_{n}\right)^{\infty} .
$$

Example 2.8. The periodic orbit $(-1,-0.5,1,-2)$, satisfying

$$
f(-1)=-0.5 \quad f(-0.5)=1 \quad f(1)=-2 \quad f(-2)=-1,
$$

is a $\mathcal{L}^{2} \mathcal{R} \mathcal{L}, \mathcal{L} \mathcal{R} \mathcal{L}^{2}, \mathcal{R} \mathcal{L}^{3}$ and $\mathcal{L}^{3} \mathcal{R}$-periodic orbit, as

$$
\begin{aligned}
I_{f}(-1) & =\left(\mathcal{L}^{2} \mathcal{R} \mathcal{L}\right)^{\infty} \\
I_{f}(-0.5) & =\left(\mathcal{L} \mathcal{R} \mathcal{L}^{2}\right)^{\infty} \\
I_{f}(1) & =\left(\mathcal{R} \mathcal{L}^{3}\right)^{\infty} \\
I_{f}(-2) & =\left(\mathcal{L}^{3} \mathcal{R}\right)^{\infty} .
\end{aligned}
$$

Usually, in order to represent the symbolic sequence of a periodic orbit we will choose its minimal representative. For example, assume $\left(x_{1}, \ldots, x_{5}\right)$ is a 5 -periodic orbit such that $I_{f}\left(x_{1}\right)=(\mathcal{L} \mathcal{R} \mathcal{L} \mathcal{L} \mathcal{R})^{\infty}$. Then, we will say that $\left(x_{1}, \ldots, x_{5}\right)$ is a $\mathcal{L}^{2} \mathcal{R} \mathcal{L} \mathcal{R}$-periodic orbit or a periodic orbit of type $\mathcal{L}^{2} \mathcal{R} \mathcal{L} \mathcal{R}$, where the superindex 2 means that there two consecutive symbols $\mathcal{L}$.

Given a periodic orbit, besides its period, one important characteristic associated with its symbolic sequence is the number of symbols $\mathcal{R}$ and $\mathcal{L}$ and how are they distributed along the sequence. The latter will be explained in detail below. For the former we need the following

Definition 2.9. We call $W_{p, q}$ the set of periodic symbolic sequences generated by a symbolic block of length $q$ containing $p$ symbols $\mathcal{R}$ :

$$
W_{p, q}=\left\{\mathbf{y} \in\{\mathcal{L}, \mathcal{R}\}^{\mathbb{N}} \mid \mathbf{y}=\mathbf{x}^{\infty}, \mathbf{x} \in\{\mathcal{L}, \mathcal{R}\}^{q} \text { and } \mathbf{x} \text { contains } p \text { symbols } \mathcal{R}\right\}
$$

Example 2.10. The sets $W_{2,7}$ and $W_{3,7}$ become

$$
W_{2,7}=\left\{\left(\mathcal{L}^{5}, \mathcal{R}^{2}\right)^{\infty},\left(\mathcal{L}^{4}, \mathcal{R}, \mathcal{L}\right)^{\infty}\right\}
$$

and

$$
W_{3,7}=\left\{\left(\mathcal{L}^{4}, \mathcal{R}^{3}\right)^{\infty},\left(\mathcal{L}^{3}, \mathcal{R}^{2}, \mathcal{L}, \mathcal{R}\right)^{\infty},\left(\mathcal{L}^{2}, \mathcal{R}, \mathcal{L}, \mathcal{R}, \mathcal{L}, \mathcal{R}\right)^{\infty}\right\}
$$

Let now $W$ be the set of all periodic symbolic sequences:

$$
W=\bigcup_{(p, q)=1} W_{p, q},
$$

where $(\cdot, \cdot)$ refers to the greatest common divisor.

We next define the $\eta$-number associated with a periodic symbolic sequence: 
Definition 2.11. Let $\mathbf{x}=\left(\mathbf{x}_{1} \ldots \mathbf{x}_{q}\right) \in\{\mathcal{L}, \mathcal{R}\}^{q}$ be a symbolic sequence, and let $p$ be the number of symbols $\mathcal{R}$ contained in $\mathbf{x}$. We then define the $\eta$-number of $\mathbf{x}$ as

$$
\begin{aligned}
\eta: W & \longrightarrow \mathbb{Q} \\
\mathbf{x}^{\infty} & \longmapsto \frac{p}{q}
\end{aligned}
$$

if $\mathbf{x} \in W_{p, q},(p, q)=1$.

Example 2.12. The $\eta$-number of the sequences $\left(\mathcal{R}^{3}, \mathcal{L}\right)^{\infty}$ and $\left(\mathcal{L}^{2}, \mathcal{R}, \mathcal{L}, \mathcal{R}\right)^{\infty}$ become

$$
\eta\left(\left(\mathcal{R}^{3}, \mathcal{L}\right)^{\infty}\right)=\frac{3}{4}
$$

and

$$
\eta\left(\left(\mathcal{L}^{2}, \mathcal{R}, \mathcal{L}, \mathcal{R}\right)^{\infty}\right)=\frac{2}{5} .
$$

As it will detailed below (Section 3.1. see Remark 3.4), under certain conditions, the piecewise-smooth map 2.1 becomes a circle map with rotation number the $\eta$-number. Hence, the $\eta$-number as defined above is frequently referred to as rotation number in the context of piecewise-smooth maps, even when these conditions are not satisfied (see for example [63, 60]).

We now focus on the question of, for a map $f$ of type 2.1 satisfying $h .1-$ $h .3$, what are the possible periodic orbits, their symbolic sequences and their bifurcations in the parameter space given by the offsets, $\mu_{\mathcal{L}} \times \mu_{\mathcal{R}}$.

To this end, we first note that, if $\mu_{\mathcal{L}}, \mu_{\mathcal{R}}<0$, as the maps $f_{\mathcal{L}}$ and $f_{\mathcal{R}}$ are contracting, then $f$ possesses two attracting coexisting $\mathcal{L}$ and $\mathcal{R}$-fixed points $x_{\mathcal{L}}<0$ and $x_{\mathcal{R}}>0$ :

$$
\begin{aligned}
f_{\mathcal{L}}\left(x_{\mathcal{L}}\right) & =x_{\mathcal{L}} \\
f_{\mathcal{R}}\left(x_{\mathcal{R}}\right) & =x_{\mathcal{R}} .
\end{aligned}
$$

The domains of attraction are separated by the boundary $x=0$. Indeed, if both $f_{\mathcal{L}}$ and $f_{\mathcal{R}}$ are increasing maps, then these domains become $(-\infty, 0)$ and $(0, \infty)$, respectively. Note that, although $x=0$ is not an invariant point (an equilibrium), it acts as a separatrix between these domains of attraction.

If one of these two parameters vanishes and becomes positive (for example $\mu_{\mathcal{L}}$ ), the fixed point $x_{\mathcal{L}}$ collides with the boundary $x=0$ and undergoes a border collision bifurcation. Depending on how the map $f$ given in Equation $(2.1)$ is defined at $x=0$, at the moment of the bifurcation this fixed point may still exist or not. Just after this bifurcation, $x_{\mathcal{L}}$ no longer exists, and the fixed point $x_{\mathcal{R}}$ becomes the unique global attractor. As will be discussed below, this fixed point may coexist with a two-periodic orbit. A similar situation occurs when the parameter $\mu_{\mathcal{R}}$ crosses 0 , replacing in the previous argument $x_{\mathcal{L}}$ by $x_{\mathcal{R}}$. Hence, the origin of this parameter space consists of a codimension-two bifurcation point. But then the question arises: what does exist when both parameters are positive and both fixed points disappear in border collision bifurcations? The answer to this question (summarized in Section 2.2 and Section 2.3 depends on the signs of the slopes of the maps $f_{\mathcal{L}}$ and $f_{\mathcal{R}}$ for $x<0$ and $x>0$, respectively. Recalling 


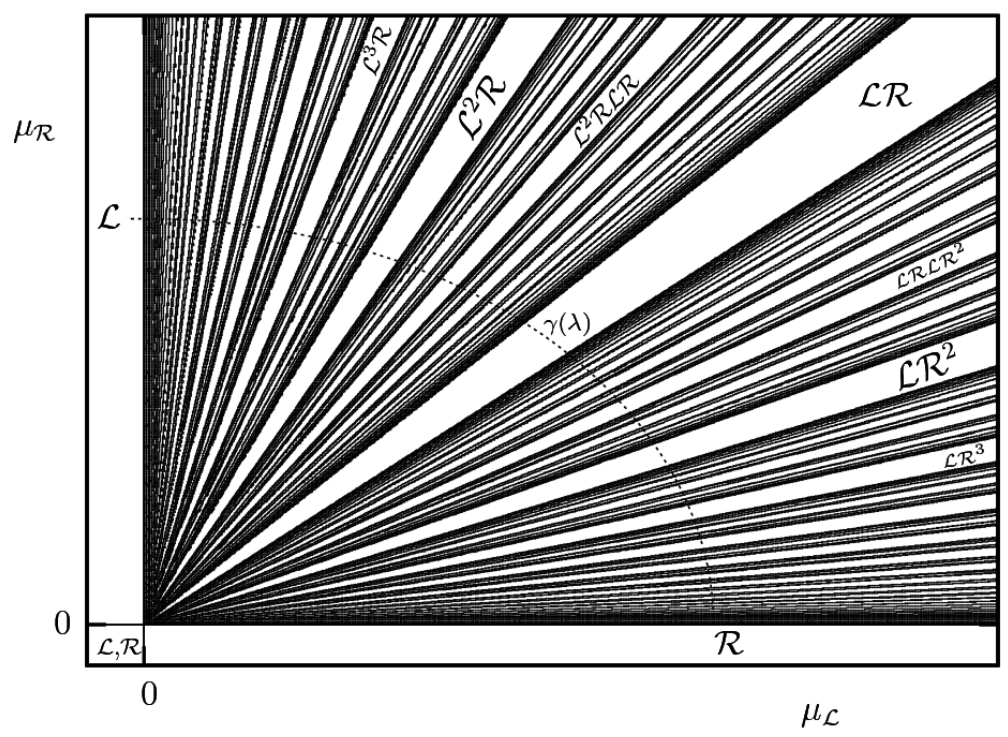

Figure 2.1: Bifurcation curves on the two-dimensional parameter space $\mu_{\mathcal{L}} \times \mu_{\mathcal{R}}$ for a linear increasing-increasing piecewise-smooth map. The periods of the periodic orbits found along the ccurve $\gamma(\lambda)$ are shown in Figure 2.2(a).

that the increasing decreasing and decreasing-cases are conjugate, we will only distinguish between two cases: increasing-increasing and increasing-decreasing. These are also typically referred as orientation preserving and non-orientable cases.

As will be argued in Section 2.4 , the decreasing-decreasing case is straightforward under the assumption of contractiveness.

\subsection{Overview of the orientation preserving case: the pe- riod adding}

The bifurcation scenario when both $f_{\mathcal{L}}$ and $f_{\mathcal{R}}$ are both increasing is shown in Figures 2.1 and 2.2. As shown in Figure 2.1. there exist an infinite number of bifurcation curves emerging from the origin of the parameter space $\mu_{\mathcal{L}} \times \mu_{\mathcal{R}}$. Note that these curves are straight lines due the fact that the chosen map for the simulations was a linear one. Obviously, similar non-linear curves are obtained otherwise. Note also that these curves extend to infinity due to the fact that the chosen maps are globally contracting. If contractiveness was lost, other bifurcations would appear.

The bifurcation curves shown in Figure 2.1 separate regions of existence of periodic orbits. The periods of these periodic orbits are given by "successive 


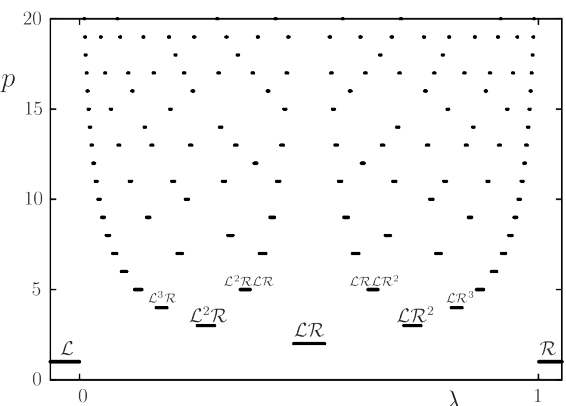

(a)

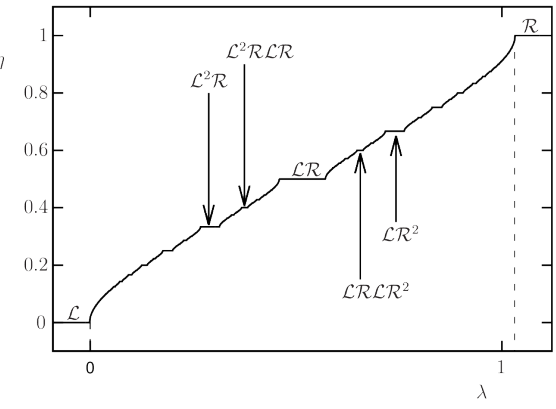

(b)

Figure 2.2: Bifurcation scenario along the curve, $\gamma(\lambda)$, shown in Figure 2.1 and defined in Equation (2.7). (a) periods of the existent periodic orbits. (b) $\eta$-number: ratio between number of $\mathcal{R}$ 's and the period.

addition" of the ones of "neighbouring regions"1 1 , We will make this more precise in Section 3. However, this can be seen in Figure 2.2(a), where we show the periods of the periodic orbits found along the curve shown in Figure 2.1 parametrized counterclockwise by a parameter $\lambda$ which will be clarified in Section 2.4. As one can also see there, the symbolic sequences (some of them are labeled) of obtained periodic orbits are given by successive concatenation of the "neighbouring" ones.

Of relevant interest is the evolution of the $\eta$-number (see Definition 2.11) along the mentioned curve in Figure 2.1. This is shown in Figure 2.2(b), and follows a Devil's staircase (a continuous and monotonically increasing function which is constant locally almost everywhere).

As explained in Section 1, to our knowledge, this bifurcation scenario was first described by Leonov in the late 1950s ( 95, 96, 97, 98]), when studying a piecewise-linear map similar to $(2.1)$ by means of direct computations. Later on, this phenomenon was studied in more detail from different perspectives. It was observed when studying homoclinic bifurcations for flows ([120, 40, 62, 128, 112, 60, 63, 100, 66, 80]) (see Section 7.1.1 for more details), but also in electronic circuits given by the Van der Pol oscillator $([90,99])$ or circle maps [88, 104]. Later on, it was rediscovered by the non-smooth community and called in 22 . period adding. This is precisely defined in Definition 3.5 .

\footnotetext{
${ }^{1}$ The term neighbour refers to the concept of Farey neighbours, see Section 3.1
} 


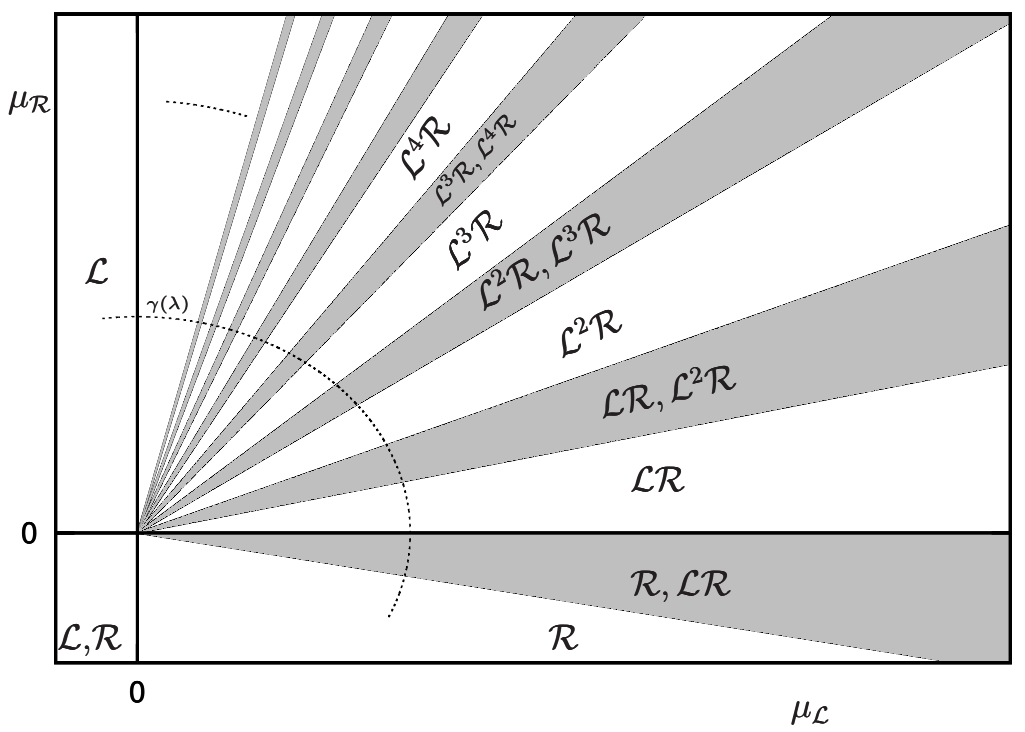

Figure 2.3: Bifurcation curves on the two-dimensional parameter space $\mu_{\mathcal{L}} \times \mu_{\mathcal{R}}$ for a linear increasing-decreasing piecewise-smooth map. Regions of coexistence are filled in gray. The periods of the periodic orbits found along the curve $\gamma(\lambda)$ are shown in Figure 2.4(a).

\subsection{Overview of the non-orientable case: the period in- crementing}

For the increasing-decreasing case, one finds the bifurcation scenario shown in Figures 2.3 and 2.4. As one can see in Figure 2.3. as in the previous case, there exist an infinite number of bifurcation curves emerging from the origin of the parameter space $\mu_{\mathcal{L}} \times \mu_{\mathcal{R}}$. Also as before, the chosen map to perform the simulations was linear and globally contracting. Therefore, the observed bifurcation curves are straight lines extending to infinity. For a non-linear case these lines would becomes non-linear curves and, if contractiveness was lost for larger values of the parameters, new bifurcations would be observed.

Unlike in the orientable case, only families of periodic orbits of the form $\mathcal{L}^{n} \mathcal{R}$ exist for the non-orientable one. Moreover, there exist regions in the parameter space (marked in grey in Figures 2.3 and 2.4 where periodic orbits with symbolic sequences $\mathcal{L}^{n} \mathcal{R}$ and $\mathcal{L}^{n+1} \mathcal{R}$ coexist.

As mentioned in Section 1, this bifurcation scenario was first described by Leonov 95, 96, 97, 98. Later on, it was studied due to its relevance in homoclinic bifurcations involving non-orientable homoclinic manifolds [80, 66]. It was rediscovered in 22] when studying a linear piecewise-smooth map and named period incrementing. Full details proving that the increasing-decreasing case 


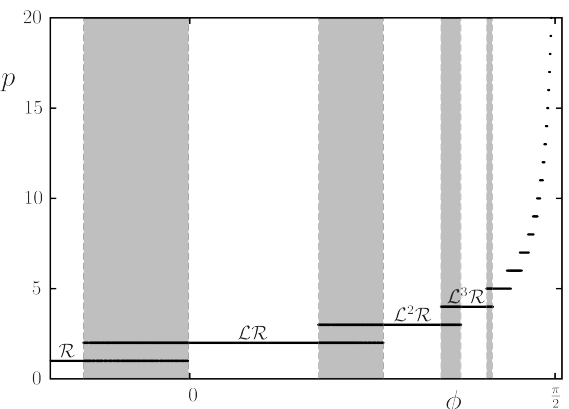

(a)

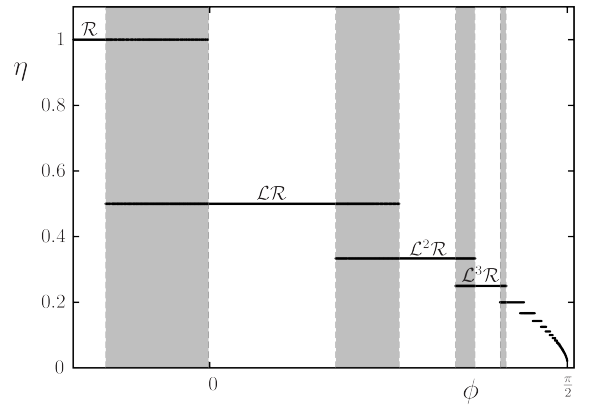

(b)

Figure 2.4: Bifurcation scenario along the curve, $\gamma(\lambda)$, shown in Figure 2.3. (a) periods of the existent periodic orbits. Regions where periodic orbits coexist are filled in gray. (b) $\eta$-number: ratio between number of $\mathcal{R}$ 's and the period.

leads to the occurrence of the period-incrementing bifurcation scenario were given in 18 .

\subsection{Summarizing theorem}

The results presented in the previous sections are summarized in the following

Theorem 2.13. Let $f$ be a map as in Equation (2.1) satisfying conditions h.1h.3. Let $\gamma$ be a $C^{1}$ curve in the parameter space $\mu_{\mathcal{L}} \times \mu_{\mathcal{R}}$ (see Figures 2.1 and 2.3.

$$
\begin{array}{rlc}
\gamma:[0,1] & \longrightarrow & \mathbb{R}^{2} \\
\lambda & \longmapsto & \left(\mu_{\mathcal{L}}(\lambda), \mu_{\mathcal{R}}(\lambda)\right)
\end{array}
$$

satisfying

$H .1 \mu_{\mathcal{L}}(\lambda)>0$ and $\mu_{\mathcal{R}}(\lambda)>0$ for $\lambda \in(0,1)$

H.2 $\left(\mu_{\mathcal{L}}(\lambda)\right)^{\prime}>0$ and $\left(\mu_{\mathcal{R}}(\lambda)\right)^{\prime}<0$ for $\lambda \in[0,1]$

$H .3 \mu_{\mathcal{L}}(0)=0, \mu_{\mathcal{R}}(1)=0$

Then, the bifurcation diagram exhibited by the map $f_{\lambda}$ obtained from Equation (2.1) after performing the reparametrization given by $\gamma$, follows a

i) period adding structure if $0<f_{\mathcal{R}}^{\prime}(x)<1, x \in(0, \infty)$

ii) period incrementing structure if $-1<f_{\mathcal{R}}^{\prime}(x)<0, x \in(0, \infty)$ 
for $\lambda \in[0,1]$.

For a description of the bifurcation scenarios announced in i) and ii), the period adding and period incrementing, see, respectively, Sections 2.2 and 2.3 for an overview, and Sections 3 and 4 for more details and proofs.

We now explain briefly the case when condition $h .2$ is not satisfied $\left(f_{\mathcal{L}}\right.$ is decreasing):

Remark 2.14. If condition $h .2$ is not satisfied and $f_{\mathcal{L}}^{\prime}(x)<0$ in $(-\infty, 0)$, then,

iii) if $f_{\mathcal{R}}^{\prime}(x)<0$ for $(0, \infty)$, only an $\mathcal{L} \mathcal{R}$-periodic orbit can exist for all $\lambda \in$ $(0,1)$,

iv) if $f_{\mathcal{R}}^{\prime}(x)>0$, for $x \in(-\infty, 0)$ the bifurcation scenario is equivalent to ii) but interchanging $\mathcal{L}$ and $\mathcal{R}$ in the symbolic dynamics.

Clearly, iii) comes from the fact that, under these conditions, $f_{\mathcal{L}}((-\infty, 0)) \subset$ $\left(\mu_{\mathcal{L}}, \infty\right)$ and $f_{\mathcal{R}}((0, \infty)) \subset\left(-\infty,-\mu_{\mathcal{R}}\right)$, and hence, due to the contractiveness of these maps, $f$ must possess an $\mathcal{L} \mathcal{R}$-periodic orbit.

The fact that the cases $i v$ ) and ii) are conjugate comes from applying the symmetries given by the change of variables $x \longleftrightarrow-x$.

\section{Orientation preserving case}

\subsection{Detailed description}

We first provide a detailed description of the period adding bifurcation structure by stating some results which will be proved in the rest of this section.

The bifurcation structure given by the so-called period adding is strongly linked with the ordering of the rational numbers given by the Farey tree. In order to explain how this tree is generated, we first define the Farey neighbours. Recall that a rational number $p / q$ is irreducible if $(p, q)=1$.

Definition 3.1. We define the Farey sequence of order $n(n \geq 1), \mathcal{F}_{n}$, as the succession of rational numbers $p / q \in[0,1]$ in ascending order, starting with $0 / 1$ and ending with $1 / 1$, such that $(p, q)=1$ and $q \leq n$.

For example, the Farey sequence of order 6 becomes

$$
\frac{0}{1}, \frac{1}{6}, \frac{1}{5}, \frac{1}{4}, \frac{1}{3}, \frac{2}{5}, \frac{1}{2}, \frac{3}{5}, \frac{2}{3}, \frac{3}{4}, \frac{4}{5}, \frac{5}{6}, \frac{1}{1} .
$$

Note that all rational numbers are contained in $\mathcal{F}_{n}$ for some $n>0$. We then have the following well known result (see [77]).

Theorem 3.2. The following holds:

i) $p / q<r / s$ are Farey neighbours in $\mathcal{F}_{n}$ if, and only if, $r q-p s=1$ 


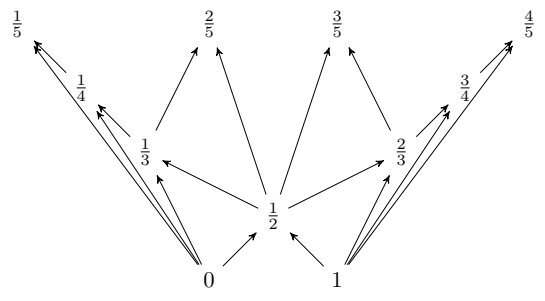

(a)

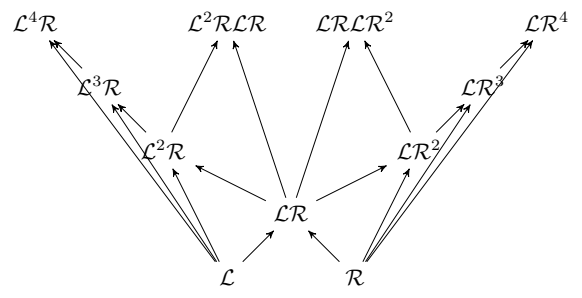

(b)

Figure 3.5: (a) Farey tree of rational numbers. (b) Farey tree of symbolic sequences. Both trees are isomorphic by construction but also through dynamical properties of periodic orbits of orientation preserving circle maps (see Section 3.4.)

ii) if $p / q<r / s$ then

$$
\frac{p}{q}<\frac{p+r}{q+s}<\frac{r}{s} .
$$

Moreover, if $p / q$ and $r / s$ are Farey neighbours at $\mathcal{F}_{n}$ then $p / q<(p+r) /(q+$ $s)$ and $(p+r) /(q+s)<\frac{r}{s}$ are Farey neighbours at $\mathcal{F}_{q+s}$.

Definition 3.3. The fraction $\frac{p+r}{q+s}$ given in Theorem 3.2 is called the Farey sum or mediant of the numbers $\frac{p}{q}$ and $\frac{r}{s}$.

We now construct the Farey tree of rational numbers (see Figure 3.5(a). Starting with the Farey neighbours $0 / 1$ and $1 / 1$, the Farey tree is generated by obtaining rational numbers by adding their numerators and denominators. That is, given two Farey neighbours $p / q$ and $p^{\prime} / q^{\prime}$, they generate the child given by their mediant, $\left(p+p^{\prime}\right) /\left(q+q^{\prime}\right)$, and $p / q$ and $p^{\prime} / q^{\prime}$ become the Farey parents of the rational number $\left(p+p^{\prime}\right) /\left(q^{\prime}+q^{\prime}\right)$. Note that this provides all the Farey sequences $\mathcal{F}_{n}$ and, hence, all rational numbers are found in the Farey tree and are uniquely identified with their Farey parents.

Consder a piecewise-smooth map as defined in Equation (2.1), and recall that we are interested in the simultaneous variation of the parameters $\mu_{\mathcal{L}}$ and $\mu_{\mathcal{R}}$ parametrized by $\lambda \in[0,1]$ through the re-parametrization shown in Equation (2.7). Then we get that, under the conditions i) of Theorem 2.13, when this parameter is varied from 0 to 1 , the periods of these periodic are given by the denominators of the rational numbers numbers given in the Farey tree (see Figure 3.5(a)). 
As noted by some authors [57, other trees, as the Stern-Brocot, can also generate this sequence of periods. However, the most interesting relation between the Farey tree and the sequence of periodic orbits given by the period adding regards their associated symbolic sequences, and not only their periods. To explain this, we recall that the $\eta$-number (Definition 2.11) is given by the ratio between the number of $\mathcal{R}^{\prime} s$ contained in a symbolic sequence and its length.

Remark 3.4. As we will show in Section 3.4 (Corollary 3.32), under conditions i) of Theorem 2.13, the map $f$ given in Equation (2.1) can be reduced to a circle map and the number $\eta$ of a symbolic sequence of a periodic orbit becomes the rotation number of the circle map (see Remark 3.27).

Under conditions $i$ ) of Theorem 2.13, when $\lambda$ in Equation (2.7) is varied from 0 to 1 , one gets periodic orbits with symbolic sequences $\mathbf{x}^{\infty} \in W$, with $\mathbf{x}=$ $\mathbf{x}(\lambda) \in W_{p, q}$. If one then considers the $\eta$-number associated with these symbolic sequences, $\eta(\lambda)$, it turns out that it is a continuous and monotonically increasing function of $\lambda$ whose image are all rational values between 0 and 1 . However, the set of values of $\lambda$ for which $\eta(\lambda)$ is is not defined (it "becomes irrational") forms a Cantor set of zero measure. Hence, $\eta(\lambda)$ is a devil's staircase, as it is continuous, monotonically increasing and locally constant almost everywhere. This function is shown in Figure 2.2(b), and is the well known one formed by the rotation numbers of the periodic orbits of the so-called Arnold circle map

$$
\theta_{n+1}=\theta_{n}+\Omega-\frac{1}{2 \pi} \sin \left(2 \pi \theta_{n}\right)
$$

when $\Omega$ is varied from 0 to 1 .

Note that, for $\eta=0$ and $\eta=1$, the map possesses a fixed points with symbolic sequence $\mathcal{L}$ and $\mathcal{R}$, respectively. These undergo border collision bifurcations for $\lambda=0$ and $\lambda=1$, respectively.

Although each periodic orbit for $\lambda \in(0,1)$ is in one-to-one correspondence with a rational number in the Farey tree through the $\eta$-number, their symbolic sequences are, in principle, not uniquely identified. For example, assume that for a certain value $\lambda=\lambda_{2 / 5}$ there exists a periodic orbit with $\eta=2 / 5$. Its symbolic sequence could be given by any of the generating minimal blocks $\mathcal{L}^{2} \mathcal{R} \mathcal{L} \mathcal{R}$ or $\mathcal{L}^{3} \mathcal{R}^{2}$, as they have length 5 and contain two $\mathcal{R}$ symbols. It turns out that the symbolic sequence of a periodic orbits with $\eta$-number $p / q$ i uniquely determined. To explain this correspondance between periodic orbits and symbolic sequences we construct the Farey tree of symbolic sequences as follows. Starting with the sequences $\mathcal{L}$ and $\mathcal{R}$, one concatenates those sequences whose $\eta$-numbers are Farey numbers. By construction, this provides a unique correspondence between rational numbers and symbolic sequences through their $\eta$-number and the Farey tree. That is, to each rational number $P / Q$ in the Farey tree one associates a symbolic sequence $\Delta$ given by the concatenation

$$
\Delta=\alpha \beta,
$$


where $\alpha$ and $\beta$ are the (minimal) symbolic sequences of the Farey neighbours rational numbers $p / q$ and $p^{\prime} / q^{\prime}$, respectively:

- $p / q<p^{\prime} / q^{\prime}$

- $p^{\prime} q-p q^{\prime}=1$

- $P / Q=\left(p+p^{\prime}\right) /\left(q+q^{\prime}\right)$

As will be discussed in Section 6, this concatenation provides the so-called maximin sequences (see Definition 6.1).

We will prove in Section 3.4 (Propositions 3.31 and 3.33) that the following correspondance holds. The symbolic sequence of the periodic orbit with $\eta$-number $p / q$ is the one which corresponds to rational number $p / q$ in the Farey tree of symblic sequences.

We are now ready to provide a formal description of the period adding bifurcation structure.

Definition 3.5. We say that $f_{\lambda}$, as in Theorem 2.13 i), undergoes a period adding bifurcation structure if

i) for all values of $\lambda$, except for a Cantor set with zero measure, the map $f_{\lambda}$ possesses a unique attracting periodic orbit.

ii) the $\eta$-number, $\eta \in \mathbb{Q}$, as a function of $\lambda$ follows a devil's staircase: it is a monotonically increasing continuous function not defined in a Cantor set of zero measure (it is hence locally constant for almost all values of $\lambda$ )

iii) if $f_{\lambda_{\Delta}}$ possesses a $\Delta$-periodic orbit with $\eta=P / Q,(P, Q)=1$, then there exist values $\lambda_{\alpha}<\lambda_{\Delta}<\lambda_{\beta}$ such that $f_{\lambda_{\alpha}}$ and $f_{\lambda_{\beta}}$ possess $\alpha$ and $\beta$-periodic orbits, respectively, whose $\eta$-numbers are Farey neighbours and their mediant is $P / Q$. Moreover, $\Delta$ is the concatenation of $\alpha$ and $\beta: \Delta=\alpha \beta$

\subsection{Summary of the proof}

In order to facilitate the lecture of the proof of the result described in detail in Section 3.1. we provide a schematic summary of the necessary steps.

1) By performing the change of variables given in Equation (3.1) we first show that a piecewise-defined map $f$ as in (2.1) satisfying $i$ ) in Theorem 2.13 is an orientation preserving circle map (see Definition 3.6). Although this circle map will be discontinuous, in Section 3.3 we will show how classical results for continuous circle maps also hold. In particular, we will show:

(a) Given a map as above, its rotation number exists and is unique (Proposition 3.11.

(b) If a map as above has a rational rotation number, then it possesses a unique and stable periodic orbit (Proposition $3.14+$ contractivness). 
(c) When a map as above has a rational rotation number, the unique periodic orbit must be $p, q$-ordered (it is a twist orbit, see Definition 3.23) (Proposition. 3.24).

2) In Section 3.4 we provide symbolic properties of the itineraries of periodic orbits of orientation preserving circle maps. More precisely:

(a) In Proposition 3.31 we show that the symbolic itinerary of a twist periodic orbit is a $p, q$-ordered symbolic sequence. This identifies each periodic orbit of an orientation preserving map with a unique symbolic itinerary through its rotation number.

(b) In Proposition 3.33 we show that the itineraries of $p$, $q$-ordered periodic orbits are given by concatenation of the itineraries of the periodic orbits with rotation numbers the Farey parents of $p / q$. Hence, they are in the Farey tree of symbolic sequences shown in Figure 3.5(b).

3) In Section 3.4 we also study one-parameter families of orientation preserving (discontinuous) circle maps. This is equivalent to varying the parameter $\lambda$ under the conditions of Theorem $2.13 \mathrm{i}$ ). Using the continuity of the rotation number (Proposition 3.21) and a result of Boyd (Theorem 3.35), we show that the rotation number (and hence the $\eta$-number) follows a devil's staircase leading to the adding scenario when $\lambda$ is varied from 0 to 1 .

We emphasize that the previous steps provide (to our knowledge) the shortest path to prove $i$ ) of Theorem 2.13. However, it is not the only one. In Section 6.4 we provide an alternative to cover some of the steps mentioned above. These involve the concept of maximin sequences and quasi-contractions.

\subsection{Reduction to an orientation preserving circle map and some properties}

In this section we first show that, under condition i) of Theorem 2.13, the piecewise-smooth map (2.1) can be reduced to a class of orientation preserving (increasing) discontinuous circle maps.

Then, we present results on circle maps which are well known in the continuous case. However, by making little modifications of the classical proofs we adapt them to the discontinuous case. We will make this clear in each particular situation.

Let us observe that, under condition $i$ ) of Theorem 2.13 , the piecewisesmooth map (2.1) is increasing on both sides of the discontinuity $x=0$. Hence, all the dynamics are attracted into the interval $\left[f_{\mathcal{L}}(0), f_{\mathcal{R}}(0)\right]=\left[-\mu_{\mathcal{R}}, \mu_{\mathcal{L}}\right]($ see Figure 3.6). By identifying these two values, and considering the circle as $\mathbb{R} /\left[-\mu_{\mathcal{R}}, \mu_{\mathcal{L}}\right]$, the map becomes a circle map which is continuous at $x=0$, but not necessarily at $x=-\mu_{\mathcal{R}} \sim \mu_{\mathcal{L}}$ (see Figure 3.6(a)). As we are interested in varying the parameters $\mu_{\mathcal{R}}$ and $\mu_{\mathcal{L}}$, we perform the change of variables

$$
\begin{aligned}
\phi:\left[-\mu_{R}, \mu_{\mathcal{L}}\right] & \longrightarrow \frac{[0,1]}{x} \\
x & \longmapsto \frac{x+\mu_{\mathcal{R}}}{\mu_{\mathcal{L}}+\mu_{R}},
\end{aligned}
$$




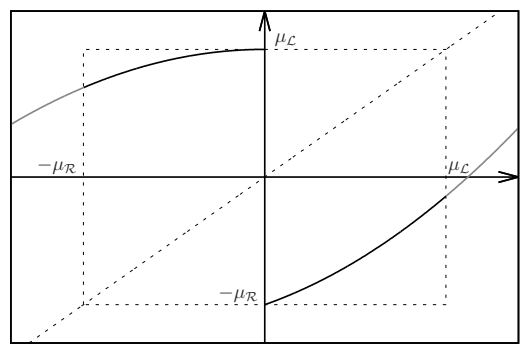

(a)

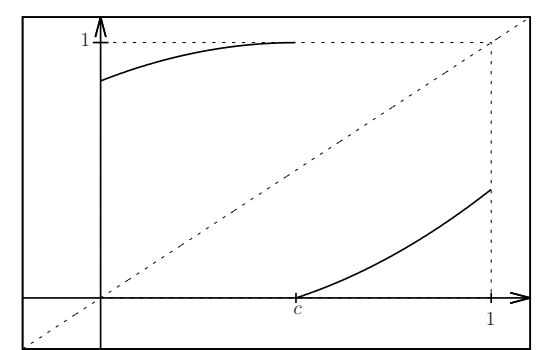

(b)

Figure 3.6: (a) reduction of an increasing-increasing piecewise-smooth map to an orientation preserving circle map. (b) circle map after the change of variables given in Equation (3.1).

which is a strictly increasing homeomorphism mapping $-\mu_{\mathcal{R}}$ to 0 and $\mu_{\mathcal{L}}$ to 1 . Of special interest will be the value

$$
c=\phi(0)=\frac{\mu_{\mathcal{R}}}{\mu_{\mathcal{R}}+\mu_{\mathcal{L}}},
$$

which separates the behaviour given by $\phi \circ f_{\mathcal{L}} \circ \phi^{-1}$ and $\phi \circ f_{\mathcal{R}} \circ \phi^{-1}$.

Hence, by identifying the circle with the interval $[0,1], S^{1}=\mathbb{R} / \mathbb{Z}$, we have reduced the piecewise-smooth map to a class of circle maps, which consists of increasing (orientation preserving) circle maps reaching exactly once the value 1 (degree 1 ) and not necessarily continuous at $x=0 \sim 1$. In the following definition we make precise such class of maps.

Definition 3.6. We say that

$$
f: S^{1} \longrightarrow S^{1}
$$

$S^{1}=\mathbb{R} / \mathbb{Z}$, is an orientation preserving circle map (of degree one) if there exists a unique $c \in[0,1]$ (where $[0,1]$ is identified with the circle $S^{1}$ ) such that

$C .1 f$ is $C^{0}$ in $(0, c)$ and $(c, 1)$

C.2 there exists $c \in[0,1]$ such that $f$ is increasing in $[0, c)$ and $(c, 1]$

C.3 $f\left(c^{-}\right)=1$ and $f\left(c^{+}\right)=0$

C.4 $f\left(0^{+}\right) \geq f\left(1^{-}\right)$, 


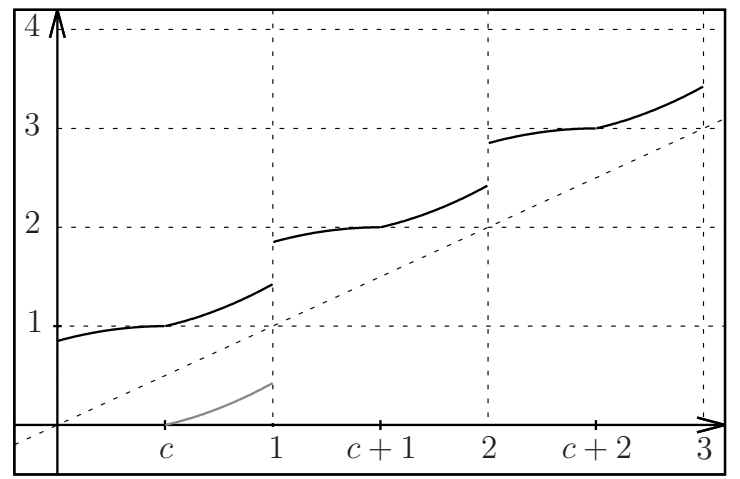

Figure 3.7: Lift of the of the circle map shown in Figure 3.6(b) In gray we show the circle map for $x \in(c, 1)$.

Due to the existence of $c$ fulfilling C.3, such a circle map is of degree one, as the image of $S^{1}$ by $f$ twists at most once around $S^{1}$. When also considering condition $C .4$ we ensure that such an orientation preserving map is invertible. However, condition $C .4$ allows this class of maps to be not necessary continuous at $x=0$. Hence, at $x=0$ and $x=1$ one can choose between the image from the left or right of $x=0$ (or indeed any other value). When convenient, we will choose both values at $x=0$ and $x=1$ and deal with a bi-valued function.

Notice that, at this point, we are not requiring contractiveness, and all results in this section hold also for expansive maps as long as conditions C.1-C.4 are satisfied.

Definition 3.7. Let $f$ be a map satisfying conditions C.1-C.4. We will say that $F$ is a lift of $f$ of degree $N \geq 0$ if

$$
\begin{array}{ll}
F(x+n)=f(x)+n N & \text { if } 0 \leq x<c \\
F(x+n)=f(x)+n N+1 & \text { if } c \leq x<1
\end{array}
$$

If $N=1$, we will refer to $F$ just as the lift of $f$.

Remark 3.8. The previous definition of the degree of a circle map differs from the standard one. When $f$ is a continuous circle map, the degree of its lift $F$ is the integer number $N$ such that

$$
F(x+1)=F(x)+N,
$$

and $F$ is also continuous. In our case, as $f$ is discontinuous at $x=1$, the lift $F$ cannot be continuous and, hence, its degree can be somehow chosen.

Note that if $f$ is a circle map satisfying C.1-C.4 then, if it is continuous at 
$x=0 \sim 1$ and $F$ is its continuous lift, then necessarily $N=1$ and $F$ is of degree one in the classical sense.

From now on, we will restrict to lifts of degree one.

Remark 3.9. Due to condition C.4, the lift of an orientation preserving map is an increasing map, possibly discontinuous at integer numbers, where it undergoes a positive gap.

Remark 3.10. In definition 3.7 the value of the lift $F$ at integer numbers, $n$, is not uniquely defined. When convenient, we will use the one given by $f\left(1^{-}\right)+n$, $f\left(0^{+}\right)+n$ or both. The latter will lead to a bi-valued lift.

The following result is well known and provides the definition of the rotation number of an orientation preserving circle map satisfying C.1-C.4. This was introduced by Poincaré 110] for homeomorphisms of the circle of degree 1 and later studied and extended to rotation intervals by many authors (see [3] and references therein). For discontinuous orientation preserving circle maps, this was proven in [114, 59]. However, if one considers a bi-valued lift at integer numbers (see Remark 3.10), then the standard proof holds.

Proposition 3.11 ([59] III.1.1-1,[114] Theorem 1). Let $f$ be a discontinuous orientation preserving map satisfying C.1-C.4, and let F be its lift. Then, for all $x \in \mathbb{R}$, the limit

$$
\lim _{x \rightarrow \infty} \frac{F^{n}(x)-x}{n}
$$

exists and is independent of $x$.

Proof. We give the standard proof a slight modification to overcome the discontinuities at integer numbers.

As $F$ is increasing, we get that, for $0 \leq x \leq 1$

$$
\frac{F^{n}\left(0^{-}\right)}{n} \leq \frac{F^{n}\left(0^{+}\right)}{n} \leq \frac{F^{n}(x)}{n} \leq \frac{F^{n}\left(1^{-}\right)}{n} \leq \frac{F^{n}\left(1^{+}\right)}{n} .
$$

Noting that we take $F$ bi-valued at integer values, we can write this as

$$
\frac{F^{n}(0)}{n} \leq \frac{F^{n}(x)}{n} \leq \frac{F^{n}(1)}{n},
$$

where $F(0)$ and $F(1)$ can be each of the lateral values.

Using that $F(x+1)=F(x)+1$ and applying it recursively to $F^{n}(1)$ we have that

$$
\frac{F^{n}\left(0^{+}\right)}{n} \leq \frac{F^{n}(x)}{n} \leq \frac{F^{n}\left(1^{+}\right)}{n}=\frac{F^{n}\left(0^{+}\right)+1}{n},
$$

which we can write as

$$
\frac{F^{n}(0)}{n} \leq \frac{F^{n}(x)}{n} \leq \frac{F^{n}(0)+1}{n},
$$


where $F(1)=F(0)+1$ means $F\left(1^{ \pm}\right)=F\left(0^{ \pm}\right)+1$.

Hence, taking limits we get

$$
\lim _{n \rightarrow \infty} \frac{F^{n}(x)-x}{n}=\lim _{n \rightarrow \infty} \frac{F^{n}(x)}{n}=\lim _{n \rightarrow \infty} \frac{F^{n}(0)}{n},
$$

and the limit does not depend on $x$. We next show that, indeed, this limit exists. We apply Proposition 1 of [114, which states that, if a sequence $a_{n}$ satisfies

$$
\left|a_{m+n}-a_{m}-a_{n}\right| \leq A,
$$

for all $n, m \geq 1$ and some constant $A$, then there exists some $\rho$ such that

$$
\left|a_{n}-n \rho\right| \leq A .
$$

The sequence $F^{n}(0)$ satisfies 3.5 with $A=1$. To see this, we use that $F(x+$ $n)=F(x)+n$ to obtain, for any $x \geq 0$,

$$
F^{n}(x)=F^{n}(x \quad(\bmod 1))+[x]<F^{n}(1)+x=F^{n}(0)+x+1,
$$

where $[\cdot]$ denotes the integer part. Then, taking $x=F^{m}(0)$, we get

$$
F^{n+m}(0)<F^{n}(0)+F^{m}(0)+1,
$$

and 3.5 is satisfied with $A=1$. Then, there exists some $\rho$ such that

$$
\lim _{n \rightarrow \infty} \frac{F^{n}(0)}{n} \leq \lim _{n \rightarrow \infty} \frac{1+n \rho}{n}=\rho,
$$

and the limit exists.

Note that this proof differs from the one given in [3]. There, it is first proved that, if $f$ possesses a periodic orbit, then this limit exists and is rational; then it is shown that it does not depend on $x$ as above. Subsequently, the definition is extended to all real numbers using monotonicity and the Dedekind cut construction. In this approach we show the existence of this limit and then we discuss the dynamics of the map depending on its value.

The previous result permits one to define the rotation number of a circle map $f$ fulfilling C.1-C.4. Note that, in general, this depends on the lift of $F$. However, recall that we have restricted to lifts of degree one.

Definition 3.12 (Rotation number). Given a map $f$ satisfying C.1-C.4 and $F$ its lift, we define the rotation number of $f$ as

$$
\rho(f)=\lim _{n \rightarrow \infty} \frac{F^{n}(x)-x}{n},
$$

for any $x \in \mathbb{R}$. 
Remark 3.13. The fact that the limit given in Equation (3.4) exists implies that $F^{n}(x)$ grows linearly with $n$ :

$$
F^{n}(x)-x \sim n \rho(f), n>>1 .
$$

Recalling the properties shown in (3.3), the rotation number $\rho(f)$ can be seen as the average number of times that the lift $F$ crosses an integer number per iteration.

The next result is also standard for continuous circle maps (Proposition 3.7.11 of [3]), it provides the existence of a periodic orbit if the rotation number is rational. Below we prove that it also holds for discontinuous circle maps.

Proposition 3.14. Let $F$ be the lift (of degree one regarding Definition 3.7) of a circle map satisfying C.1-C.4. Then, $\rho(F)=p / q,(p, q)=1$, if and only if there exists $x_{0}$ s.t. $F^{q}\left(x_{0}\right)=x_{0}+p$.

Proposition 3.14, whose proof is given below, is stated assuming that the map $F$ is bi-valued at integer values (see Remark 3.10). This ensures that one always finds a periodic orbit if the rotation number is rational. However, if one considers only one image at integer numbers, given by $f\left(0^{+}\right)$or $f\left(1^{-}\right)$, one can lose the existence of a periodic orbit and get a $\omega$-limit consisting of $q$ points mimicking a periodic orbit. That is, one recovers a result given in [114], which we repeat below for completeness.

Proposition 3.15 ([114] Th. 2). Let $F$ be the lift (of degree one regarding Definition 3.7) of a circle map satisfying C.1-C.4 and $p, q \in \mathbb{Z}$ with $q>0$. If $\rho(F)=p / q$ then exactly one of the following holds.

i) There exists $x_{0} \in \mathbb{R}$ such that $F^{q}\left(x_{0}\right)=x_{0}+p$.

ii) For all $x \in \mathbb{R}, F^{q}(x)>x+p$, and there exists $x_{0} \in \mathbb{R}$ such that

$$
\lim _{x \rightarrow x_{0}^{-}} F^{q}(x)=x_{0}+p .
$$

iii) For all $x \in \mathbb{R}, F^{q}(x)<x+p$, and there exists $x_{0} \in \mathbb{R}$ such that

$$
\lim _{x \rightarrow x_{0}^{+}} F^{q}(x)=x_{0}+p .
$$

Conversely, if either $i)$, ii) or iii) holds then $\rho(F)=p / q$.

Remark 3.16. The situations ii) or iii) occur when $x_{0}=c$. That is, when a periodic orbit bifurcates and the image of $x=0$ by the circle map takes the value on the "wrong" side. More precisely, if $f(0)=f\left(0^{+}\right)$then ii) occurs when a periodic orbits bifurcates when approaching $x=c$ from the left. Similarly, if $f(0)=f\left(1^{-}\right)$, iii) occurs when a periodic orbit bifurcates by colliding with $x=c$ from the right. 
For completeness, we will provide a proof of Proposition 3.14 based on 3 . but adapting it to the discontinuous case, as in the proof of Proposition 3.14. It relies on the following two lemmas.

The first lemma is equivalent to Lemma 2 of [114]. We announce it as in Lemma 3.7.10 of [3] but we adapt its proof to hold also for discontinuous circle maps.

Lemma 3.17. Let $F$ be the lift of a circle map satisfying C.1-C.4, and let $p \in \mathbb{Z}$. Then,

i) If $F^{q}(x)-p>x$ for all $x \in \mathbb{R}$, then there exists $\varepsilon>0$ such that $\rho(F) \geq$ $p / q+\varepsilon$.

ii) If $F^{q}(x)-p<x$ for all $x \in \mathbb{R}$, then there exists $\varepsilon>0$ such that $\rho(F) \leq$ $p / q-\varepsilon$.

Obviously, the proof provided in [114] also holds. However, by considering that $F$ is bi-valued at integer numbers, we can proceed as in the proof for the continuous case [3], which we repeat for completeness.

Proof. We show $i) ;$ ii) can be proven analogously. Note that $G(x)=F^{q}(x)-p-x$ is of degree of zero; i.e. $G(x+1)=G(x)$. Hence, recalling that $F$ is bi-valued at its discontinuities and hence $F^{q}(x)-p-x>0$ holds for each lateral value, it follows that there exists some $\delta>0$ such that, for every $x, F^{q}(x)-p-x \geq \delta$. Then, for all $k$ we get

$$
F^{q k}(x)-x=\sum_{i=0}^{k-1}\left(F^{q}\left(F^{q i}(x)\right)-F^{q i}(x)\right) \geq k(p+\delta) .
$$

Hence, as by Proposition 3.11 the rotation number exists and is unique, we have that

$$
\rho(F)=\lim _{k \rightarrow \infty} \frac{F^{q k}(x)-x}{q k} \geq \frac{k(p+\delta)}{k q}=\frac{p}{q}+\frac{\delta}{q},
$$

which proves $i$ ) with $\varepsilon=\delta / q$.

Note that the proof given in [3] is slightly different, as it does not use the uniqueness of the rotation number.

The following lemma is trivial for the continuous case.

Lemma 3.18 ([114] Lemma 3). Let

$$
F: \mathbb{R} \longrightarrow \mathbb{R}
$$

be a (not necessary continuous) non-decreasing map fulfilling $F(x+1)=x+1$ for all $x \in \mathbb{R}$. Assume that $F\left(x_{1}\right)>x_{1}$ and $F\left(x_{2}\right)<x_{2}$ for some $x_{1}, x_{2} \in \mathbb{R}$. Then there exists some $x_{0}$ such that $F\left(x_{0}\right)=x_{0}$, and $F$ is continuous on the left at $x_{0}$. 
We provide more intuitive proof of this lemma.

Proof. Assume $F(x) \neq x$ for all $x$ and recall that, if $F$ undergoes a discontinuity, the jump must be positive.

Suppose $F(0)>0$. Then the existence of $x_{2}$ implies that either $F$ crosses the diagonal or undergoes a negative jump, either of which gives a contradictions. If $F(0)<0$, then the existence of $x_{1}$ implies that $F$ cannot stay below the diagonal, unless it skips over it by a positive jump. In this case, as $F(1)=$ $F(0)+1<1$, arguing as before we conclude that $F$ has to cross the diagonal.

Remark 3.19. The class of maps considered in Lemma 3.18 is not necessary restricted to lifts of circle maps satisfying C.1-C.4. Note that they may undergo discontinuities with positive jumps between 0 and 1 , whereas the lifts of circle maps satisfying C.1-C.4 are continuous in $(0,1)$.

We now prove Proposition 3.14 by, as before, adapting the proof given in 3 to the discontinuous case.

Proof of Proposition 3.14. Assume that $F^{q}-p$ has a fixed point. Then, we get that $F^{n q}\left(x_{0}\right)-n p=x_{0}$ for all $n>0$, which implies that $\rho(F)=p / q$.

Assume that $\rho(F)=p / q$ and that $F^{q}(x)-p$ does not have a fixed point. Then, by Lemma 3.18, we get that either $F^{q}(x)-p<x$ or $F^{q}(x)-p>x$. But then, by Lemma 3.17, there exists $\varepsilon>0$ such that either $\rho(F)>p / q+\varepsilon$ or $\rho(F)<p / q-\varepsilon$, which is a contradiction.

Note that, besides the fact that we deal with a discontinuous lift, the previous proof differs from the one given in 3 . by the fact that we can use the existence and uniqueness of the rotation number provided by Proposition 3.11 .

Remark 3.20. Proposition 3.14 does not provide the uniqueness nor stability of periodic orbits. However, if, in addition to C.1-C.4, one adds contractiveness, i.e. $f^{\prime}(x)<1$ for $x \in(0, c) \cup(c, 1)$, then one gets that such a periodic orbit is unique and attracting. Note that $f$ might not be differentiable at $x=0 \sim 1$ and $x=c$.

The next result provides the continuity of the rotation number; i.e., if two lifts of orientation preserving maps are "close" (using the uniform norm), so are their rotation numbers. Note that, assuming that these maps are bi-valued at integer values, one can always choose the proper images to properly compare them. Hence, its proof for the discontinuous case becomes the standard one but taking into account this fact and Lemmas 3.17 and 3.18 .

Proposition 3.21 (3 Lemma 3.7.12). The function

$$
F \longmapsto \rho(F)
$$

considered in the space of lifts of circle maps satisfying C.1-C.4 is continuous with the norm of uniform convergence. 
Proof. We proceed as in [3] by adapting the proof to the fact that $F$ is bi-valued in order to to overcome the discontinuities at integer numbers.

Assume $\rho(F) \neq p / q$. Then, the function $G(x)=F^{q}(x)-p-x$ is away from zero. By Lemma 3.18 (applied to $G(x)+x$ ), we get that either $G(x)<0$ or $G(x)>0$ for all $x \in \mathbb{R}$. Then, by Lemma 3.17 , we have that either $\rho(F)<p / q$ or $\rho(F)>p / q$, respectively.

We now note that $G(x)$ has degree 0 ; that is, $G(x+1)=F^{q}(x+1)-p-(x+1)=$ $F^{q}(x)-p-x=G(x)$. Hence, we can ensure that, if $\tilde{G}$ is in small enough neighbourhood of $G$, then either $\tilde{G}<0$ or $\tilde{G}>0$, respectively. This implies that, if $\tilde{F}$ is in a small enough neighbourhood of $F$, then either $\rho(\tilde{F})<p / q$ or $\rho(\tilde{F})>p / q$, respectively.

Hence, we have shown that, if there exist $p_{1} / q_{1}$ and $p_{2} / q_{2}$ such that

$$
\frac{p_{1}}{q_{1}}<\rho(F)<\frac{p_{1}}{q_{1}},
$$

and $\tilde{F}$ is sufficiently close to $F$, then

$$
\frac{p_{1}}{q_{1}}<\rho(\tilde{F})<\frac{p_{2}}{q_{2}},
$$

and hence $F \longmapsto \rho(F)$ is continuous.

The next Lemma shows that the rotation number is increasing as a function of $F$.

Lemma 3.22. Let $f$ and $g$ be two circle maps satisfying C.1-C.4, and let $F$ and $G$ be their lifts, respectively. If $F \geq G$ then $\rho(f) \geq \rho(g)$.

Proof. Let $x<c$ and $F$ and $G$ be two lifts of $f$ and $g$ such that $F(x+1)=$ $F(x)+1$ and $G(x+1)=G(x)+1$. As $F$ and $G$ are increasing functions undergoing a positive gap at $x=k, F^{n}(x)>G^{n}(x)$, even if, for some $n, F^{n}(x)$ or $G^{n}(x)$ reach any of the discontinuities at $x=k$. Hence,

$$
\frac{F^{n}(x)-x}{n} \geq \frac{G^{n}(x)-x}{n} .
$$

As the rotation number does not depend on $x$, we get $\rho(f) \geq \rho(g)$.

We next study properties of the periodic orbits of orientation preserving circle maps satisfying C.1-C.4. These will be crucial to show symbolic properties of periodic orbits in Section 3.4. To this end, we provide the following definitions.

Definition 3.23 (Twist and $p, q$-ordered lifted cycle). Let $f$ be a circle map and $x_{i} \in S^{1}=\mathbb{R} / \mathbb{Z}$ such that

$$
0<x_{0}<x_{1}<\cdots<x_{q-1}<1
$$

and $f^{q}\left(x_{i}\right)=x_{i}, 0 \leq i \leq q-1$. Consider the projection

$$
\begin{aligned}
\Pi: \mathbb{R} & \longrightarrow S^{1}=\mathbb{R} / \mathbb{Z} \\
x & \longmapsto x \quad(\bmod 1)
\end{aligned}
$$


and the lifted orbit or lifted cycle

$$
S=\left\{\Pi^{-1}\left(x_{i}\right)\right\},
$$

which consists of adding $\mathbb{Z}$ to the orbit (3.6). Let $F$ be the lift of $f$. We say that $S$ is a twist lifted cycle or orbit of $F$ if $F$ restricted to $S$ is increasing. If $S$ is a twist lifted cycle of $f$ and

$$
\lim _{n \rightarrow \infty} \frac{F^{n}(x)-x}{n}=\frac{p}{q}
$$

for all $x \in S$, we will say that $S$ is a p,q-ordered lifted cycle of $F$ and $x_{0}<$ $\cdots<x_{q-1}$ is a p,q-ordered cycle or periodic orbit of $f$.

The following result gives us a relation between the spatial and dynamical ordering of a $p, q$-ordered lifted cycle.

Proposition 3.24. Let $0<x_{0}<\cdots<x_{q-1}$ be a p,q-ordered cycle of the circle map $f$, let $F$ be its lift and $S$ the corresponding lifted cycle. Assume that $S$ is given by

$$
S=\left\{x_{0}<\cdots<x_{q-1}<x_{q}<\cdots<x_{2 q-1}<\cdots\right\}
$$

with $x_{j}=x_{i}+n$ if $j=i+n q, 0 \leq i \leq q-1$. Then,

$$
\begin{aligned}
F\left(x_{i}\right) & =x_{i+p} \\
F^{q}\left(x_{i}\right) & =x_{i}+p .
\end{aligned}
$$

The proof of this result is as in the continuous case (3] Lemma 3.7.4) but taking into account that the lift $F$ is bi-valued at integer numbers. We include it here for completeness.

Proof. By properly choosing the image of $F$ at integer values, the lift $F$ restricted to the lifted cycle of the periodic orbit is an order preserving bijection. That is, by iterating the points $x_{0}<\cdots<x_{q-1}$ one visits all the points of the lifted cycle exactly once, and the order is preserved $\left(F\left(x_{j}\right)<F\left(x_{i}\right)\right.$ iff $\left.x_{j}<x_{i}\right)$. Hence, $F\left(x_{i}\right)=x_{i+r}$ for all $i$ and some integer $r$. If not, then one gets that $F\left(x_{j}\right)=x_{j+r_{1}}$ and $F\left(x_{i}\right)=x_{i+r_{2}}$. If $r_{1} \neq r_{2}$, then the number of points of the lifted orbit in $\left[x_{j+r_{1}}, x_{i+r_{2}}\right]$ and in $\left[x_{j}, x_{i}\right]$ does not coincide, and hence the order cannot be preserved.

As the rotation number is $\rho(F)=p / q$, we have that necessarily $x_{i}+p=$ $F^{q}\left(x_{i}\right)=x_{i+q r}$. As $x_{i}$ belongs to a $q$-periodic cycle, we get $x_{i+q r}=x_{i}+r$, which is what we wanted to show.

The next result is also very well known for continuous circle maps. As in previous results, we provide a standard proof adapted for the discontinuous case.

Proposition 3.25. Let $f$ be a circle map satisfying C.1-C.4 and assume that $\rho(f) \in \mathbb{R} \backslash \mathbb{Q}$. Then, if $f$ is contracting $\left(f^{\prime}(x)<1, x \in(0, c) \cup(c, 1)\right)$ and condition $C .4$ is a strict inequality, the $\omega$-limit set of the circle is a Cantor set. 


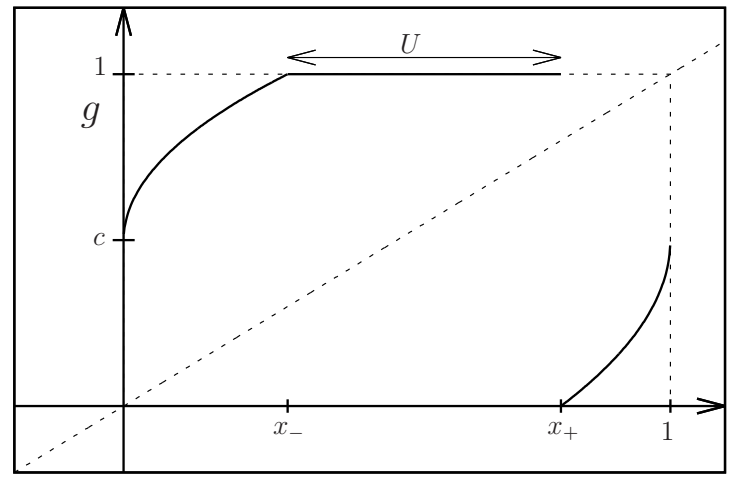

Figure 3.8: Inverse of an orientation preserving circle map satisfying C.1-C.4 with a flat part. See Equation 3.10

Proof. Recall that, if $f\left(0^{+}\right)>f\left(1^{-}\right)$, then $f$ is invertible but not injective. Let $U=\left(x_{-}, x_{+}\right)$with $x_{-}=f\left(1^{-}\right)$and $x_{+}=f\left(0^{+}\right)$. We first show that, if $\rho(f)$ is irrational, then $c \notin f^{n}(U)$ for all $n \geq 1$. As $f$ is invertible in $S^{1} \backslash U$, we consider $f^{-1}$, which is a function with a "hole", as it is not defined in $U$. After filling this hole with the value 1 , we obtain the continuous function

$$
g(y)= \begin{cases}f^{-1}(y) & \text { if } y \in\left[0, x_{-}\right] \\ 1 & \text { if } y \in\left[x_{-}, x_{+}\right] \\ f^{-1}(y) & \text { if } y \in\left[x_{+}, 1\right]\end{cases}
$$

(see Figure 3.8) which has the same rotation number as $f$ with different sign. Note that

$$
\begin{aligned}
g(U) & =1 \\
g(1) & =c .
\end{aligned}
$$

Hence, if for some $n, c \in f^{n}(U)$, then $g^{n}(c) \in U, g^{n+1}(c)=1, g^{n+2}(c)=c$, and hence $g$ has a periodic orbit of period $n+2$, which is not compatible with having irrational rotation number (see Proposition 3.14).

Next we show that

$$
\bigcap_{n \geq 0} f^{n}\left(S^{1}\right)=S^{1} \backslash \bigcup_{i \geq 0} f^{i}(U)
$$

is a Cantor set. As long as $c \notin f^{n}(U)$, at each iteration, $f^{n}\left(S^{1}\right)=S^{1} \backslash f^{n-1}(U)$ consists of subtracting a nonempty interval to the interior of $S^{1} \backslash f^{n-1}(U)$ (see Figure 3.9). Due to the contraction of $f$, the length of the subtracted interval tends to 0 . Moreover, by Corollary 3.3 of [131, the total removed amount, 


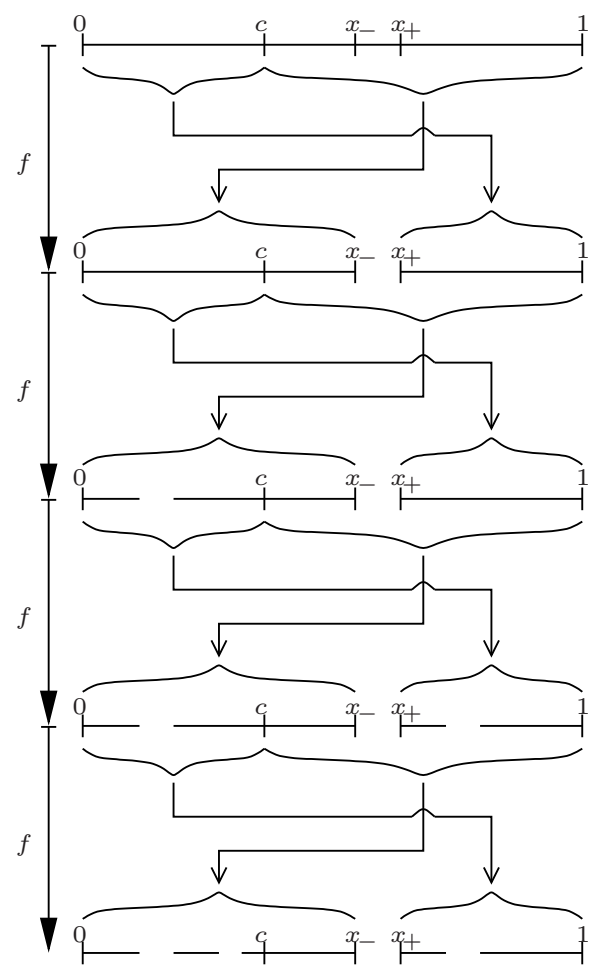

Figure 3.9: Cantor set generated by iterating $U$ : new "holes" are created as long as $c \notin f^{n}(U)$.

$\cup_{i>0} f^{i}(U)$, is dense. This comes from the fact that, although the map $g$ is not differentiable, Denjoy theorem holds and, if the rotation number is irrational, $g$ has no "homtervals"; in particular, $U$ is not a homterval and the sequence $g^{n}(U)$ is pairwise disjoint. Therefore, by construction, $S^{1} \backslash \cup_{i \geq 0} f^{i}(U)$ is a Cantor set. Moreover, also by construction, the images of $x_{-}$and $x_{+}$are dense in this set. Thus, every point in $S^{1} \backslash \cup_{i \geq 0} f^{i}(U)$ has a dense orbit and hence this set becomes the $\omega$-limit of $f$.

Remark 3.26. If condition C.4 is satisfied by an equality (the map becomes continuous) and the rotation number is irrational, then the $\omega$-limit of $f$ may also be a Cantor set or the whole circle. If $f$ is $C^{2}$, then Denjoy theorem holds and the latter occurs. However, if it is $C^{1}$ or $C^{0}, f$ may become a Denjoy counterexample (see [107]), and it's $\omega$-limit may be a Cantor set.

To conclude this section, we recover a piecewise-smooth map as defined in Equation 2.1. As mentioned above, after applying the change of variables 
given in Equation (3.1), the map $\bar{f}(x)=\phi \circ f \circ \phi^{-1}(x)$ becomes an orientationpreserving map satisfying C.1-C.4 with

$$
c=\phi(0)=\frac{\mu_{\mathcal{R}}}{\mu_{\mathcal{R}}+\mu_{\mathcal{L}}} .
$$

After applying the reparameterization $\gamma$ given in Equation (2.7), the value

$$
c_{\lambda}=\frac{\mu_{\mathcal{R}}(\lambda)}{\mu_{\mathcal{R}}(\lambda)+\mu_{\mathcal{L}}(\lambda)}
$$

becomes an strictly decreasing function of $\lambda$ such that

$$
\begin{aligned}
\lim _{\lambda \rightarrow 0^{+}} c_{\lambda} & =1 \\
\lim _{\lambda \rightarrow 1^{-}} c_{\lambda} & =0 .
\end{aligned}
$$

Moreover, for $\lambda=0$ and $\lambda=1$, the map $\bar{f}$ possesses fixed points at $x=1$ and $x=0$, respectively.

Given a piecewise-smooth map $f$ satisfying $h .1-h .3$, we will define its rotation number as the rotation number of the map $\bar{f}=\phi \circ f \circ \phi^{-1}$ obtained after a reduction to a circle map:

$$
\rho(f)=\rho(\bar{f}) .
$$

By abusing notation, we will also refer to the lift of $f$ as the lift of $\bar{f}$.

Remark 3.27. Let $\left(x_{0}, \ldots, x_{q-1}\right)$ be a periodic orbit of a piecewise-smooth map whose associated circle map satisfies conditions C.1-C.4, and let $\mathbf{x}=$ $\left(\mathbf{x}_{0} \ldots \mathbf{x}_{q-1}\right) \in\{\mathcal{L}, \mathcal{R}\}^{q}$ be its associated symbolic sequence regarding the symbolic encoding given in 2.3 :

$$
\mathbf{x}_{i}=a\left(f^{i}\left(x_{i}\right)\right), 0 \leq i \leq q-1 .
$$

Then, recalling Remark 3.13 and the fact that the image of $x$ by the lift of $f$, $F(x)$, crosses an integer number $m$ when $x<c+m<F(x)$, the rotation number of $f, \rho(f)$, becomes the $\eta$ number defined in (2.6). That is, it becomes the ratio of the number of symbols $\mathcal{R}$ contained in $\mathbf{x}$ to the length of the sequence $\mathbf{x}, q$.

\subsection{Symbolic dynamics and families of orientation pre- serving maps}

In this section we will show some dynamical properties of maps satisfying conditions C.1-C.4, focusing specially on periodic orbits, their symbolic itineraries and their relation with the rotation number. In some of the results, we will additionally require the map to be contractive, however, we emphasize that, when not specified, the results that we present here do not require contractiveness. The main result in this section is the following Theorem, which is, recalling that periodic orbits of orientation preserving circle maps satisfying C.1-C.4 are well ordered, a straightforward consequence of Proposition 3.33 . 
Theorem 3.28. The symbolic sequence of the itinerary of a periodic orbit of a circle map satisfying C.1-C.4 with rotation number $P / Q$ is the one in the Farey tree of symbolic sequences associated with the rational number $P / Q$.

At the end of this section (Lemma 3.36) we show that, for a piecewise-smooth map (2.1) satisfying $i$ ) of Theorem 2.13, the $\eta$-number defined in Definition 2.11 follows a devil's staircase. This is a consequence of Theorem 3.35 proved in 34.

To show this, we recall that the set $W_{p, q}$ consists of the $q$-periodic symbolic sequences with $p$ symbols $\mathcal{R}$ (see Definition 2.9). Of special interest will be the well ordered symbolic sequences contained in these sets:

Definition 3.29. Let $\mathbf{x} \in W_{p, q}$ be a periodic symbolic sequence. Consider the (lexicographically) ordered sequence given by the iterates of $\mathbf{x}$ by $\sigma$

$$
\sigma^{i_{0}}(\mathbf{x})<\sigma^{i_{1}}(\mathbf{x})<\sigma^{i_{2}}(\mathbf{x})<\cdots<\sigma^{i_{q-1}}(\mathbf{x}) .
$$

We say that the sequence $\mathbf{x}$ is a p, q-ordered (symbolic) sequence if

$$
i_{j}-i_{j-1}=\text { constant }
$$

In other words, $\sigma$ acts on the sequence (3.11) as a cyclic permutation: there exists some $k \in \mathbb{N}, 0<k<q$, such that

$$
i_{j}=i_{j-1}+k \quad(\bmod q) .
$$

The next example illustrates the previous definition.

Example 3.30. The sequence $\mathbf{x}=\left(\mathcal{L}^{2} \mathcal{R} \mathcal{L} \mathcal{R}\right)^{\infty} \in W_{2,5}$ is 2,5 -ordered, and the sequence $\mathbf{y}=\left(\mathcal{L}^{3} \mathcal{R}^{2}\right)^{\infty} \in W_{2,5}$ is not. If we consider the four iterates of $\mathbf{x}$ and $\mathbf{y}$ by $\sigma$ we obtain

$$
\begin{aligned}
\sigma(\mathbf{x}) & =(\mathcal{L} \mathcal{R} \mathcal{L} \mathcal{R} \mathcal{L})^{\infty} & \sigma(\mathbf{y}) & =\left(\mathcal{L}^{2} \mathcal{R}^{2} \mathcal{L}\right)^{\infty} \\
\sigma^{2}(\mathbf{x}) & =\left(\mathcal{R} \mathcal{L} \mathcal{R} \mathcal{L}^{2}\right)^{\infty} & \sigma^{2}(\mathbf{y}) & =\left(\mathcal{L R}^{2} \mathcal{L}^{2}\right)^{\infty} \\
\sigma^{3}(\mathbf{x}) & =\left(\mathcal{L} \mathcal{R} \mathcal{L}^{2} \mathcal{R}\right)^{\infty} & \sigma^{3}(\mathbf{y}) & =\left(\mathcal{R}^{2} \mathcal{L}^{3}\right)^{\infty} \\
\sigma^{4}(\mathbf{x}) & =\left(\mathcal{R} \mathcal{L}^{2} \mathcal{R} \mathcal{L}\right)^{\infty} & \sigma^{4}(\mathbf{y}) & =\left(\mathcal{R} \mathcal{L}^{3} \mathcal{R}\right)^{\infty}
\end{aligned}
$$

Note that $\sigma^{5}\left(\mathbf{x}_{i}\right)=\mathbf{x}_{i}$. When we order the iterates by $\sigma$ we obtain

$$
\begin{aligned}
& \mathbf{x}<\sigma^{3}(\mathbf{x})<\sigma(\mathbf{x})<\sigma^{4}(\mathbf{x})<\sigma^{2}(\mathbf{x}) \\
& \mathbf{y}<\sigma(\mathbf{y})<\sigma^{2}(\mathbf{y})<\sigma^{4}(\mathbf{y})<\sigma^{3}(\mathbf{y})
\end{aligned}
$$

and $\mathbf{x}$ is 2,5-ordered with $k=3$ while $\mathbf{y}$ is not well ordered.

The following result identifies the symbolic sequences of periodic orbits whose lifted cycles are twist (see Definition 3.23).

Proposition 3.31 (59] Proposition III.1.1-2). Under the conditions of Proposition 3.24, if the lifted cycle $S$ is $p$, q-ordered by $F$ (see Definition 3.23) then the itinerary $I_{f}\left(x_{i}\right) \in W_{p, q}$ is a p,q-ordered symbolic sequence (see Definition 3.29). 
Proof. Let $k$ be such that

$$
(k-1) p<q \leq k p .
$$

We first note that, for $0 \leq i, j \leq q-1$ we have

$$
I_{f}\left(x_{i}\right) \leq I_{f}\left(x_{j}\right) \Longleftrightarrow i \leq j .
$$

We then write

$$
k p=q+r, r \geq 0 .
$$

Then, the result comes from the fact that

$$
x_{0}<x_{r} \leq x_{p},
$$

which occurs iff $0<r \leq p$. Assume that $r>p$. Then $q+r-p>q$ and hence $(k-1) p>q$, which contradicts 3.12 . Letting $\mathbf{x}=I_{f}\left(x_{0}\right)$, this implies

$$
\mathbf{x}<\sigma^{k} \quad{ }^{(q)}(\mathbf{x})<\sigma^{2 k} \quad{ }^{(q)}(\mathbf{x})<\cdots<\sigma^{(N-1) k} \quad{ }^{(q)}(\mathbf{x})=\sigma^{N k} \quad{ }^{(q)}(\mathbf{x}),
$$

where $N$ is the smallest such that $N k=0(\bmod q)$.

The next result formalizes what was stated in Remark 3.27 the $\eta$-number associated with the symbolic sequence of a periodic orbit (Definition 2.11) of a piecewise-smooth map satisfying i) of Theorem 2.13 becomes the rotation number (Definition 3.12) of the orientation preserving circle map obtained after the change (3.1).

Corollary 3.32. Let $f$ be an orientation preserving map, and let $x$ belong to a $q$-periodic orbit with symbolic sequence $I_{f}(x)=\mathrm{x}^{\infty} \in W_{p, q}$. Then, the rotation number becomes

$$
\rho(f)=\frac{p}{q}=\eta(\mathbf{x}) .
$$

That is, it is given by the ratio between the number of $\mathcal{R}$ symbols contained in $\mathbf{x}$ and the period, $q$, of the sequence.

Our next step consists of showing that the symbolic sequence associated with a periodic orbit of an orientation preserving circle map belongs to the Farey tree of symbolic sequences shown in Figure $3.5(\mathrm{~b})$. More precisely, we show that such a symbolic sequence is obtained by the concatenation of the symbolic sequences associated with the periodic orbits of the Farey parents of its rotation number. As a consequence of that, one obtains Theorem 3.28, announced above.

Note that this result provides an alternative isomorphism between the Farey tree of rational numbers and the Farey tree of symbolics sequences (Figures 3.5(a) and $3.5(\mathrm{~b})$, respectively) by means of the dynamical properties of circle maps.

In Section 6.4 we will present an alternative approach using the maximin properties of these sequences. 
Proposition 3.33. Let $f$ be a circle map satisfying C.1-C.4, and assume it has a periodic orbit with rotation number $P / Q,(P, Q)=1$. Let $\Delta^{\infty} \in W_{P, Q}$ be its symbolic sequence, and assume that $\Delta$ is minimal:

$$
\Delta=\min _{k \geq 0} \sigma^{k}(\Delta)
$$

Let $p, q, p^{\prime}$ and $q^{\prime}$ natural numbers such that

- $P / Q=\left(p+p^{\prime}\right) /\left(q+q^{\prime}\right)$

- $(p, q)=\left(p^{\prime}, q^{\prime}\right)=1$

- $p^{\prime} q-p q^{\prime}=1$,

that is, $p / q<p^{\prime} / q^{\prime}$ are the Farey parents of $P / Q$.

Let $\alpha^{\infty} \in W_{p, q}$ and $\beta^{\infty} \in W_{p^{\prime}, q^{\prime}}$ be the symbolic sequences of the periodic orbits with rotation numbers $p / q$ and $p^{\prime} / q^{\prime}$, respectively. Assume that $\alpha$ and $\beta$ are minimal. Then $\Delta$ is the concatenation of $\alpha$ and $\beta$ :

$$
\Delta=\alpha \beta .
$$

Proof. Let

$$
0<z_{0}<z_{1}<\cdots<z_{Q-1}<1
$$

be a periodic orbit with rotation number $P / Q$. Let us consider the lifted cycle given by $\Pi^{-1}\left(z_{i}\right)$ given in Equation 3.7 ,

$$
\begin{gathered}
0<z_{0}<z_{1}<\cdots<z_{Q-1}<1<z_{Q}<z_{Q+1}<\cdots<z_{2 Q-1}<2<z_{2 Q}<\cdots \\
\cdots<z_{P Q-1}<P<z_{P Q}<\cdots
\end{gathered}
$$

Consequently the following identity holds:

$$
z_{j+m Q}=z_{j}+m, \text { where } j, m \in \mathbb{Z} .
$$

Let $F$ be the lift of $f$ as in Definition 3.7. Due to Proposition 3.24, the points $z_{i}$ are $P, Q$-ordered by $F$ :

$$
\begin{gathered}
F\left(z_{i}\right)=z_{i+P} \\
F^{Q}\left(z_{i}\right)=z_{i}+P .
\end{gathered}
$$

We now construct two subsequences, $\left(x_{i}\right)$ and $\left(y_{i}\right)$, as follows (see Example 3.34).

The former will consist of the lifting of the set $\left\{z_{0}, f\left(z_{0}\right), \ldots, f^{q-1}\left(z_{0}\right)\right\}$ (the first $q$ iterates of $z_{0}$ ), given by

$$
\left\{z_{n P+i Q} \mid 0 \leq n \leq q-1, i \in \mathbb{Z}\right\} .
$$

Since $p$ and $q$ are relatively prime, every integer $j$ is uniquely represented as $j=n p+i q$. Let $\left(x_{j}\right)_{j \in \mathbb{Z}}$, be the sequence defined by

$$
x_{n p+i q}=z_{n P+i Q}, 0 \leq n \leq q-1, i \in \mathbb{Z} .
$$


We first prove that $\left(x_{j}\right)_{j \in \mathbb{Z}}$ satisfies

$$
\begin{gathered}
0<x_{0}<x_{1}<\cdots<x_{q-1}<1<x_{q}<x_{q+1}<\cdots<x_{2 q-1}<2<x_{2 q}<\cdots \\
\cdots<x_{p q-1}<p<x_{p q}<\cdots
\end{gathered}
$$

To see that, we show that the sequences $\left(z_{k P}\right)_{k \in \mathbb{Z}}$ and $\left(x_{k p}\right)_{k \in \mathbb{Z}}$ skip integer numbers at the same iterates; that is, if

$$
z_{(k-1) P}<i<z_{k P}(\Longleftrightarrow(k-1) P<i Q<k P),
$$

then

$$
x_{(k-1) p}<i<x_{k p}(\Longleftrightarrow(k-1) p<i q<k p) .
$$

This comes from the fact that, recalling ii) of Theorem 3.2, $p / q$ and $P / Q$ are Farey neighbours, and hence they are in the path of the Farey neighbours $i / k$ and $i /(k-1)$. Therefore we have

$$
\frac{i}{k}<\frac{p}{q}<\frac{P}{Q}<\frac{p^{\prime}}{q^{\prime}}<\frac{i}{k-1},
$$

because $P / Q$ is the Farey median of $p / q$ and $p^{\prime} / q^{\prime}$.

Let now $l=k P(\bmod Q)$ and let $t$ be such that

$$
z_{(t-1) P} \leq z_{l}<z_{t P}
$$

Then we must have

$$
x_{(t-1) p} \leq x_{\tilde{l}}<x_{t p}
$$

where $\tilde{l}=k p(\bmod q)$. Otherwise, the sequences $z_{i P}$ and $x_{i p}$ could not skip integer numbers at the same time. This shows that necessary the sequence $\left(x_{j}\right)_{j \in \mathbb{Z}}$ must satisfy (3.15). Further, it follows from (3.13) and 3.14 that

$$
x_{j+q p}=x_{j}+p, j \in(0, \ldots, q-1\} .
$$

Moreover, for $j=n p+i q, n \neq q-1$, we have

$$
F\left(x_{j}\right)=F\left(z_{n P+i Q}\right)=z_{n P+i Q+P}=x_{(n+1) p+i q}=x_{j+p} .
$$

Note that, for $n=q-1$, Equation (3.17) does not hold, as $F\left(z_{(q-1) P+i Q}\right)=$ $z_{q P+i Q}$ does not belong to the sequence $\left(x_{i}\right)_{i \in \mathbb{Z}}$.

Hence, we have that

$$
F\left(x_{j}\right)=x_{j+p}, j=n p+i q, n \neq q-1 \quad(\bmod q) .
$$

As the lifted sequence $\left(x_{j}\right)_{j \in \mathbb{Z}}$ satisfies $(3.16)$ and 3.18 , its symbolic sequence, $\alpha \in W_{p, q}$, is the one associated with a $p, q$-ordered lifted cycle.

We now obtain another subsequence derived from the last $q^{\prime}$ points of the first $Q$ iterates of the point $z_{0}$. The first point of these $q^{\prime}$ points is

$$
F^{q}\left(z_{0}\right)=z_{q P}
$$


Note that, as $p / q<p^{\prime} / q^{\prime}$ are Farey neighbours, we get

$$
1=p^{\prime} q-p q^{\prime}=p^{\prime} q+p q-p q-p q^{\prime}=\left(p^{\prime}+p\right) q-p\left(q+q^{\prime}\right)=q P-p Q .
$$

Therefore,

$$
q P=1+p Q=1 \quad(\bmod Q) .
$$

This implies that, when projecting the point $z_{q P}$ at the circle $\mathbb{R} / \mathbb{Z}$ (identified with $[0,1])$, we obtain $\Pi\left(z_{q P}\right)=z_{1}$. Therefore, when lifting the projection of the last $q^{\prime}$ points of the first $Q$ iterates of $z_{0}$, we obtain the sequence given by

$$
y_{n p^{\prime}+i q^{\prime}}=z_{1+n P+i Q}, 0 \leq n \leq q^{\prime}-1, i \in \mathbb{Z} .
$$

Arguing as before, this sequence has the form

$$
\begin{gathered}
0<y_{0}<y_{1}<\cdots<y_{q^{\prime}-1}<1<y_{q^{\prime}}<y_{q^{\prime}+1}<\cdots<y_{2 q^{\prime}-1}<2<y_{2 q^{\prime}}<\cdots \\
\cdots<y_{p^{\prime} q^{\prime}-1}<p^{\prime}<y_{p^{\prime} q^{\prime}}<\cdots
\end{gathered}
$$

and sastisfies

$$
\begin{aligned}
& F^{i}\left(y_{0}\right)=y_{i p^{\prime}}, q \leq i \leq q+q^{\prime}-1 \\
& y_{i+q^{\prime} p^{\prime}}=y_{i}+p^{\prime} .
\end{aligned}
$$

Hence, its symbolic sequence, $\beta \in W_{p^{\prime}, q^{\prime}}$ is the one associated with $p^{\prime}, q^{\prime}$-ordered lifted cycle.

Note that, $\alpha$ and $\beta$ are minimal, as they are associated with the iterates of the lowest positive point of the sequences $\left(x_{i}\right)$ and $\left(y_{i}\right), x_{0}=z_{0}$ and $y_{0}=z_{1}$, respectively.

By construction, the symbolic sequence $\Delta \in W_{P, Q}$ is the concatenation of $\alpha$ and $\beta$, as we wanted to show.

Example 3.34. Let $P / Q=8 / 11$, and assume that a circle map $f$ satisfying C.1-C.4 has rotation number $P / Q$. From Propositions 3.14 and 3.31 we know that $f$ has an 11-periodic orbit whose lifted cycle is 8,11 -ordered. We wish to show that, as given by Proposition 3.33. its symbolic sequence is given by the concatenation of the symbolic sequences associated with $p, q$ and $p^{\prime}, q^{\prime}$-ordered lifted cycles of periodic orbits with rotation number $p / q$ and $p^{\prime} / q^{\prime}$, respectively, where $p / q<p^{\prime} / q^{\prime}$ are the Farey parents of 8/11.

In order to find the Farey parents of 8/11 one can construct the Farey tree and locate them as the unique Farey neighbours of 8/11 at the Farey sequence $\mathcal{F}_{11}$. However, the proof of Proposition 3.33 gives us a method to find $q$ and $q^{\prime}$ : $q$ is the smallest such that $\Pi\left(F^{q}\left(z_{0}\right)\right)=z_{1}$.

In Figure 3.10 we show the 8,11-ordered lifted cycle, $\left(z_{i}\right)$ of the periodic orbit with rotation number $8 / 11$. Starting at $z_{0}$, the arrows provide the sequence obtained when iterating $z_{0}$ by $F$. Note that, at each iterate, one adds 8 to the subindex. We obtain that $F^{7}\left(z_{0}\right)=z_{56}$, and $56=1(\bmod 11)$. Hence, $q=7$ 


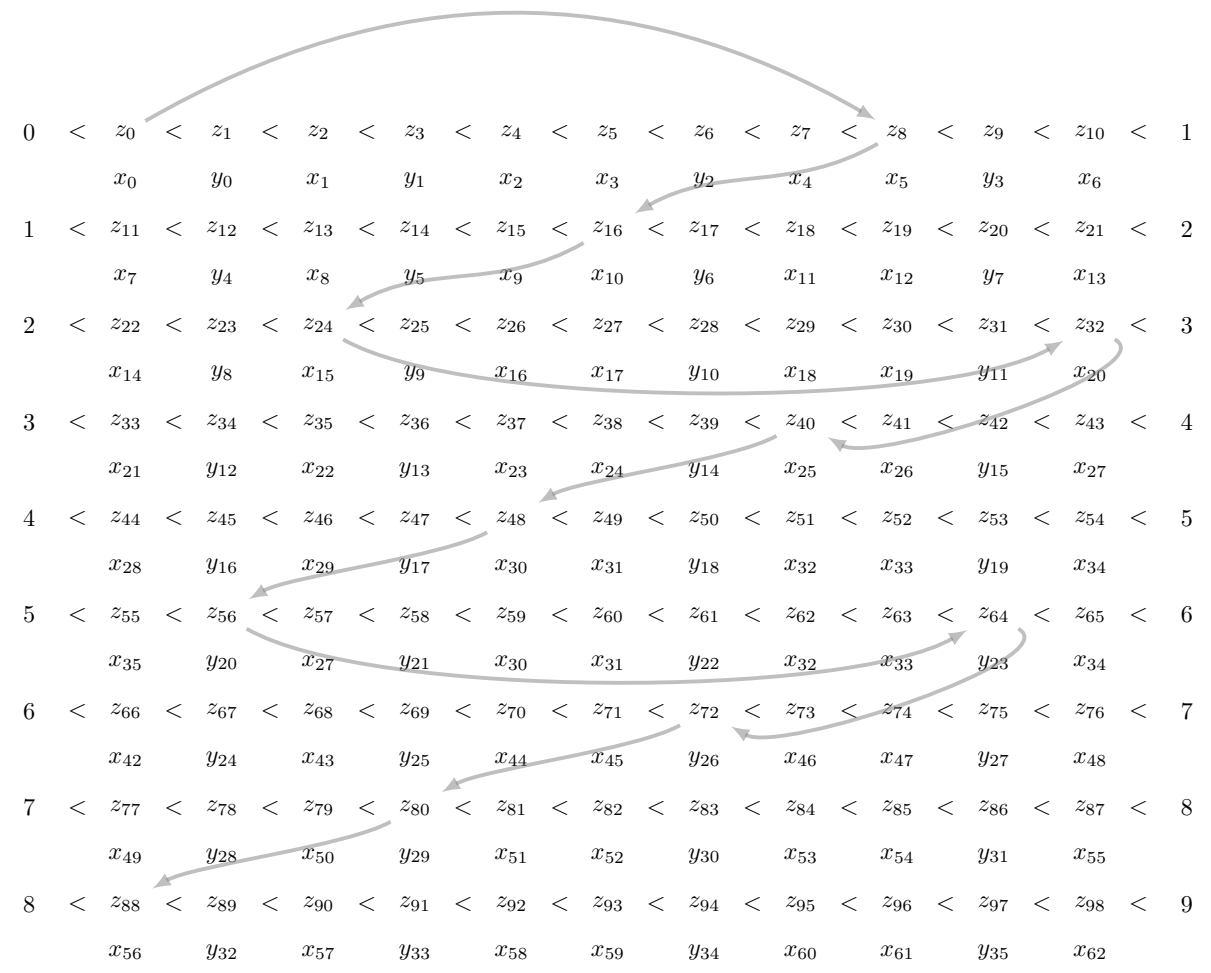

Figure 3.10: Lifted cycle of a 8 -11-periodic orbit $\left(z_{i}\right)$ split into two subsequences, $x_{i}$ and $y_{i}$, as in the proof of Proposition 3.33 (see Example 3.34 for text). 
and $q^{\prime}=11-7=4$. By using that $8=p+p^{\prime}$ and $p^{\prime} q-p q^{\prime}=1$, we obtain $p=5$ and $p^{\prime}=3$ :

$$
\frac{8}{11}=\frac{5+3}{7+4}
$$

We can now construct the sequence $x_{i}$. We start with $x_{0}=z_{0}$ and we add 5 to the subindex of $x$ at each iteration: $x_{5 i}=z_{8 i}$, for $0 \leq i<7$. This provides the points $x_{0}<x_{5}<x_{10}<\cdots<x_{30}$. By lifting these points (adding multiples of 7 to their subindex's), we obtain a lifted cycle defined in Equation (3.14), which is 5,3-ordered.

Next we construct the subsequence $y_{i}$. Following Equation (3.19), we start with $y_{p q^{\prime}}=y_{20}=z_{1+p Q}=z_{56}$. By further iterating, we add $p^{\prime}$ at the subindex's of $y_{i}: y_{20+i p^{\prime}}=z_{56+i P}$, for $0 \leq i<4$. This leads to the sequence $y_{20}<y_{23}<$ $\cdots<y_{29}$. Finally, by lifting these points, that is, adding multiples of 4 to their subindexes, we obtain the 3,4-ordered lifted cycle of a periodic orbit with rotation number $3 / 4$.

Note that the next iterate of $y_{29}=z_{80}$ becomes $z_{88}$, which satisfies $\Pi\left(z_{80}\right)=$ $z_{0}$. Hence, after following the sequence $\left(y_{i}\right)$, the lifted cycle $\left(z_{i}\right)$ switches back to the sequence $\left(x_{i}\right)$, and the symbolic sequence is repeated.

In the rest of this section we study the rotation number for families of orientation preserving circle maps; that is, under the variation of the parameter $c$ in condition $C .3$, which, by means of the change of variables given in Equation (3.1), is equivalent to the parameter $\lambda$ of parametrizing the curve in parameter space mentioned in Theorem 2.13 .

Recalling Proposition 3.21 and Lemma 3.22 we already have that, when varying $\lambda$ from 0 to 1 , the rotation number (and hence the $\eta$-number) is continuous and monotonically increases from 0 to 1 . In order to show that, moreover, it is a devil's staircase, we need to show that, in addition, it is constant for all values of $\lambda$ except for a Cantor set of zero measure. This is will come from the following

Theorem 3.35 ([34], Theorem 1'). Let $f$ be a continuous monotonic nondecreasing map of the circle of degree one satisfying

i) $f$ is constant on an interval $[a, b]$ and of class $C^{1}$ outside $[a, b]$

ii) $\inf _{y \notin[a, b]}\left(f^{\prime}(y)\right)>1$

Let $f_{t}$ be the map defined by $f_{t}(y)=f(y)+t, t \in[0,1]$. Let

$$
E=\left\{t \mid f_{t} \text { has irrational rotation number }\right\} \text {. }
$$

Then $m(E)=0$, where $m$ denotes Lebesgue measure, and furthermore $E$ has zero Hausdorff dimension.

Generalizations of the previous theorem can be found in [131, 121.

Finally, we show that Theorem 3.35 extends to a piecewise-smooth system of the form 2.1 satisfying $h .1-h .3$ and $i$ ) of Theorem 2.13 (or an orientation 


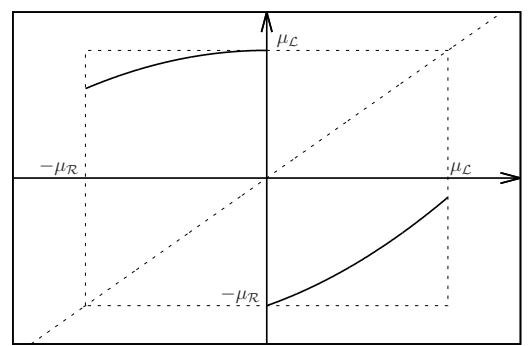

(a)

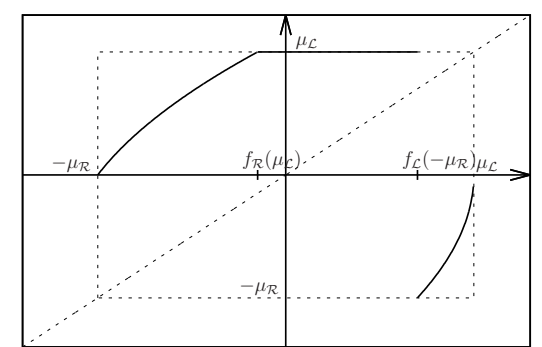

(b)

Figure 3.11: (a) piecewise-smooth map satisfying C.1-C.4. (b) its inverse.

preserving circle map satisfying C.1-C.4). This will prove that the $\eta$-number follows a devil's staircase.

Lemma 3.36. Let $f$ be piecewise-smooth map as in Equation (2.1) satisfying conditions h.1-h.3 and i) of Theorem 2.13. and let $\bar{f}_{\lambda}$ be the orientation preserving circle map obtained after applying the change of variables (3.1). Then, the set of values of $\lambda$ for which the map $\bar{f}_{\lambda}$ has irrational rotation number consists of a Cantor set with zero measure.

Proof. We note that the map $\bar{f}_{\lambda}$ is invertible. Let $\phi$ be as in (3.1) and define

$$
\begin{aligned}
a & :=\bar{f}\left(1^{-}\right)=\phi\left(f_{\mathcal{R}}\left(\mu_{\mathcal{L}}\right)\right) \\
b & :=\bar{f}\left(0^{+}\right)=\phi\left(f_{\mathcal{L}}\left(-\mu_{\mathcal{R}}\right)\right) .
\end{aligned}
$$

Then, the inverse $\bar{f}_{\lambda}^{-1}(y)$ is an increasing expanding map with a "hole" for $y \in[a, b]$ (see Figure 3.11(b)]. As the trajectories of any point $x \in[0,1]$ by $\bar{f}$ do not reach the interval $[a, b]$, we can proceed as the proof of Proposition 3.25 and complete the map $\bar{f}_{\lambda}^{-1}$ with a horizontal part equal to 1 for $y \in[a, b]$. This allows us to consider a map

$$
g(y)= \begin{cases}\phi^{-1} \circ f_{\mathcal{R}}^{-1} \circ \phi(y) & \text { if } y \in[0, a] \\ 1 & \text { if } y \in[a, b] \\ \phi^{-1} \circ f_{\mathcal{L}}^{-1} \circ \phi(y) & \text { if } y \in[b, 1]\end{cases}
$$

which coincides with $\bar{f}_{\lambda}^{-1}(y)$ for $y \notin(a, b)$. Let

$$
\begin{array}{ccc}
\gamma:[0,1] & \longrightarrow & \mathbb{R}^{2} \\
\lambda & \longmapsto & \left(\mu_{\mathcal{L}}(\lambda), \mu_{\mathcal{R}}(\lambda)\right)
\end{array}
$$




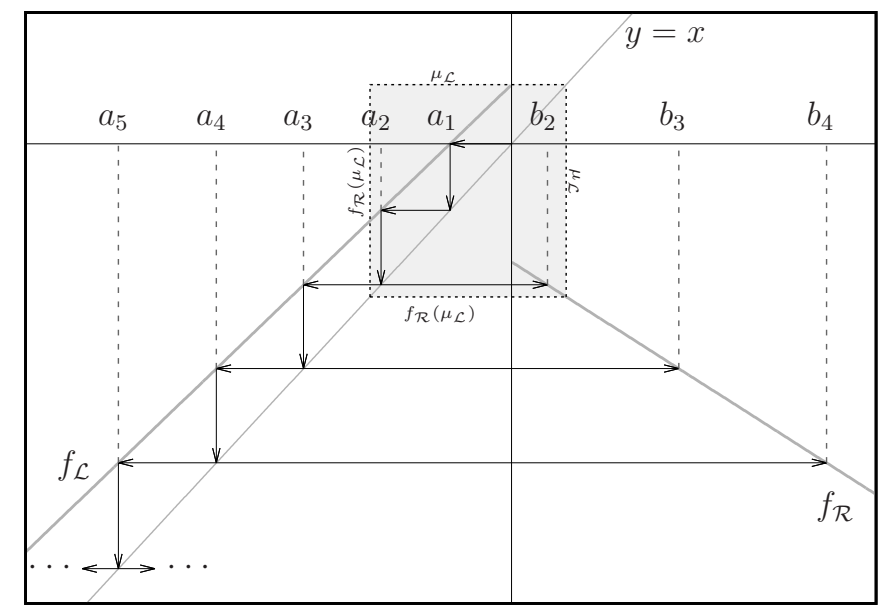

Figure 4.12: Piecewise-smooth map as in Equation (2.1) satisfying ii) of Theorem 2.13 and the sequences defined in Equation (4.2)-(4.3) The gray box represents the absorbing interval 4.1.

be a parameterization satisfying $H .1-H .3$ of Theorem 2.13 . Then, the interval $[a, b]$ smoothly varies from $\left[0, \phi\left(f_{\mathcal{L}}\left(-\mu_{R}(0)\right)\right)\right]$ to $\left[\phi\left(f_{\mathcal{R}}\left(\mu_{\mathcal{L}}(1)\right)\right), 1\right]$ when $\lambda$ is varied from $\lambda=0$ to $\lambda=1$. Let $g_{\lambda}(y)$ be $g(y)$ after applying the reparameterization $\gamma$. Then, we have

$$
g_{\lambda}(y)=g_{0}(y)+\lambda,
$$

and hence we can apply Theorem 3.35 and get the result.

\section{Non-orientable case}

The non-orientable (increasing-decreasing) case occurs under conditions ii) of Theorem 2.13. In this case, the map 2.1 becomes increasing for $x<0$ and decreasing for $x>0$.

As mentioned in Section 1 and Section 2.3 this situation was discussed in 80 Section 3.3 and proven in full detail in [18]. For completeness, we provide in this section an overview of this proof.

We first observe (see Figure 4.12 that a map $f$ as in Equation 2.1) satisfying conditions $h .1-h .3$ and $i$ ) of Theorem 2.13 is a map on the interval

$$
f:\left[f_{\mathcal{R}}\left(\mu_{\mathcal{L}}\right), \mu_{\mathcal{L}}\right] \longrightarrow\left[f_{\mathcal{R}}\left(\mu_{\mathcal{L}}\right), \mu_{\mathcal{L}}\right]
$$




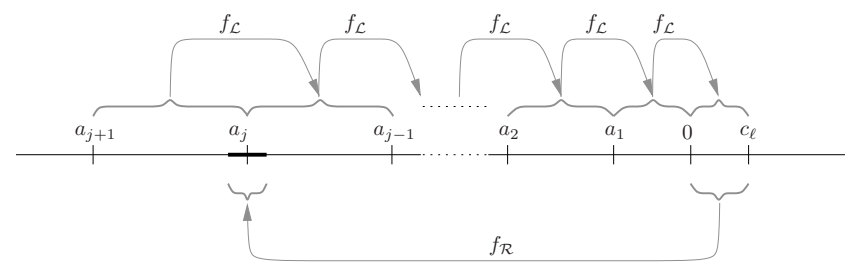

Figure 4.13: Backward and forward iterates of $\left(0, \mu_{\mathcal{L}}\right] . f_{\mathcal{R}}\left(\left(0, \mu_{\mathcal{L}}\right]\right)$ (dark segment) is smaller than $f_{\mathcal{L}}^{-n}\left(\left(0, \mu_{\mathcal{L}}\right]\right) \forall n$. Therefore, at most one $a_{j}$ can be reached by $f_{\mathcal{R}}\left(\left(0, \mu_{\mathcal{L}}\right]\right)$.

We now consider the symbolic itineraries of periodic orbits for a map 4.1 under the mentioned conditions. Clearly, when the parameters $\mu_{\mathcal{L}}$ and $\mu_{\mathcal{R}}$ are varied along the parametrization (2.7) satisfying H.1-H.3, the map 4.1) becomes negative for $x \in\left[0, \mu_{\mathcal{L}}\right]$. Hence, periodic orbits cannot have symbolic itineraries with consecutive $\mathcal{R}$ symbols.

In order to show that only possible itineraries for periodic orbits are of the form $\mathcal{L}^{n} \mathcal{R}$, we define the sequences of preimages of $x=0$ by $f_{\mathcal{L}}$ and $f_{\mathcal{R}}$ :

$$
\begin{aligned}
& a_{0}=0, \quad a_{n}=f_{\mathcal{L}}^{-1}\left(a_{n-1}\right) \quad \text { if } n>0, \\
& b_{n}=f_{\mathcal{R}}^{-1}\left(a_{n}\right) \quad \text { if } n \geq n_{0} .
\end{aligned}
$$

Due to the contractiveness of $f_{\mathcal{R}}$ and $f_{\mathcal{L}}$, we have that

$$
\begin{gathered}
\frac{m\left(\left[a_{n+1}, a_{n}\right]\right)}{m\left(\left[a_{n}, a_{n-1}\right]\right)}>1, n>0 \\
\frac{m\left(\left[b_{n}, b_{n+1}\right]\right)}{m\left(\left[b_{n-1}, b_{n}\right]\right)}>1, n>n_{0},
\end{gathered}
$$

where $m$ indicates the length of the interval.

Then we have the following

Lemma 4.1. Suppose $f$ is a map of type 2.1) fulfilling conditions h.1-h.3 and ii) of Theorem 2.13. Then there exists at most one $a_{j}$ (equiv. $b_{j}$ ) such that $a_{j} \in f_{r}\left(\left(0, \mu_{\mathcal{L}}\right]\right)$ (equiv. $\left.b_{j} \in\left(0, \mu_{\mathcal{L}}\right]\right)$.

Proof. Recalling that $\mu_{\mathcal{L}}=f_{\mathcal{L}}(0)$, one has (see Figure 4.13)

$$
\begin{aligned}
{\left[a_{n+1}, a_{n}\right] } & =f_{\mathcal{L}}^{-1}\left(\left[a_{n}, a_{n-1}\right]\right) \\
{\left[a_{1}, 0\right] } & =f_{\mathcal{L}}^{-1}\left(\left[0, \mu_{\mathcal{L}}\right]\right) .
\end{aligned}
$$

Using the property shown in Equation (4.4) one has

$$
m\left(\left[0, c_{m}\right]\right)<m\left(\left[a_{n+1}, a_{n}\right]\right) \forall n,
$$

and, since $f_{\mathcal{R}}$ is a contracting one obtains

$$
m\left(f_{r}\left(\left(0, \mu_{\mathcal{L}}\right]\right)<m\left(\left[a_{n+1}, a_{n}\right]\right) \forall n .\right.
$$

Therefore, at most one $a_{n}$ can be located in $f\left(\left(0, \mu_{\mathcal{L}}\right]\right)$. 
The next Lemma tells us what symbolic itineraries are possible.

Lemma 4.2 (18 Lemma 7). Let $f$ be a piecewise-smooth map as considered above, and let $x_{0}<\cdots<x_{n}, x_{i} \in\left[f_{\mathcal{R}}\left(\mu_{\mathcal{L}}\right), \mu_{\mathcal{L}}\right]$ be a periodic orbit. Then, $I_{f}\left(x_{0}\right)=\left(\mathcal{L}^{n} \mathcal{R}\right)^{\infty}$.

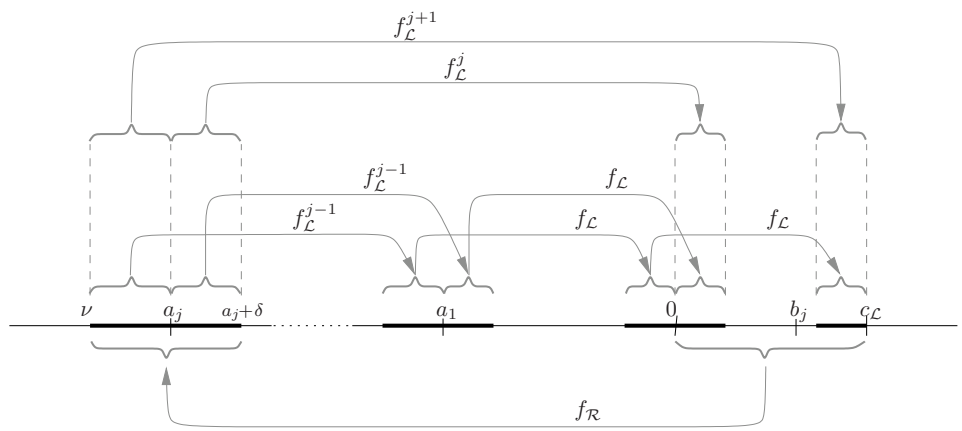

Figure 4.14: The interval $f_{\mathcal{R}}\left(\left(0, \mu_{\mathcal{L}}\right]\right)$ is split when it returns to the right domain.

The proof of this Lemma is in fact an extension of the arguments presented in [80. Section 3.3, and was provided in full detail in [18. We provide it here for completeness.

Proof. If $f$ has periodic orbit of period $n \geq 2$, we necessary have

$$
f_{\mathcal{R}}\left(\left(0, \mu_{\mathcal{L}}\right]\right) \cap\left[a_{n}, a_{n-1}\right] \neq \emptyset,
$$

which can be given due to one of the next three situations (see Figures 4.13 and 4.14

S.1 $a_{n-1} \in f_{\mathcal{R}}\left(\left(0, \mu_{\mathcal{L}}\right]\right)$

$\mathrm{S} .2 f_{\mathcal{R}}\left(\left(0, \mu_{\mathcal{L}}\right]\right) \subset\left(a_{n}, a_{n-1}\right)$

S.3 $a_{n} \in f_{\mathcal{R}}\left(\left(0, \mu_{\mathcal{L}}\right]\right)$

If S.1 holds, $b_{n-1} \in\left(0, \mu_{\mathcal{L}}\right]$ and

$$
\begin{aligned}
f_{\mathcal{L}}^{n} f_{\mathcal{R}}:\left[b_{n-1}, \mu_{\mathcal{L}}\right] & \longrightarrow\left[b_{n-1}, \mu_{\mathcal{L}}\right] \\
f_{\mathcal{L}}^{n-1} f_{\mathcal{R}}:\left(0, b_{n-1}\right) & \longrightarrow\left(0, b_{n-1}\right),
\end{aligned}
$$

are continuous contracting functions which must have a unique (stable) fixed point. Therefore, two stable periodic orbits of type $\mathcal{L}^{n} \mathcal{R}$ and $\mathcal{L}^{n-1} \mathcal{R}$ coexist. Note that for $n=2$ this proves also the existence of a $\mathcal{L} \mathcal{R}$-periodic orbit.

In the second case $(\mathrm{S} .2), b_{n-1} \notin\left(0, \mu_{\mathcal{L}}\right]\left(\left[0, \mu_{\mathcal{L}}\right] \subset\left(b_{n-1}, b_{n}\right)\right)$ and

$$
f_{\mathcal{L}}^{n} f_{\mathcal{R}}:\left(0, \mu_{\mathcal{L}}\right] \longrightarrow\left(0, \mu_{\mathcal{L}}\right]
$$


is a continuous contracting function which also must have a unique (stable) fixed point. In this case, there exists a unique periodic orbit of type $\mathcal{L}^{n} \mathcal{R}$ which is the unique attractor in $\left(0, \mu_{\mathcal{L}}\right]$.

Finally, if S.3 holds, replacing $n$ by $n-1$ and arguing as in S.1, one has that a stable periodic orbit of type $\mathcal{L}^{n} \mathcal{R}$ coexists with a stable $\mathcal{L}^{n+1} \mathcal{R}$-periodic one.

Remark 4.3. By contrast to all periodic orbits of type $\mathcal{L}^{n} \mathcal{R}$ with $n \geq 2$, the periodic orbit $\mathcal{L} \mathcal{R}$ exists not only for $\mu_{\mathcal{R}}>0$ but also for $\mu_{\mathcal{R}} \leq 0$. In that case, it coexists with the fixed point $\mathcal{R}\left(\mathcal{L}^{0} \mathcal{R}\right)$ (see Figure 2.3).

Remark 4.4. Note that the transitions between cases S.1, S.2 and S.3 are given by border collision bifurcations where the respective periodic orbits are created or destroyed when they collide with the boundary $x=0$. This defines the border collision bifurcation curves shown in Figure 2.3.

Remark 4.5. As it is known, invariant objects of piecewise-smooth systems do not necessarily have to be separated by another invariant object. In this case, the coexistence of stable periodic objects may also be separated by the discontinuity (and its preimages).

We focus finally on the bifurcation scenario shown inf Figures 2.32 .4 when the parameter $\lambda$ of the parametrization (2.7) is varied.

Clearly, the successions $a_{n}$ and $b_{n}$ are continuous functions of the parameter $\gamma$. Hence, the transitions between S.1, S.2 and S.3 described in the proof of Lemma 4.2. That is, assume that for some $\gamma$ S.1 holds; then two periodic orbits of type $\mathcal{L}^{n} \mathcal{R}$ and $\mathcal{L}^{n-1} \mathcal{R}$ coexists. Due to conditions H.1-H.3, when decreasing $\gamma, \mu_{\mathcal{L}}$ monotonically decreases to zero, whereas $\mu_{\mathcal{R}}$ increases towards some bounded value. Hence, $a_{n-1}$ monotonically decreases to zero and, hence, as the $m\left(\left[0, \mu_{\mathcal{L}}\right]\right)<m\left(\left[a_{n}, a_{n-1}\right]\right)$, there exists some value of $\gamma$ for which $f_{\mathcal{R}}\left(\left[0, \mu_{\mathcal{L}}\right]\right) \subset\left(a_{n}, a_{n-1}\right)$ and S.2 holds. Hence, the $\mathcal{L}^{n-1} \mathcal{R}$-periodic orbit bifurcates and only a $\mathcal{L}^{n} \mathcal{R}$-periodic orbit exists. Arguing similarly, by further decreasing $\gamma$, S.3 holds, a $\mathcal{L}^{n+1} \mathcal{R}$-periodic orbit bifurcates and coexists with the $\mathcal{L}^{n} \mathcal{R}$-periodic orbit.

As this occurs for all $n$, this argument proves the bifurcation scenario described in Section 2.3 .

\section{Some remarks on piecewise-smooth expand- ing maps}

Frequently, both in applications and theoretical studies, the contracting conditions required in the previous sections become too restrictive and one needs to deal with discontinuous maps exhibiting expansivenesses. However, such maps are shown to undergo similar bifurcations as the ones described in Section 2 Examples are found in the study of homoclinic bifurcations, such as the Lorenz system $([75,120,112,68,70,82])$ or in Multiscale dynamics $(99,41])$, but also in power electronics $([76,30,33,115,102,87,136,19])$, biology [89, 106, 116] 
or economy ([127]), among others. Although the results summarized in Theorem 2.13 cannot be applied when the map lacks of contractivness, many of the results presented in Sections 3 and 4 are still valid under the assumption of weak enough expansion. In this section we review possible scenarios led by the loss of contractiveness, that is, we focus on maps as in Equation (2.1) satisfying

$h^{\prime} .1 f_{\mathcal{L}}(0)=f_{\mathcal{R}}(0)=0$.

$h^{\prime} .20<\left(f_{\mathcal{L}}(x)\right)^{\prime}, x \in(-\infty, 0)$

$h^{\prime} .30<\left|\left(f_{\mathcal{R}}(x)\right)^{\prime}\right|, x \in(0, \infty)$,

with $f_{\mathcal{L}}$ and $f_{\mathcal{R}}$ may be expanding in all or part if their domains.

As before, we now distinguish between the orientable $\left(\left(f_{\mathcal{R}}(x)\right)^{\prime}>0\right)$ and non-orientable $\left(\left(f_{\mathcal{R}}(x)\right)^{\prime}<0\right)$ cases.

\subsection{Orientable case}

Let us assume that conditions $h^{\prime} .1-h^{\prime} .3$ hold with $\left(f_{\mathcal{R}}(x)\right)^{\prime}>0$. We focus on the case $\mu_{\mathcal{L}}, \mu_{\mathcal{R}}>0$. We first note that, as long as

$$
f_{\mathcal{L}}\left(-\mu_{\mathcal{R}}\right) \geq f_{\mathcal{R}}\left(\mu_{\mathcal{L}}\right)
$$

the piecewise-smooth map $f$ is still invertible. Hence, as in the contractive case, it can be reduced to a circle map of type $(3.2)$ satisfying conditions C.1-C.4 by means of the smooth change of variables (3.1). Therefore, all the results in Section 3 requiring only that the lift of the map is strictly increasing (which is given by conditions $C .1-C .4$ ) also hold as long as Equation (5.1) is fulfilled. Thats is, recalling that $\lambda$ parametrizes the curves in Equation (2.7), we get that, for any $\lambda \in[0,1]$ such that 5.1] is satisfied, we have that

i) the rotation number $(\rho)$ given in Definition 3.12 is well defined, unique and increasing as a function of $\lambda$,

ii) if $\rho=p / q$, with $p, q$ co-prime, then $f$ possesses a $p, q$-ordered periodic orbit whose symbolic sequence belongs to the Farey tree of symbolic sequences.

Note that ii) is a consequence of Proposition 3.14, which requires only conditions C.1-C.4. However, as noted in Remark 3.20, when the maps $f_{\mathcal{L}}$ and $f_{\mathcal{R}}$ exhibit expansiveness, this periodic may be not only repelling but also non unique. However, even in this case, all existing periodic orbits are $p, q$-ordered, as Proposition 3.24 still holds, and therefore they all must have the same symbolic dynamics.

Regarding the dynamics for $\rho \in \mathbb{R} \backslash \mathbb{Q}$, Proposition 3.25 does not hold if $f_{\mathcal{L}}$ or $f_{\mathcal{R}}$ are expanding. The main obstacle is given by the fact that Corollary 3.3 of 131 cannot be applied to show that $\omega(f)$ is a Cantor set (see the proof of Proposition 3.25). As a consequence, one needs additional conditions to state whether $\omega(f)$ is a Cantor set or the whole circle. 
As opposite to the purely contractive case, when $f_{\mathcal{L}}$ or $f \mathcal{R}$ are expanding, the rotation number no longer necessary follows a devil's staircase. Without the contracting assumption Theorem 3.35 does not hold and hence the set of values of $\lambda$ for which the piecewise-smooth map does not possess any periodic orbit (the rotation number of the associated circle map is irrational) does not necessary have zero measure. However, $\rho(\lambda)$ is still a non-decreasing continuous function.

As stated above, the crucial property that keeps most of the results shown in Section 3 valid even when the map is expansive is the invertibility condition (5.1). When this is lost, then the corresponding lift exhibits negative gaps and is not an increasing map. As a consequence, the rotation number becomes non-unique. Instead, when needs to deal with rotation intervals, coexistence of periodic orbits with different rotation numbers and symbolic dynamics and positive entropy. Although there exist many results in the literature (see for example [2, 4, 3, 1, 5, 6]), a precise description of the bifurcation scenarios becomes difficult under general assumptions might be difficult to state.

\subsection{Non-orientable case}

Let us now consider a class of maps as in (2.1) satisfying

$h " .1 f_{\mathcal{L}}(0)=f_{\mathcal{R}}(0)=0$.

$h " .20<\left(f_{\mathcal{L}}(x)\right)^{\prime}, x \in(-\infty, 0)$

$h " .30>\left|\left(f_{\mathcal{R}}(x)\right)^{\prime}\right|, x \in(0, \infty)$,

with $f_{\mathcal{L}}$ and $f_{\mathcal{R}}$ may be expanding in all or part if their domains.

Unlike in the orientable case, such a map fulfilling conditions $h$ ". $1-h$ ". 3 is always invertible for $\mu_{\mathcal{L}}, \mu_{\mathcal{R}}>0$. However, even in the linear case, when the map $f_{\mathcal{L}}$ or $f_{\mathcal{R}}$ is expanding, the bifurcation scenario may change significantly (see for example [15]). On one hand, provided that the existence and pairwise uniqueness of periodic orbits for the contracting case relies on Brower's fixed point Theorem, it may happen that no periodic orbits may exist or several unstable ones may co-exist. On the other hand, whenever they exist, symbolic sequences associated with periodic orbits may be very different. Obviously, provided that, for $\mu_{\mathcal{R}}>0, f_{\mathcal{R}}(x) \leq 0$ if $x \geq 0$, no symbolic sequences can contain two consecutive $\mathcal{R}$ 's. Then, symbolic sequences may contain blocks of the form $\mathcal{L}^{n} \mathcal{R} \mathcal{L}^{m}$, with $n \neq m$. Numerical studies have also shown evidence of the existence of chaotic attractors, whose associated symbolic dynamics are similar ([12, 13, 14]).

However, if $f_{\mathcal{R}}$ is expansive but $f_{\mathcal{L}}$ is not, it may happen that, for $\mu_{\mathcal{L}}>0$ smaller than a certain quantity (which may depend on $\mu_{\mathcal{R}}$ ), the number of iterations performed in the negative domain may be large enough to compensate the expanding dynamics of $f_{\mathcal{R}}$. This is because the smaller $\mu_{\mathcal{L}}>0$ the larger the number of iterations needed to return to the right domain. Hence, in such situation, one recovers the period incrementing scenario described in Section 2.3 . 
with and attracting periodic orbits of type $\mathcal{L}^{n} \mathcal{R}$ with $n$ larger than a certain value. As in the attracting case, there will exist parameter values for which there exists coexistence of periodic orbits of type $\mathcal{L}^{n} \mathcal{R}$ and $\mathcal{L}^{n+1} \mathcal{R}$.

\section{Maximin itineraries and piecewise-smooth maps in $\mathbb{R}^{n}$}

\subsection{Introduction}

Often, due to the complexity of real applications, one-dimensional maps are

not enough and one needs to consider maps in higher dimensions. Examples of two (or higher)-dimensional piecewise-smooth maps are found as Poincaré (or stroboscopic) maps in non-autonomous mechanical systems ([37, 109, 44]), but their are also found in power electronics $(45,29$, , 113, 67, 136, 7]), control theory $(46,133,135,51])$ or mathematical-neuroscience or biology $([124,125,57$, 103, 123, 116]). Piecewise-smooth maps in higher dimensions are also obtained when studying Filippov flows in $\mathbb{R}^{n}(n \geq 3)$ (49); they appear as half-return Poincaré maps, and become discontinuous close to grazing and Hopf bifurcations ([56, 108, 94, 111, 39, 38]).

As it occurs with homoclinic bifurcations for smooth flows (see Sections 1], 2.2 and 7.1.1 for references), symbolic dynamics of piecewise-smooth discontinuous maps in $\mathbb{R}^{n}$ may help to understand the number of loops performed by periodic orbits in different zones of the state space $([108,38])$. There exist some general results for piecewise-smooth continuous maps on the plane ([119, 117, 55]) (see [118] for a recent survey). Unfortunately, although some efforts have been done by exhaustively studying particular systems ( $42,69,29$, 93, 48, 54, 57, 7]) there exists very few literature providing general results for general piecewisesmooth discontinuous maps.

\subsection{Maximin/minimax properties of symbolic sequences}

We continue at the symbolic level by defining the maximin/minimax properties of symbolic sequences in $W_{p, q}$.

Definition 6.1. We say that a symbolic sequence $\mathbf{x} \in W_{p, q}$ is

- maximin if

$$
\min _{0 \leq k \leq q}\left(\sigma^{k}(\mathbf{x})\right)=\max _{\mathbf{y} \in W_{p, q}}\left(\min _{0 \leq k \leq q}\left(\sigma^{k}(\mathbf{y})\right)\right)
$$

- minimax if

$$
\max _{0 \leq k \leq q}\left(\sigma^{k}(\mathbf{x})\right)=\min _{\mathbf{y} \in W_{p, q}}\left(\max _{0 \leq k \leq q}\left(\sigma^{k}(\mathbf{y})\right)\right) .
$$

As it was proven in [32, 61] using different techniques, the maximin and minimax properties are equivalent. That is, one has the following 
Theorem $6.2([32,61])$. Let $\mathbf{x} \in W_{p, q}$. Then, $\mathbf{x}$ is maximin if and only if it is minimax.

Example 6.3. Let $\eta=2 / 5$. Up to cyclic permutations, there exist only two periodic sequences in $W_{2,5}$, which are represented by means of the minimal and maximal blocks

$$
\begin{array}{r}
\mathcal{L}^{3} \mathcal{R}^{2}=\min _{0 \leq k \leq q}\left(\sigma\left(\mathcal{L}^{3} \mathcal{R}^{2}\right)\right) \\
\mathcal{R}^{2} \mathcal{L}^{3}=\sigma^{2}\left(\mathcal{L}^{3} \mathcal{R}^{2}\right)=\max _{0 \leq k \leq q}\left(\sigma\left(\mathcal{L}^{3} \mathcal{R}^{2}\right)\right)
\end{array}
$$

and

$$
\begin{aligned}
\mathcal{L}^{2} \mathcal{R} \mathcal{L} \mathcal{R} & =\min _{0 \leq k \leq q}\left(\sigma\left(\mathcal{L}^{2} \mathcal{R} \mathcal{L} \mathcal{R}\right)\right) \\
\mathcal{R} \mathcal{L} \mathcal{R} \mathcal{L}^{2}=\sigma^{2}\left(\mathcal{L}^{2} \mathcal{R} \mathcal{L} \mathcal{R}\right) & =\max _{0 \leq k \leq q}\left(\sigma\left(\mathcal{L}^{2} \mathcal{R} \mathcal{L} \mathcal{R}\right)\right)
\end{aligned}
$$

Then, as

$$
\begin{aligned}
& \mathcal{L}^{2} \mathcal{R} \mathcal{L} \mathcal{R}>\mathcal{L}^{3} \mathcal{R}^{2} \\
& \mathcal{R} \mathcal{L} \mathcal{R} \mathcal{L}^{2}<\mathcal{R}^{2} \mathcal{L}^{3},
\end{aligned}
$$

the sequence $\mathcal{L}^{2} \mathcal{R} \mathcal{L} \mathcal{R}$ is minimax and maximin.

The following result tells us that all the sequences shown in the Farey tree of symbolic sequences (Figure 3.5(b) (given by consecutive concatenation), are maximin (minimax).

Proposition 6.4 ([59] Proposition II.2.4-3). Let $p / q<p^{\prime} / q^{\prime}$ be the irreducible form of two Farey neighbours, and let $\mathbf{x}^{\infty} \in W_{p, q}$ and $\mathbf{y}^{\infty} \in W_{p^{\prime}, q^{\prime}}$ two maximin sequences, with $\mathbf{x} \in\{\mathcal{L}, \mathcal{R}\}^{q}$ and $\mathbf{y} \in\{\mathcal{L}, \mathcal{R}\}^{q^{\prime}}$ minimal blocks. Then, the sequences given by the concatenation of these two blocks $(\mathbf{x y})^{\infty} \in W_{\left(p+p^{\prime}\right) /\left(q+q^{\prime}\right)}$ is maximin.

We provide a sketch of the proof provided in [59].

Proof. One first sees that $\mathbf{x}$ and $\mathbf{y}$ belong to the same domain of some deflation; i.e, a map which collapses blocks contained in sequences as follows:

$$
\pi=\left\{\begin{array}{l}
\mathcal{L}^{n+1} \mathcal{R} \longrightarrow \mathcal{R} \\
\mathcal{L}^{n} \mathcal{R} \longrightarrow \mathcal{L}
\end{array}\right.
$$

Then, one proceeds by induction using that $\pi(\mathbf{x})$ and $\pi(\mathbf{y})$ are maximin, and also is $\pi^{-1}(\mathbf{x y})$. See [59] for more details.

The following result tells us that maximin symbolic sequences are also well ordered sequenes (see Definition 3.29). 
Theorem 6.5 (61]). Let $\mathbf{x} \in W_{p, q}$. Then, $\mathbf{x}$ is maximin if and only if it is $p, q$-ordered.

The previous result was also proven in 32 for the one-dimensional case using endomorphisms of the circle. Moreover, note that, also for the one-dimensional case we already have such result by using the Farey tree of symbolic sequences and Proposition 3.33 However, the previous Theorem was proven using only symbolic properties.

\subsection{Quasi-contractions and piecewise-smooth maps in $\mathbb{R}^{n}$}

In this section we review some results for piecewise-smooth maps in $\mathbb{R}^{n}$ regarding their symbolic properties.

We consider maps defined in some suitable open set $U \subset \mathbb{R}^{n}$,

$$
f: U \longrightarrow U
$$

of the following form. Let

$$
h: \mathbb{R}^{n} \longrightarrow \mathbb{R}
$$

some differentiable function. We then consider a switching manifold $\Sigma \subset U$ as

$$
\Sigma=h^{-1}(0) \cap U \text {. }
$$

which splits $U$ in two subsets

$$
E_{\mathcal{L}}=\{x \in U, \mid h(x)<0\} \quad \text { and } \quad E_{\mathcal{R}}=\{x \in U, \mid h(x)>0\} .
$$

Then we write such maps as

$$
f: E_{\mathcal{L}} \cup E_{\mathcal{R}} \longrightarrow U
$$

defined as

$$
f(x)= \begin{cases}f_{\mathcal{L}}(x) & \text { if } x \in E_{\mathcal{L}} \\ f_{\mathcal{R}}(x) & \text { if } x \in E_{\mathcal{R}}\end{cases}
$$

where

$$
f_{\mathcal{L}}: \mathbb{R}^{n} \longrightarrow \mathbb{R}^{n} \quad \text { and } \quad f_{\mathcal{R}}: \mathbb{R}^{n} \longrightarrow \mathbb{R}^{n}
$$

are two smooth maps.

Note that in Equation (6.1) does not define $f$ in $\Sigma$. Similarly as we did for the one-dimensional case, we will consider that $f$ is bi-valued for $x \in \Sigma$ :

$$
f(\Sigma)=f_{\mathcal{L}}(\Sigma) \cup f_{\mathcal{R}}(\Sigma) .
$$

Given $x \in U$ one can also define its symbolic itinerary by $f$ as in Equation 2.2 . by considering now the encoding

$$
a(x)= \begin{cases}\mathcal{R} & \text { if } x \in E_{\mathcal{R}} \\ \mathcal{L} & \text { if } x \in E_{\mathcal{L}}\end{cases}
$$


Note that, if $\mathbf{x}$ is the symbolic sequence associated with a periodic orbit of a map of type (6.1) then it still makes sense to consider its $\eta$-number, $\eta(\mathbf{x})$, as defined in Equation (2.6). However, we will not provide a definition of the rotation number for such maps, although the classical definition through the lift for the one-dimensional case (see Definition 3.12 could be extended by considering $f$ as a map onto an $n$-dimensional cylinder (see Section 8 for a discussion).

Below we will assume now that $f$ is a contraction (or just $f$ contracts), which will mean that there will exist some $0<k<1$ such that, for any $P, Q \in U$ we have

$$
\|f(P)-f(Q)\|<k\|P-Q\| .
$$

We then recover the result presented by Gambaudo et al. in 63. which states the possible number of periodic orbits and their possible symbolic properties.

Theorem 6.6 (63]). Let $f$ be a piecewise-smooth map as defined above satisfying

1. $f^{n}(\Sigma) \cap \Sigma=\emptyset$ for all $n>0$,

2. $f$ contracts.

Then,

i) $f$ admits 0,1 or 2 periodic orbits

ii) any periodic orbit of $f$ has an itinerary which is maximin

iii) if $f$ has two periodic orbits, then their itineraries belong to $W_{p, q}$ and $W_{p^{\prime}, q^{\prime}}$ and $p / q$ and $p^{\prime} / q^{\prime}$ are Farey neighbours.

Before discussing it, we remark that the previous Theorem was indeed stated for a more general type of piecewise-defined maps called quasi-contractions. For completeness we provide such definition in its original form:

Definition 6.7 (Quasi-contraction). Let $\left(E_{0}, d_{0}\right)$ and $\left(E_{1}, d_{1}\right)$ be two metric spaces and $F_{0} \in E_{0}$ and $F_{1} \in E_{1}$ two points. Then, a map

$$
f: E_{0} \cup E_{1} \longrightarrow E_{0} \cup E_{1}
$$

is a quasi contraction if there exists $0 \leq k \leq 1$ such that for any $(i, j) \in\{0,1\}^{2}$, $\forall P, Q \in f^{-1}\left(E_{j}\right) \cap E_{i}, \forall R \in f^{-1}\left(E_{1-j}\right) \cap E_{i}$ one has

i) $d_{j}(f(P), f(Q)) \leq k d_{i}(P, Q)$

ii) $d_{j}\left(f(P), F_{j}\right)+d_{1-j}\left(f(R), F_{1-j}\right) \leq k d_{i}(P, R)$

Despite the arbitrary dimension of the metric spaces $E_{i}$, Theorem 6.6 was stated keeping in mind the one-dimensional case. This is why the two points $F_{i}$ were considered instead of manifolds. When considering $E_{0}=(-\infty, 0]$ and $E_{1}=[0, \infty)$, then $F_{i}$ would be chosen to coincide with the boundary: $F_{0}=$ $F_{1}=0$ and we recover the type of one-dimensional maps as in Equation (2.1). 
Theorem 6.6 was proved in 60] (Theorem $A$ ) for $f$ a quasi-contraction with constant $k$ satisfying $0 \leq k \leq 1 / 2$. The version given in 63 not only extends to the case of the quasi-contractions with $0 \leq k \leq 1$, but it also provides more information; we give here a mutilated version which is enough for our purposes. It can be easily seen that both proofs also hold when one replaces the points $F_{i}$ by closed sets whose all iterates are kept connected. This is why we required condition 1. in Theorem 6.6 instead of considering general quasi-contractions. Finally, note that if $f$ is a contraction in terms of $(6.3)$ then it is automatically a quasi-contraction when choosing $E_{0}=E_{\mathcal{L}}, E_{1}=E_{\mathcal{R}}$ and the switching manifold $\Sigma$ instead of the points $F_{i}$, and using

$$
d(f(P), \Sigma)=\min _{x \in \Sigma} d(x, f(P))
$$

(similarly for $f(Q)$ ). Hence, conditions 1. and 2. in Theorem 6.6 replace the quasi-contracting condition for the particular case of the type of piecewisesmooth maps that we are considering here.

Note that, obviously, Theorem 6.6 also holds when considering $f$ a quasicontraction for some $F_{0}=F_{1} \in \Sigma$. However, this becomes much more resctrictive than conditions 1 . and 2. .

We now analyze Theorem 6.6 keeping an analogy with the results summarized in Section 2 for the one-dimensional case.

We first note that the case in which only a periodic orbits exists is the analogous to the orientation-preserving case for one-dimensional maps. Although this is not stated in the result itself, this comes from the proof provided in 60, 63. By Proposition 6.4 such periodic orbit must have a symbolic sequence which belongs to the Farey tree of symbolic sequences (see Figure 3.5(b)].

As it also occurs for the one-dimensional case, when the map is non-orientable one finds the possibility of coexistence between two periodic orbits. As before, this comes from the proof provided in 60,63 . Let $\beta$ and $\Delta$ be the symbolic sequences of those periodic orbits. From iii) $(\beta)^{\infty} \in W_{p, q}$ and $(\Delta)^{\infty} \in W_{p^{\prime}, q^{\prime}}$ with $p / q$ and $p^{\prime} / q^{\prime}$ Farey neighbours at some Farey sequence $\mathcal{F}_{n}$ (see Definition 3.1). Assume that $q^{\prime}>q$, then they are neighbours at the Farey sequence of order $q^{\prime}$ and $p / q$ is a Farey parent of $p^{\prime} / q^{\prime}$. Then, if $p / q>p^{\prime} / q^{\prime}$, by Proposition 3.33 we know that $\Delta=\alpha \beta$, where $\alpha$ is the Farey sequence of the other Farey parent of $p^{\prime} / q^{\prime}$. Then, by going down through the Farey tree of symbolic sequences we find two sequences, $\sigma$ and $\gamma$ such that $\beta=\sigma^{n} \gamma$ and $\Delta=\sigma^{n+1} \gamma$, for some $n \geq 0$. Then, by considering the iterates of the maps $f_{\mathcal{L}}$ and $f_{\mathcal{R}}$ given by the sequences $\sigma$ and $\gamma$ (see the example bellow), the periodic orbits of the original map are collapsed to periodic orbits of the form $\mathcal{L}^{n+1} \mathcal{R}$ and $\mathcal{L}^{n} \mathcal{R}$ (or $\mathcal{L} \mathcal{R}^{n+1}$ and $\mathcal{L} \mathcal{R}^{n}$ ) for the composite map, which corresponds to the coexistence of periodic orbits given at the period incrementing structure.

Example 6.8. Assume that we are in the situation described in iii) with two periodic orbits with symbolic sequences in $W_{3,8}$ and $W_{2,5}$ coexisting. From Section 3.4 we know that, with the notation from above, these symbolic sequences 
are $\Delta=\left(\mathcal{L}^{2} \mathcal{R}\right)^{2} \mathcal{L} \mathcal{R}$ and $\beta=\mathcal{L}^{2} \mathcal{R} \mathcal{L} \mathcal{R}$. By going down trough the tree, we get $\alpha=\sigma=\mathcal{L}^{2} \mathcal{R}$ and $\gamma=\mathcal{L} \mathcal{R}$. Then, for two properly chosen sets $\tilde{E}_{\mathcal{L}}$ and $\tilde{E}_{\mathcal{R}}$ (see below for more details), the map

$$
\tilde{f}(x)= \begin{cases}\tilde{f}_{\mathcal{L}}(x):=f_{\mathcal{L}}^{2} \circ f_{\mathcal{R}}(x) & \text { if } x \in \tilde{E}_{\mathcal{L}} \\ \tilde{f}_{\mathcal{R}}(x):=f_{\mathcal{L}} \circ f_{\mathcal{R}}(x) & \text { if } x \in \tilde{E}_{\mathcal{R}}\end{cases}
$$

possesses two periodic orbits with symbolic sequences $\mathcal{L}^{2} \mathcal{R}$ and $\mathcal{L} \mathcal{R}$, which corresponds to the predicted ones by the period incrementing bifurcation structure. Note that the sets $\tilde{E}_{\mathcal{L}}$ and $\tilde{E}_{\mathcal{R}}$ need to be properly found. This can be done by noting that the iterates by $\tilde{f}_{\mathcal{L}}$ or $\tilde{f}_{\mathcal{R}}$ provide the boundaries of the domains of attraction between the points of the periodic orbits. See [26] and [25] for explicit examples.

Finally, the third case for which $f$ does not have any periodic orbit corresponds to quasi-periodic dynamics. In this case $f$ possesses an attracting Cantor set (see [63]).

Remark 6.9. Theorem 6.6 does not provide information about the bifurcation structures that may appear when adding parameters to $f$. As it will be discussed in Section 8, similar structures to the period adding may appear, although it is not guaranteed that all periodic orbits in the tree may exist for some parameter.

\subsection{Maximin approach for the one-dimensional case}

In this section we present an alternative path to prove the period adding structure for one-dimensional piecewise-smooth maps. More precisely we show that one can use Theorem 6.6 after Gambaudo et al. to identify sequences in the Farey tree of symbolic sequences with the itineraries of one-dimensional orientation preserving circle maps. By using the concept of maximin sequences instead of $p, q$-ordered sequences (see Definition 3.29), this approach provides stronger results than the one presented in Section 3.4, as they give more information about the symbolic sequences. In particular, given the rotation number, $p / q$, the maximin property permits to obtain the proper symbolic itinerary of a periodic orbit without constructing the whole Farey tree of symbolic sequences up to level $q$. Instead, one needs to find in $W_{p, q}$ which is the symbolic sequence that verifies the maximin/minimax condition (see Definition 6.1).

However, one of the counterparts to these advantages consists of the requirement of contraction, whereas, as remarked in Section 5. many of the results through circle maps (shown in Sections 3.3 and 3.4) also hold for expansive maps. Moreover, the proof of Theorem 6.6. specially for $1 / 2 \leq k \leq 1$ (see [63]), is significantly more difficult than the ones of in Section 3.4

We now specify the alternative path of this alternative proof, which is as follows. From Proposition 3.14 we get that, under conditions C.1-C.4, if the rotation number is rational, $p / q$, then a periodic orbit exists. When adding the assumption of contraction, Theorem 6.6 holds and, due to uniqueness of 
the rotation number, tells us that a unique periodic orbit must exist; moreover, its symbolic itinerary is maximin. Then, from Proposition 6.4, we get that its symbolic sequence belongs to the Farey tree of symbolic sequences. Once we get that the symbolic itineraries of orientation preserving circle maps belong to the Farey tree of symbolic sequences, one proceeds as in Section 3.4 to study the bifurcation structure when the parameters $c$ or $\lambda$ are varied in order to prove $i$ ) in Theorem 2.13 .

\section{Applications}

In Section 7.1 we present examples for which Theorem 2.13 provides a full description of the dynamics that one can find.

In the first example (Section 7.1.1), we will focus on the codimension-two bifurcation given by the simultaneous collision of two periodic orbits of onedimensional discontinuous map with the boundary. By applying Theorem 2.13 we generalize in this example the results obtained in 61, 60 by providing a precise description of the bifurcation scenario.

In the second example (Section 7.1.2 we deal with a system of control theory. We consider a first order system with a stable equilibrium point. In order to stabilize this system at another value, we perform a non-smooth control action based on relays, which is well extended technique in engineering control, and known as sliding-mode control. Theorem 2.13 holds and describes the dynamics of this system.

In Section 7.1.3 we consider a generic integrate-and-fire system submitted to a periodic forcing. Such a system consists of a hybrid model widely used in neuroscience. We show how Theorem 2.13 can be applied to describe, not only its dynamics, but relevant biological properties as the firing-rate under parameter variation. There exist in the literature other examples in neuron models from which one derives a piecewise-smooth discontinuous map such that Theorem 2.13 ii) holds; see for example [84, 125].

Section 7.2 is devoted to illustrate the results revisited in Section 6 for higherdimensional piecewise-smooth maps.

We first consider a higher-order system controlled with relays (Section 7.2.1). This represents an extension of the example shown in Section 7.1.2.

In Section 7.2.2 we consider a ZAD-controlled DC-DC boost converter. We show how symbolic dynamics given by the maximin periodic orbits describe how the saturation of a Pulse Width Modulation process is distributed along periodic cycles.

\subsection{One-dimensional examples}

\subsubsection{Codimension-two border collision bifurcations}

Our first example consists of a generic dynamical system given by a piecewisesmooth map for which, under variation of two parameters, two periodic orbits transversally collide with the boundary. In this two-dimensional parameter 
space, this is given by the crossing of two border collision bifurcation curves, and is hence a codimension-two bifurcation point. Below we will show that the map 2.1) is a normal form for such a bifurcation and hence the dynamics are given by Theorem 2.13 .

This bifurcation codimension-two bifurcation was reported as gluing bifurcation ([61, 60]) when studying figure of eight or butterfly homoclinic bifurcations for three-dimensional flows. Assuming the existence of a strong stable direction it is possible to reduce the dynamics near a homoclinic bifurcation to a twodimensional system. Then, one considers a first-return Poincaré map, which turns out to be a one-dimensional discontinuous map of the form given in Equation (2.1) (see 11, 120 for more details). Depending on the orientability of the stable and unstable manifolds, this map exhibits different configurations regarding its monotonicity near the discontinuity (see [66, 80]), leading to the cases $i$ )-iv) of Theorem 3.2 and Remark 2.14 .

Later on, in the context of simulations of piecewise-smooth dynamical systems, this co-dimension two bifurcation was rediscovered and called big bang bifurcation ([22]) as, depending on the monotonicity properties of the map, it may involve the emergence of an infinite number of bifurcation curves from the codimension-two bifurcation point at the parameter space. Such points organize the dynamics around them and have been highly reported in simulations of piecewise-smooth maps [20, 21, 23, 24, 17, 27, 28.

Also from the piecewise-smooth perspective, the bifurcation scenarios that appear around such a codimension-two bifurcation point have been recently reconsidered in 64. There the authors distinguish between the cases i) and ii) of Theorem 2.13. For the period adding structure (case $i$ )) the authors use renormalization arguments to demonstrate the existence of periodic orbits nested between regions in the parameter space. However, the proof is not complete, as it is not shown that this occurs only for regions containing periodic orbits with Farey neighbours rotation numbers, which is the main result. Regarding the period incrementing (case $i i)$ ), the authors of [64] repeat the proof provided in [18] and in Section 4 .

We now start with the example. We will show that, under non-degeneracy conditions, after collapsing the colliding periodic orbits to fixed points and performing a reparametrization, such a bifurcation can be reduced to the study of a map of the form of Equation (2.1) under variation of the parameters $\mu_{\mathcal{L}}$ and $\mu_{\mathcal{R}}$

Assume that we have a dynamical system given by

$$
x_{n+1}=f\left(x_{n}\right),
$$

with

$$
f(x)= \begin{cases}f_{\mathcal{L}}(x ; a, b) & \text { if } x<0 \\ f_{\mathcal{R}}(x ; a, b) & \text { if } x>0\end{cases}
$$

where $a, b \in \mathbb{R}$ are two parameters.

Assume that system 7.1 possesses two periodic orbits $\left(x_{0}, \ldots, x_{n-1}\right)$ and 
$\left(y_{0}, \ldots, y_{m-1}\right), x_{i}, y_{i} \in \mathbb{R}$, satisfying

$$
\begin{aligned}
f\left(x_{i}\right) & =x_{i+1}, 0 \leq i<n-1 \\
f\left(x_{n-1}\right) & =x_{0}
\end{aligned}
$$

and

$$
\begin{aligned}
f\left(y_{i}\right) & =y_{i+1}, 0 \leq i<m-1 \\
f\left(y_{m-1}\right) & =y_{0} .
\end{aligned}
$$

Note that the periodic orbits are indexed by dynamical and not spatial order, which implies that we do not necessarily have $x_{i}<x_{i+1}$ and $y_{i}<y_{i+1}$.

Assume that these periodic orbits undergo right and left border collision bifurcations at two curves, $b=\xi_{x}(a)$ and $b=\xi_{y}(a)$ in the parameter space $(a, b)$, respectively; i.e, there exist unique integers $k_{x} \in\{0, \ldots, n-1\}$ and $k_{y} \in\{0, \ldots, m-1\}$ such that, for any $a \in \mathbb{R}$,

$$
\begin{aligned}
& \lim _{b \rightarrow \xi_{x}(a)^{+}} x_{k_{x}}=0^{-} \\
& \lim _{b \rightarrow \xi_{y}(a)^{-}} y_{k_{y}}=0^{+} .
\end{aligned}
$$

Assume that these two curves intersect transversally at some point $\left(a^{*}, b^{*}\right)$. In order to write the map 77.2 into the form of Equation (2.1) for parameter values near $\left(a^{*}, b^{*}\right)$ we first need to consider a higher iterate of $f$ so that these periodic orbits become fixed points. To this end, let $\mathbf{x}=\left(\mathbf{x}_{1}, \ldots, \mathbf{x}_{n}\right) \in\{\mathcal{L}, \mathcal{R}\}^{n}$ and $\mathbf{y}=\left(\mathbf{y}_{1}, \ldots, \mathbf{y}_{m}\right) \in\{\mathcal{L}, \mathcal{R}\}^{m}$ be symbolic sequences such that

$$
\begin{aligned}
I_{f}\left(x_{k_{x}}\right) & =\mathbf{x}^{\infty} \\
I_{f}\left(y_{k_{y}}\right) & =\mathbf{y}^{\infty} .
\end{aligned}
$$

Then, consider the iterates

$$
\begin{aligned}
& \bar{f}_{\mathcal{L}}=f_{\mathbf{x}_{1}} \circ f_{\mathbf{x}_{2}} \circ \cdots \circ f_{\mathbf{x}_{n}}(x) \\
& \bar{f}_{\mathcal{R}}=f_{\mathbf{y}_{1}} \circ f_{\mathbf{y}_{2}} \circ \cdots \circ f_{\mathbf{y}_{m}}(x),
\end{aligned}
$$

and the map

$$
\bar{f}(x ; a, b)= \begin{cases}\bar{f}_{\mathcal{L}}(x ; a, b) & \text { if } x<0 \\ \bar{f}_{\mathcal{R}}(x ; a, b) & \text { if } x>0 .\end{cases}
$$

Note that one can always find proper shifts of the sequences $\mathbf{x}$ and $\mathbf{y}$ such that the domains of $\bar{f}_{\mathcal{L}}$ and $\bar{f}_{\mathcal{R}}$ become $x<0$ and $x>0$, respectively. Indeed, this occurs when the sequences $\mathbf{x}$ and $\mathbf{y}$ start with the points $x_{k_{x}}$ and $y_{k_{y}}$, respectively. See [26] and 25] for explicit examples.

These compositions collapse the periodic orbits of the maps $f_{\mathcal{L}}$ and $f_{\mathcal{R}}$ to fixed points of $\bar{f}_{\mathcal{L}}$ and $\bar{f}_{\mathcal{R}}$, respectively. There exists then a neighbourhood $\left(a^{*}, b^{*}\right) \in$ $\mathcal{U} \subset \mathbb{R}^{2}$ which is split in four subsets for which $\bar{f}$ has two fixed points, a unique positive fixed point, a unique negative fixed point and no fixed points. 
These fixed points undergo simultaneous border collision bifurcations at $(a, b)=$ $\left(a^{*}, b^{*}\right)$ in such a way that the map $\bar{f}$ becomes continuous at $x=0$ for these parameter values.

We now perform a reparametrization along the curves $\xi_{x}$ and $\xi_{y}$. The nondegeneracy conditions given by their transversal intersection will give us that, in first order, the new parameters will be equivalent to the offset parameters $\mu_{\mathcal{L}}$ and $\mu_{\mathcal{R}}$ of the general map given in Equation 2.1.

This reparametrization is given by

$$
\begin{aligned}
& \phi: \quad \mathbb{R}^{2} \quad \longrightarrow \quad \mathbb{R}^{2} \\
& \left(\mu_{\mathcal{L}}, \mu_{\mathcal{R}}\right) \longmapsto\left(a^{*}+\mu_{\mathcal{L}}+\mu_{\mathcal{R}}, \xi_{x}\left(a^{*}+\mu_{\mathcal{L}}\right)+\xi_{y}\left(a^{*}+\mu_{\mathcal{R}}\right)\right) \text {, }
\end{aligned}
$$

which maps the axis $\mu_{\mathcal{L}}=0$ and $\mu_{\mathcal{R}}=0$ to the bifurcation curves $\left(a, \xi_{x}(a)\right)$ and $\left(a, \xi_{y}(a)\right)$, respectively. Expanding in powers of $x, \mu_{\mathcal{L}}$ and $\mu_{\mathcal{R}}$ around $\left(x, \mu_{\mathcal{L}}, \mu_{\mathcal{R}}\right)=(0,0,0)$ we get

$$
\begin{aligned}
& \bar{f}_{\mathcal{L}}\left(x ; \phi\left(\mu_{\mathcal{L}}, \mu_{\mathcal{R}}\right)\right)=\bar{f}_{\mathcal{L}}(x ; \phi(0,0)) \\
& +D_{\mu_{\mathcal{L}}, \mu_{\mathcal{R}}} \bar{f}_{\mathcal{L}}\left(x ; \phi\left(\mu_{\mathcal{L}}, \mu_{\mathcal{R}}\right)\right)_{\substack{x=0 \\
\mu_{\mathcal{L}}=0 \\
\mu_{\mathcal{R}}=0}} \cdot\left(\begin{array}{c}
\mu_{\mathcal{L}} \\
\mu_{\mathcal{R}}
\end{array}\right) \\
& +O\left(\mu_{\mathcal{L}} x, \mu_{\mathcal{R}} x,, \mu_{\mathcal{L}} \mu_{\mathcal{R}}, x^{2}, \mu_{\mathcal{L}}^{2}, \mu_{\mathcal{R}}^{2}\right) \\
& D_{\mu_{\mathcal{L}}, \mu_{\mathcal{R}}}\left(\bar{f}_{\mathcal{L}}\left(x ; \phi\left(\mu_{\mathcal{L}}, \mu_{\mathcal{R}}\right)\right)\right)_{\substack{x=0 \\
\mu_{\mathcal{L}}=0 \\
\mu_{\mathcal{R}}=0}}=D_{\mu_{\mathcal{L}}, \mu_{\mathcal{R}}}\left(f\left(0 ; \phi\left(\mu_{\mathcal{L}}, \mu_{\mathcal{R}}\right)\right)\right)_{\substack{\mu_{\mathcal{L}}=0 \\
\mu_{\mathcal{R}}=0}} \\
& =\left(\frac{\partial}{\partial \mu_{\mathcal{L}}} \bar{f}_{\mathcal{L}}\left(0 ; \phi\left(\mu_{\mathcal{L}}, \mu_{\mathcal{R}}\right)\right)\right. \\
& \left.\frac{\partial}{\partial \mu_{\mathcal{R}}} \bar{f}_{\mathcal{L}}\left(0 ; \phi\left(\mu_{\mathcal{L}}, \mu_{\mathcal{R}}\right)\right)\right)_{\substack{\mu_{\mathcal{L}}=0 \\
\mu_{\mathcal{R}}=0}}
\end{aligned}
$$

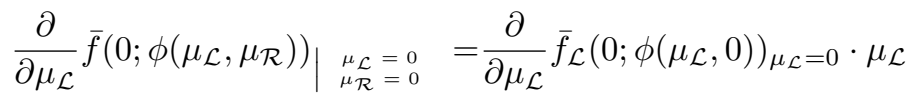

$$
\begin{aligned}
& +O\left(\mu_{\mathcal{L}}^{2}, \mu_{\mathcal{R}}^{2}, \mu_{\mathcal{L}} \mu_{\mathcal{R}}\right) \\
& \frac{\partial}{\partial \mu_{\mathcal{R}}} \bar{f}\left(0 ; \phi\left(\mu_{\mathcal{L}}, \mu_{\mathcal{R}}\right)\right)_{\substack{\mu_{\mathcal{L}}=0 \\
\mu_{\mathcal{R}}=0}}=(\frac{\partial}{\partial \mu_{\mathcal{R}}} \underbrace{\bar{f}_{\mathcal{L}}\left(0 ; \phi\left(0, \mu_{\mathcal{R}}\right)\right)}_{=0})_{\mid \mu_{\mathcal{R}}=0} \cdot \mu_{\mathcal{R}} \\
& +O\left(\mu_{\mathcal{L}}^{2}, \mu_{\mathcal{R}}^{2}, \mu_{\mathcal{L}} \mu_{\mathcal{R}}\right)
\end{aligned}
$$

Proceeding similarly for $\bar{f}_{\mathcal{R}}$ we get that there exist some constants $\delta_{\mathcal{L}}, \delta_{\mathcal{R}} \in \mathbb{R}$ such that the map $\bar{f}$ can be written, close to $\left(x, \mu_{\mathcal{L}}, \mu_{\mathcal{R}}\right)=(0,0,0)$, as

$$
\bar{f}\left(x ; \mu_{\mathcal{L}}, \mu_{\mathcal{R}}\right)=\left\{\begin{array}{c}
\delta_{\mathcal{L}} \mu_{\mathcal{L}}+\bar{f}_{\mathcal{L}}\left(x ; a^{*}, b^{*}\right)+\text { h.o.t. } \\
\delta_{\mathcal{R}} \mu_{\mathcal{R}}+\bar{f}_{\mathcal{R}}\left(x ; a^{*}, b^{*}\right)+\text { h.o.t. }
\end{array}\right.
$$


where h.o.t. $=O\left(\mu_{\mathcal{L}} x, \mu_{\mathcal{R}} x, \mu_{\mathcal{L}} \mu_{\mathcal{R}}, \mu_{\mathcal{L}}^{2}, \mu_{\mathcal{R}}^{2}\right)$. Hence, taking $\delta_{\mathcal{L}} \mu_{\mathcal{L}}$ and $\delta_{\mathcal{R}} \mu_{\mathcal{R}}$ as new parameters, the first order terms of the map $\bar{f}$ given in Equation $(7.3)$ are of the form as in map (2.1) and satisfy condition h.1.

Finally, if the two periodic orbits $\left(x_{0}, \ldots, x_{n-1}\right)$ and $\left(y_{0}, \ldots, y_{m-1}\right)$ are attracting, the maps $\bar{f}_{\mathcal{L}}$ and $\bar{f}_{\mathcal{R}}$ become contracting near $x=0$. This implies that conditions H.1-H.3 are satisfied and Theorem 2.13 can be applied to system 7.3$)$ for $\delta_{\mathcal{L}} \mu_{L} L$ and $\delta_{\mathcal{R}} \mu_{\mathcal{R}}$ small. Hence, depending on the sign of $\left(\bar{f}_{\mathcal{L}}\right)^{\prime}\left(0^{-} ; a^{*}, b^{*}\right)$ and $\left(\bar{f}_{\mathcal{R}}\right)^{\prime}\left(0^{+} ; a^{*}, b^{*}\right)$ we will get situations $\left.\left.i\right)-i v\right)$ of Theorem 2.13 and Remark 2.14 .

The periodic orbits of original map $f$ given in Equation $(7.2)$ are obained from the ones of $\bar{f}$ given by Theorem 2.13 by replacing each point $x_{i}$ by

$$
\begin{array}{ll}
x_{i} \longrightarrow\left(x_{i}, f_{\mathbf{x}_{1}}\left(x_{i}\right), f_{\mathbf{x}_{2}} \circ f_{\mathbf{x}_{1}}\left(x_{i}\right), \ldots, f_{\mathbf{x}_{n}} \circ \cdots \circ f_{\mathbf{x}_{1}}\left(x_{i}\right)\right) & \text { if } x_{i}<0 \\
x_{i} \longrightarrow\left(x_{i}, f_{\mathbf{y}_{1}}\left(x_{i}\right), f_{\mathbf{y}_{2}} \circ f_{\mathbf{y}_{1}}\left(x_{i}\right), \ldots, f_{\mathbf{y}_{m}} \circ \cdots \circ f_{\mathbf{y}_{1}}\left(x_{i}\right)\right) & \text { if } x_{i}>0 .
\end{array}
$$

The corresponding symbolic for each periodic orbit of the map $f$ sequence will be obtained by replacing

$$
\begin{gathered}
\mathcal{L} \longrightarrow \mathbf{x} \\
\mathcal{R} \longrightarrow \mathbf{y} .
\end{gathered}
$$

\subsubsection{First order sliding-mode controlled system with relays}

In our next example we consider a first order one-dimensional system ${ }^{2}$

$$
\dot{y}=f(y),
$$

such that $f$ is monotonically decreasing and has a simple zero at the origin, $f(0)=0$, which is a stable equilibrium point of system (7.4). We generalize the results presented in [50] for a linear system and in [51] for second order linear system.

We wish to stabilize system (7.4 at new equilibrium point $y^{*}$ by performing a control action $u(t, y)$ :

$$
\dot{y}=f(y)+u(t, y) .
$$

Obviously, as $f$ is monotonically decreasing, one can always choose $u$ constant (open loop control) such that $f\left(y^{*}\right)+u=0$. However, if $u$ is chosen to depend on the current value of the system, $y$, (closed loop control) then such control action will be robust to small perturbations and inaccuracies on the modeling. The type of control that we will consider is based on sliding-mode. This consists on performing some action when $y>y^{*}$ and another one whenever otherwise so that solutions are "pushed" towards $y^{*}$. In a hardware setting this is implemented by means of relays. The term sliding comes from to the generalization to higher dimensions, $y \in \mathbb{R}^{n}$. In this case, one defines a surface $\sigma(y)=0$ and the desired behaviour consists of sliding motion along this surface. To achieve this, one acts similarly depending on whether $\sigma(y)>0$ or $\sigma(y)<0$.

\footnotetext{
${ }^{2}$ In this section we abuse notation and refer with $f$ to a field rather than a map.
} 


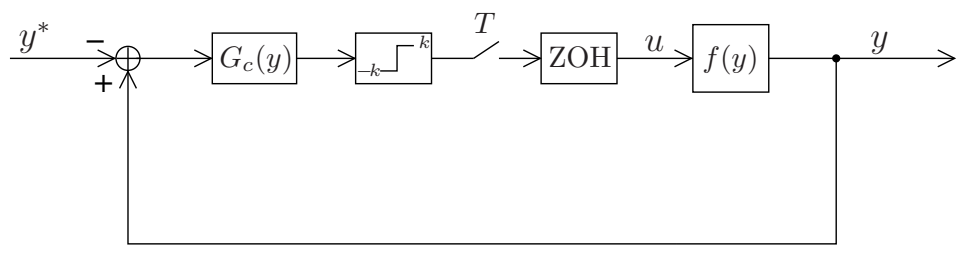

Figure 7.15: Schematic representation of the control strategy based on sliding and relays.

In addition, we will also assume that $u$ depends on $t$, as we will perform a periodic sampling in order to discretize the system and use a digital control.

All together, we will show that the dynamics of such system are given by a piecewise-smooth map of the form (2.1) satisfying $h .1-h$.3. Hence, Theorem 2.13 can be applied to accurately describe the dynamics of system 7.5 .

We now describe how the control action $u$ is defined and how we derive the piecewise-smooth map.

The control is schematically illustrated in Figure 7.15. As one can see, this is a closed loop system, whose input is the desired new equilibrium $y^{*}$. The error $y-y^{*}$ becomes the input of a controller represented by the module $G_{c}$. In this work we will assume a proportional control only. Indeed, it will not be a loss of generality if we set its gain equal to 1 (it does not perform any action), provided that the next module, the relay, will also have some tunable gain.

The output of the controller is pushed to a relay of gain $k$. This means that, depending on the sign of its input, the relay will set its output to $k$ or $-k$ depending on whether $y-y^{*}>0$ or $y-y^{*}$, respectively.

The parameter $T$ is the sampling period, which digitalizes the system. At every time $T$ the switch closes and provides a new sample to the $Z O H$ (Zero Order Holder) block. This block holds the received value until it is changed at the next sampling. The input of the module $f(y)$ becomes hence a piecewise-constant function:

$$
u(t)= \begin{cases}-k & \text { if } y(n T)-y^{*}<0 \\ k & \text { if } y(n T)-y^{*}>0\end{cases}
$$

for $t \in[n T,(n+1) T)$.

In order to obtain sliding motion we require $f\left(y^{*}\right)+k<0$ and $f\left(y^{*}\right)-k>0$; that is, we require the field 7.5 to point towards $y^{*}$. This condition can be generalized to higher dimensions in terms of the so-called equivalent control and Lie derivatives (see [129] for details). In our case, this implies that necessary $k<0$, although note that this is not a sufficient condition.

The fact that the variable $y$ is periodically sampled suggests that the dynamics of system (7.5) with $u$ given by Equation (7.6) can be better represented by a 
map

$$
y_{n+1}=P\left(y_{n}\right),
$$

where $y_{k}=y(n T)$ is the value of $y$ at the $n$th sample, and $P$ is the stroboscopic (time- $T$ return map) of system (7.5, 7.6). It becomes the piecewise-smooth map

$$
P(y)= \begin{cases}P_{\mathcal{L}}(y):=\varphi(T ; y ;-k) & \text { if } y<y^{*} \\ P_{\mathcal{R}}(y):=\varphi(T ; y ; k) & \text { if } y>y^{*}\end{cases}
$$

where $\varphi(t ; y ; k)$, satisfying $\varphi(0 ; y ; k)=y$, is the flow associated with the system $\dot{y}=f(y)+k$. Note that $P(y)$ is a smooth map as regular as the flow $\varphi$ if $y>y^{*}$ or $y<y^{*}$, and it is discontinuous at $y=y^{*}$ if $k \neq 0$.

Due to the monotonicity of $f$ the maps $P_{\mathcal{L}}$ and $P_{\mathcal{R}}$ are contracting for $y \in \mathbb{R}$. Moreover, they are both increasing (they preserve orientation), as they are given by integration of an autonomous differential equation. Finally, if $k<0$ is such that the sliding condition is satisfied, we get that $P_{\mathcal{L}}\left(y^{*}\right)>y^{*}$ and $P_{\mathcal{R}}\left(y^{*}\right)<y^{*}$, and $P$ undergoes a negative gap at $y=y^{*}$. Due to the monotonicity of $f$, this will occur for any $k<0$ such that $|k|$ is large enough. Let us now fix $k<0$ and we study the dynamics of the system under variation of the parameter $y^{*}$, the desired output. If $|k|$ is large enough such that the sliding condition is satisfied, then the attracting fixed points $y_{\mathcal{L}}$ and $y_{\mathcal{R}}$ of $P_{\mathcal{L}}$ and $P_{\mathcal{R}}$, respectively, are virtual:

$$
y_{\mathcal{R}}<y^{*}<y_{\mathcal{L}} .
$$

Therefore, when $y^{*}$ is varied, these fixed points may undergo border collision bifurcations when

$$
\begin{gathered}
y_{\mathcal{R}}\left(k_{\mathcal{R}}\right)=y^{*} \\
y_{\mathcal{L}}\left(k_{\mathcal{L}}\right)=y^{*} .
\end{gathered}
$$

After applying the change of variables $z \longmapsto y-y^{*}$, the one-parameter family of maps $\tilde{P}_{y^{*}}(z):=P\left(z+y^{*}\right)-y^{*}$ is in the class of maps given by applying the reparametrization (2.7) to the piecewise-smooth map (2.1) satisfying $i$ ) of Theorem 2.13. Hence, when varying $y^{*}$ from $y_{\mathcal{R}}$ to $y_{\mathcal{L}}$, one observes periodic orbits following the period adding structure.

As this occurs for any $k<0$, if $y^{*}$ is close enough to the equilibrium point of system (7.4), $y=0$, the origin of the parameter space $\left(y^{*}, k\right)$ represents a co-dimension two bifurcation point. This is shown in Figure 7.16 for the linear system

$$
f(y)=a y
$$

with $a<0$. The bifurcation curves shown in Figure 7.16(a) are straight lines due to the linearity of the system.

In the parameter region where one finds the period adding structure is the one for which the system performs the proper tracking. Its asymptotic dynamics consists of periodic orbits bouncing around the desired equilibrium point and whose amplitude can be made arbitrarily small by choosing $T$ small enough. For any chosen $y^{*}$, there exists a maximal value of $k$ for which the systems performs 


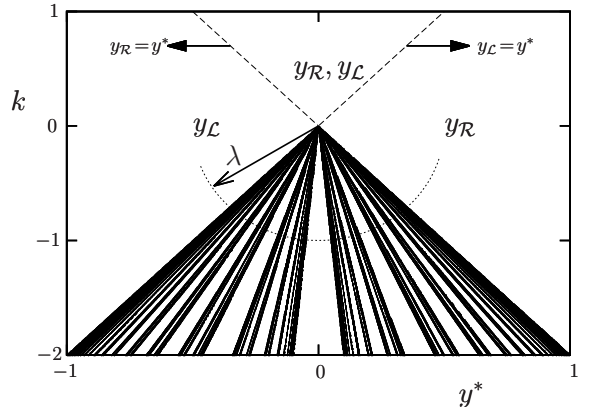

(a)

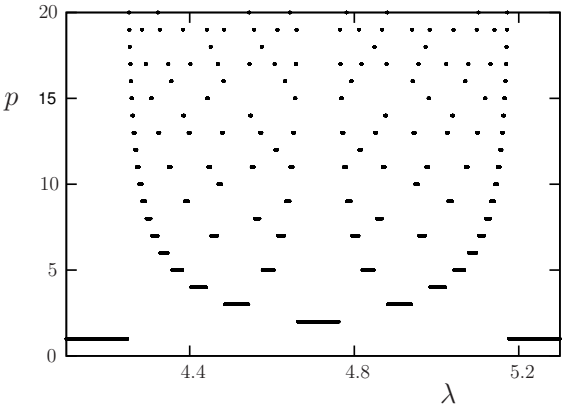

(b)

Figure 7.16: (a) Co-dimension two bifurcation point of the adding type for a the system $f(y)=-0.2 y$ and $T=0.1$. The fixed points $y_{i}$ are labeled in the regions where they are feasible, and, as dashed lines, the curves where they undergo border collision bifurcation. In (b) we show the periods $p$ of the periodic orbits found along the pointed curve in (a), which is parametrized by the angle $\lambda$.

tracking. However, although for larger values of $k$ the system oscillates between the desired valued $y^{*}$, not only the amplitude of the periodic motion grows, but also its symbolic dynamics, which can be modified by properly tunning $k$ such that a certain behaviour is achieved (or avoided). For example, one may wish to minimize the time for which the system's output exceeds $y^{*}$, and hence one may choose symbolic sequences with low rotation number.

\subsubsection{Hybrid systems in biology}

In this example we consider a generalization of an integrate-and-fire system, widely used neuron model. It consists of a hybrid system given by ${ }^{3}$

$$
\dot{x}=f(x),
$$

$f \in C^{\infty}(\mathbb{R})$, submitted to the reset condition

$$
x=\theta \longrightarrow x=0 ;
$$

that is, whenever the variable $x$ reaches a certain threshold $\theta$, it is reset to a certain value, which we assume to be $x=0$. This emulates spike of a neuron (action potential).

\footnotetext{
${ }^{3}$ As in Section 7.1 .2 in this section we abuse notation and refer with $f$ to a field rather than a map.
} 
Typically, the dynamics of system (7.7)-(7.8) are studied by means of the socalled firing map ([89), which is a Poincaré map onto the threshold $x=\theta$. However, when system (7.7) is periodically forced, this map is not optimal for obtaining general results, as one has to explicitly compute the time when spikes occur.

In this example we present the results reported in [74, where it was shown that, when a periodic forcing is considered, it is more convenient to study the system by means of the stroboscopic map (time- $T$ return map, being $T$ the period of the forcing).

Following [74, in this example we periodically force system 7.7 by means of a square wave function, which models a pulsatile stimulus of a neuron. That is, we consider the system

$$
\dot{x}=f(x)+I(t), x \in \mathbb{R}
$$

with $f(x) \in C^{\infty}(\mathbb{R})$ and $I(t)$ the $T$-periodic function

$$
I(t)= \begin{cases}A & \text { if } t \in(n T, n T+d T] \\ 0 & \text { if } t \in(n T+d T,(n+1) T],\end{cases}
$$

where $A>0$ and $0 \leq d \leq 1$ is the so-called duty cycle. We are interested in the bifurcation structure in the parameter space given by the amplitude of the pulse, $A$, and its duty cycle, $d$. We refer to [73] for the study of the bifurcation structures in this parameter space under frequency variation of the input, $1 / T$.

Let us assume that system (7.7) satisfies

A.1) it possesses an attracting equilibrium point

$$
0<\bar{x}<\theta
$$

A.2) $f(x)$ is a monotonic decreasing function in $[0, \theta]$ :

$$
f^{\prime}(x)<0, x \in[0, \theta] .
$$

Note that, conditions A.1-A.2 guarantee that spikes can only occur when the pulse is active $(I=A)$ with $A$ large enough; otherwise, when the pulse is off, trajectories are attracted by the equilibrium point $\bar{x}$.

As mentioned above, by contrast to typical approaches by means of the firing map, we use the stroboscopic map:

$$
\begin{array}{rlc}
\mathfrak{s}:[0, \theta) & \longrightarrow & {[0, \theta)} \\
x_{0} & \longmapsto \phi\left(T ; x_{0}\right),
\end{array}
$$

where $\phi\left(t ; x_{0}\right)$ is the solution of system (7.8)-(7.10) with initial condition $\phi\left(0 ; x_{0}\right)=$ $x_{0}$. Note that the flow $\phi$ is well defined, although it is discontinuous when spikes occur. The stroboscopic map $\mathfrak{s}$ is a piecewise-smooth map. To see this, we define the sets

$$
\begin{gathered}
S_{n}=\left\{x_{0} \in[0, \theta) \text { s.t. } \phi\left(t ; x_{0}\right) \text { reaches the threshold }\{x=\theta\}\right. \\
n \text { times for } 0 \leq t \leq T\}, n \geq 0 .
\end{gathered}
$$


When restricted to $S_{n}$, the map $\mathfrak{s}$ becomes a concatenation of maps consisting of integrating the system $\dot{x}=f(x)+A$ and performing resets, for $t \in[0, d T]$, and then integrating the system $\dot{x}=f(x)$ for $t \in(d T, T]$. Recall that, as mentioned above, no resets can occur for $t \in(d T, T)$. Hence, $\mathfrak{s}$ is smooth in the interior of $S_{n}$, as it is given by a certain composition of smooth maps; however, $\mathfrak{s}$ is discontinuous at the boundaries of $S_{n}$. This comes from the following fact. Let us define $\Sigma_{n} \in S_{n}$ as the initial condition that leads to a trajectory exhibiting its $n$th spike precisely when the pulse is deactivated:

$$
\phi\left(d T ; \Sigma_{n}\right)=\theta .
$$

Note that, as the flow $\phi\left(t ; x_{0}\right)$ of system 7.8$)-(7.10)$ is well defined if it exists, such initial condition is unique and can only exist for certain $n$. Notice also that, if there exists $\Sigma_{n} \in(0, \theta)$, then points above $\Sigma_{n}$ exhibit $n$ spikes, whereas points below exhibit $n-1$ spikes (see Figure 7.17).

As a consequence, the map $\mathfrak{s}$ undergoes at most one discontinuity, at $x=\Sigma_{n}$, and points to its left and right exhibit $n-1$ and $n$ spikes, respectively, for $t \in[0, d T]$. As shown in [74, for any $n$ the discontinuity $\Sigma_{n}$ satisfies

$$
\frac{d \Sigma_{n}}{d A}<0
$$

and hence it decreases montonically with $A$. By increasing this parameter, the discontinuity $\Sigma_{n}$ collides with $x=0$, disappears and a new discontinuity, $\Sigma_{n+1}$, appears at $x=\theta$. At this moment, the system changes from exhibiting $n-1$ and $n$ spikes to exhibiting $n$ and $n+1$ (see [74 for more details).

If we compute the lateral images of $\mathfrak{s}$ at these values are given by

$$
\begin{aligned}
& \mathfrak{s}\left(\Sigma_{n}^{-}\right)=\varphi(T-d T ; \theta ; 0) \\
& \mathfrak{s}\left(\Sigma_{n}^{+}\right)=\varphi(T-d T ; 0 ; 0),
\end{aligned}
$$

where $\varphi\left(t ; x_{0} ; A\right)$ is the flow associated with the system $\dot{x}=f(x)+A$, with initial condition $\varphi\left(0 ; x_{0} ; A\right)=x_{0}$. Note that $\mathfrak{s}\left(\Sigma_{n}^{-}\right)>\mathfrak{s}\left(\Sigma_{n}^{+}\right)$and hence $\mathfrak{s}$ undergoes a negative gap at $x=\Sigma_{n}$ (see Figure 7.17). Clearly, as the reset action and the integration of the flow provide orientation preserving maps, $\mathfrak{s}$ is an increasing map.

The stroboscopic map $\mathfrak{s}$ is always contracting in $\left[0, \Sigma_{n}\right)$. However, it is contractive in $\left[\Sigma_{n}, \theta\right)$ only if $n$ is large enough (74] Lemma 3.4).

One can see (74]) that, for any $n \geq 0$ and $d \in(0,1)$, there exists a range of values of $A,\left(A_{n}^{\mathcal{R}}, A_{n}^{\mathcal{L}}\right)$, for which $\mathfrak{s}$ possesses a unique fixed point, $\bar{x}_{n} \in S_{n} \subset$ $(0, \theta)$. These fixed points undergo border collision bifurcations at $A=A_{n}^{\mathcal{R}}$ and $A=A_{n}^{\mathcal{L}}$ when colliding with the boundaries $\Sigma_{n}$ and $\Sigma_{n-1}$, respectively:

$$
\begin{aligned}
& A \longrightarrow A_{n}^{\mathcal{L}}(d) \Longrightarrow \bar{x}_{n} \longrightarrow\left(\Sigma_{n}\right)^{-}, n \geq 0 \\
& A \longrightarrow A_{n}^{\mathcal{R}}(d) \Longrightarrow \bar{x}_{n} \longrightarrow\left(\Sigma_{n-1}\right)^{+}, n \geq 1,
\end{aligned}
$$

(see Figure 7.18 for $n=2$ ). 


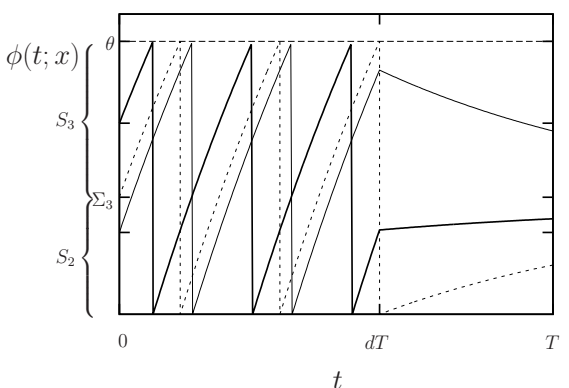

(a)

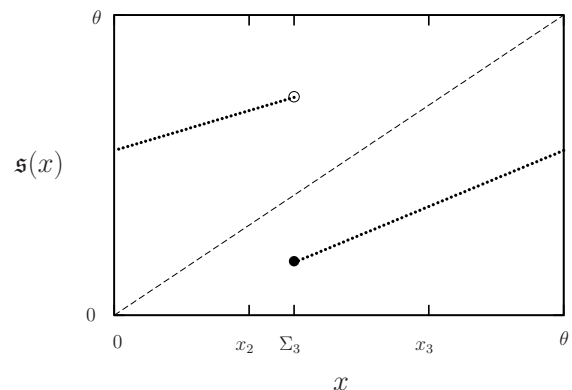

(b)

Figure 7.17: In (a) the trajectories of systems (7.8)-(7.10). Dashed line: trajectory with $\Sigma_{3}$ as initial condition. Thick line: trajectory with $x_{3}>\Sigma_{3}$ as initial condition, which spikes 3 times. Normal line: trajectory with $x_{2}<\Sigma_{3}$ as initial condition, which spikes 2 times. In (b) the stroboscopic map, with a discontinuity at $x=\Sigma_{3}$.

As given in Equation (7.13), $\Sigma_{n}$ monotonically decreases with $A$. Hence, for any $n \geq 0$, one can apply a reparametrization such that $\mathfrak{s}$ can be written as in Equation (2.1) and $A$ becomes equivalent to the parameter $\lambda$ in Equation (2.7). Therefore, if $n$ is large enough such that $\mathfrak{s}$ is contracting, conditions H.1-H.3 and i) of Theorem 2.13 are satisfied, and $\mathfrak{s}$ exhibits a period adding bifurcation structure under variation of $A \in\left[A_{n}^{\mathcal{L}}, A_{n+1}^{\mathcal{R}}\right]$ (see [74] Proposition 3.4). As this occurs for any $d \in(0,1)$, the parameter space $(d, 1 / A)$ possesses an infinite number of period adding structures. These are shown Figure 7.19 for a linear system for $f(x)$. The black regions shown in Figure 7.19(a) correspond to fixed points; for example, for parameter values at points $B, C, D$ and $E$ there exist the fixed points $\bar{x}_{0} \in S_{0}, \bar{x}_{1} \in S_{1}, \bar{x}_{2} \in S_{2}$ and $\bar{x}_{3} \in S_{3}$, respectively. As one can see from the periods of the periodic orbits shown in Figure 7.19(b), there exist period adding structures nested between the regions of existence of fixed points, as predicted by Theorem 2.13. Note however that the adding structure between $B$ and $C$ (involving the discontinuity $\Sigma_{1}$ ) is not complete. This is due to the fact that $n=1$ is not large enough to guarantee the contractiveness of $\mathfrak{s}$ at the interval $\left[\Sigma_{1}, \theta\right)$. As the map $\mathfrak{s}$ (after proper reparametrization and change of variables) satisfies conditions C.1-C.4, Proposition 3.14 holds to provide the existence of a periodic orbit. However, due to the expansiveness, periodic orbits may be unstable and not unique, and hence they are not easy to detect by direct simulation.

One of the most interesting properties that one can derive from the period 


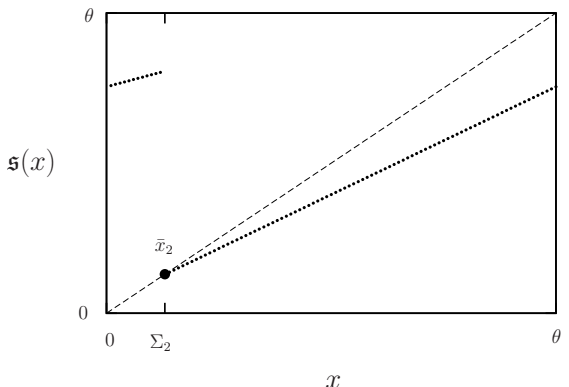

(a)

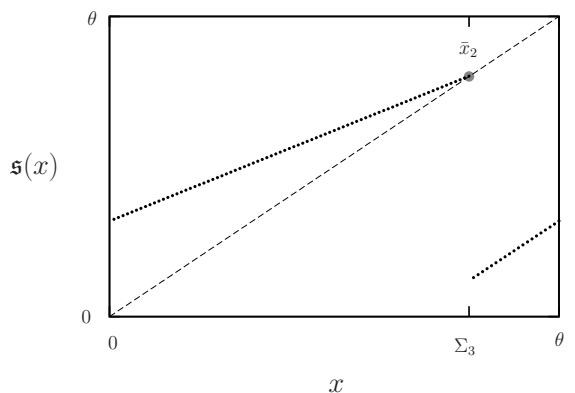

(c)

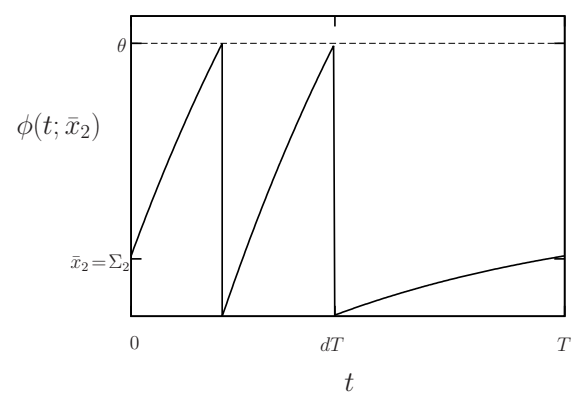

(b)

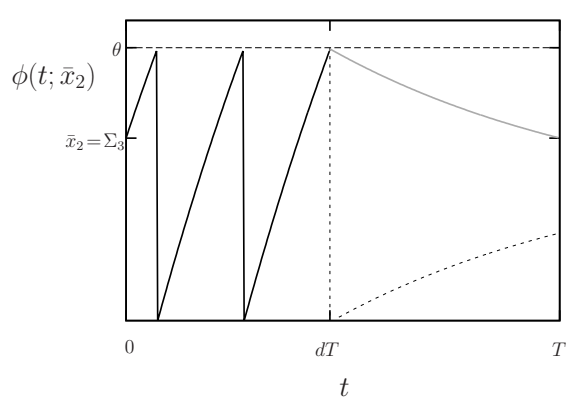

(d)

Figure 7.18: (a) Fixed point $\bar{x}_{2}$ colliding with the boundary $\Sigma_{2}$ for $A=A_{2}^{\mathcal{R}}$ (b) $T$-periodic orbit for $A=A_{2}^{\mathcal{R}}$. (c) fixed point $\bar{x}_{2}$ colliding with the boundary $\Sigma_{3}$ for $A=A_{3}^{\mathcal{L}}$. (d) $T$-periodic orbit for $A=A_{3}^{\mathcal{L}}$. Note that the periodic orbit in (d) should exhibit a reset at $t=d T$; this is why it is plot in gray color. 


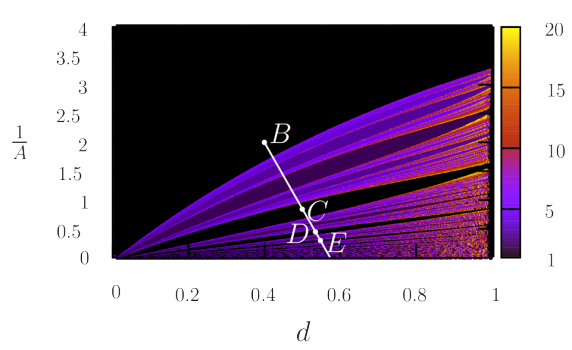

(a)

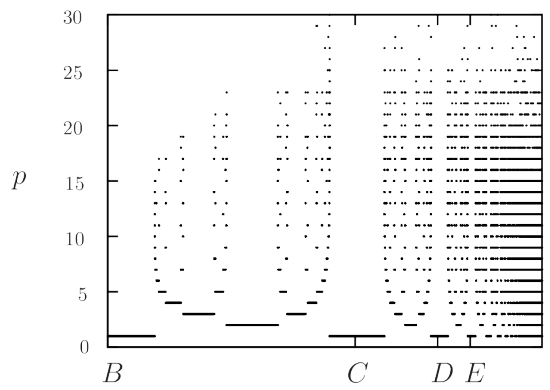

(b)

Figure 7.19: (a) Bifurcation scenario in the $(d, 1 / A)$ parameter space for the map $\mathfrak{s}$ with $f(x)=-0.5 x+0.2, \theta=1$ and $T=1.9$. The colors refer to the periods of the periodic orbits found by simulating the system. (b) period of the periodic orbits found along the the line shown in (a).

adding structures comes from the symbolic dynamics and the rotation numbers associated with these periodic orbits. Specifically, consider parameter values such that $\Sigma_{n} \in(0, \theta)$ and suppose that the stroboscopioc map has a periodic orbit. As explained above, on each iteration of the stroboscopioc map the flow can perform $n-1$ or $n$ spikes. We assign a symbolic sequence to this periodic orbit by letting its $k$ th symbol to be $\mathcal{L}$ if this number is $n-1$ and $\mathcal{R}$ if it is $n$. Hence, when divided by the period of the periodic orbit, this becomes the socalled firing number, which is the average number of spikes per iteration of the stroboscopic map. Therefore, after recalling Definition 2.11 and Corollary 3.32 . it is easy to see that the rotation number $(\rho)$ and the firing number $(\eta)$ satisfy the relation

$$
\eta=n+\rho,
$$

where $n$ is such that the periodic orbit steps around the discontinuity $\Sigma_{n+1}$, $n \geq 0$. Therefore, by Theorem 2.13, under parameter variation, the firing number follows a devil's staircase growing from 0 to infinity (see Figure 7.20). When divided by $T$ (period of the periodic forcing $I(t)$ ), the firing number becomes the so-called firing rate, which is the asymptotic average number of spikes per unit time. Hence, it also follows a devil's staircase when varying parameters $A$ and $d$ along lines as the one shown in Figure 7.19(a). However, as most of the bifurcation structures shown in Figure 7.19(a) do not qualitatively depend on $T$, when this parameter is varied the firing rate follows a devil's staircase with pieces of hyperbolas as steps. This is shown in Figure 7.20(b) and more details on this frequency analysis can be found in [73]. 


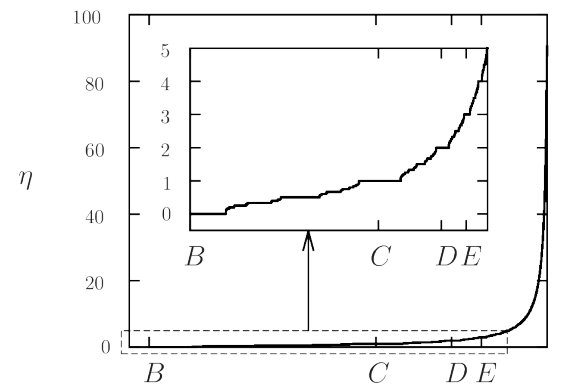

(a)

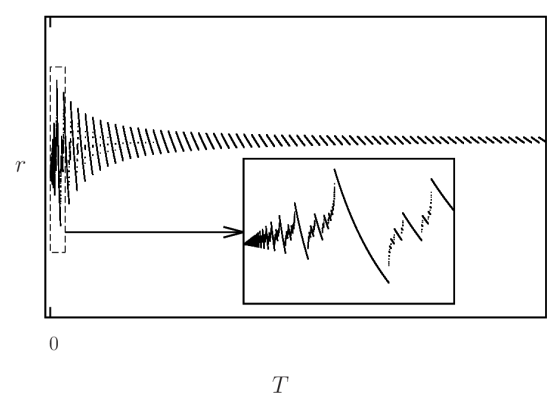

(b)

Figure 7.20: (a) Firing number along the line shown in Figure 7.19(a) for the same system. (b) Firing rate under variation of $T$.

\subsection{Examples in higher dimensions}

\subsubsection{Higher order sliding-mode controlled system with relays}

In this example we recover the results presented in [51, which extend the example shown in Section 7.1.2 to higher order linear systems.

Assume that system (7.4) is a linear system of order $n$ with an equilibrium point at the origin. In this case, due to the linearity, the block representing the open-loop system in Figure 7.15 can be replaced by its Laplace transform

$$
G_{s}(s)=\frac{b}{U(s)},
$$

where $U(s)$ is a polynomial of the form

$$
U(s)=s^{n}+a_{n-1} s^{n-1}+\cdots+a_{0},
$$

which we assume to have real negative roots only.

The system can be written in terms of a differential equation as

$$
y^{n)}+a_{n-1} y^{n-1)}+\cdots+a_{0} y=b u(t),
$$

with $y(t), a_{i}, b \in \mathbb{R}, y^{i)}=d^{i} y / d t^{i}$ and $u(t) \in \mathbb{R}$ is the input of the system. We wish to design a control such that the output of the system, $y(t) \in \mathbb{R}$, is stabilized around a new equilibrium point $y^{*}$; that is, $y(t) \simeq y^{*}$ and $y^{i)} \simeq 0$ for $1 \leq i \leq n$. To achieve this, we consider a two-step control. A first action is taken by a classic controller given by the block $G_{c}(s)$, which we assume to be of the form

$$
G_{c}(s)=1+c_{1} s+\ldots+c_{n-1} s^{n-1} .
$$


The second part will be given by a relay of gain $k$. Then, the desired motion becomes

$$
y-y^{*}+c_{1} y^{1)}+\ldots+c_{n-1} y^{n-1)}=0 .
$$

Equivalently, system 7.15 can be written as a first order $n$-dimensional system as

$$
\dot{\bar{y}}=A \bar{y}+B u,
$$

where

$$
\bar{y}=\left(y, y^{1)}, \ldots, y^{n-1)}\right) \in \mathbb{R}^{n}
$$

and $A$ and $B$ become

$$
A=\left(\begin{array}{cccccc}
0 & 1 & 0 & \ldots & & 0 \\
0 & 0 & 1 & & & 0 \\
\vdots & \vdots & & \ddots & & \\
0 & 0 & \ldots & 0 & 1 & 0 \\
-a_{0} & -a_{1} & \ldots & & -a_{n-2} & -a_{n-1}
\end{array}\right), B=\left(\begin{array}{c}
0 \\
\vdots \\
0 \\
b
\end{array}\right)
$$

and we wish to stabilize the system around the point

$$
\bar{y}=\left(\begin{array}{c}
y^{*} \\
0 \\
\vdots \\
0
\end{array}\right)
$$

After the $T$-periodic sampling, the input $u$ becomes constant at the intervals $t \in[i T,(i+1) T):$

$$
u= \begin{cases}-k & \text { if } \sigma(\bar{y})<0 \\ k & \text { if } \sigma(\bar{y})>0\end{cases}
$$

where

$$
\sigma(\bar{y})=y-y^{*}+c_{1} y^{1)}+\ldots+c_{n-1} y^{n-1)} .
$$

We wish to stabilize the system close to the switching surface

$$
\sigma(\bar{y})=0 .
$$

The fact that the polynomial $U(s)$ possesses real negative roots only ensures us that, for $k=0$, system (7.16) possesses an attracting node at the origin. In order to find proper values of $k$ such that there exists dynamics satisfying $\sigma(\bar{y}(t))=0$, we impose sliding motion on the surface given by $\sigma=0$, which occurs when the vector fields $F^{ \pm}=A \bar{y} \pm B k$, obtained by replacing $u= \pm k$, point both to the surface $\sigma$. Since $F^{ \pm}$are smooth everywhere, this can be checked through

$$
\left(L_{F^{+}} \sigma\right)\left(L_{F^{-}} \sigma\right)<0 .
$$

Let us define $u_{e q}=-\frac{(\nabla \sigma) A \bar{y}}{c_{n-1} b}$. Then, the previous inequality meets on the subset of $\sigma$ defined by

$$
-|k|<u_{e q}<|k|
$$


(see 129 for details). In turn, this result can be read as for $k$ properly selected (both in sign and in absolute value), there is sliding motion on $\sigma$.

After the discretization performed by the $T$-periodic sampling, the system is better understood by the time- $T$ return (stroboscopic) map

$$
P(y)= \begin{cases}P_{\mathcal{L}}(\bar{y}):=\rho \bar{y}+\mu_{\mathcal{L}} & \text { if } \sigma(\bar{y})<0 \\ P_{\mathcal{R}}(\bar{y}):=\rho \bar{y}+\mu_{\mathcal{R}} & \text { if } \sigma(\bar{y})>0\end{cases}
$$

where $\rho, \mu_{\mathcal{L}}$ and $\mu_{\mathcal{R}}$ are the matrices

$$
\rho=e^{A T}, \quad \mu_{\mathcal{R}}=k(\rho-I d)\left(A^{-1} B\right), \quad \mu_{\mathcal{L}}=-k(\rho-I d)\left(A^{-1} B\right) .
$$

The maps $P_{\mathcal{L}}$ and $P_{\mathcal{R}}$ are the time- $T$ return maps (stroboscopic) maps associated with the fields $F^{+}$and $F^{-}$, respectively.

Using that system $(7.16)$ is linear and that, for $k=0$, it possesses an attracting node, the matrix $\rho$ possesses real positive eigenvalues with modulus less than 1 only.

Each branch of the map (7.21), $P_{r}$ and $P_{\mathcal{L}}$, has a fixed point

$$
\bar{y}_{\mathcal{R}}=-(\rho-I d)^{-1} \mu_{\mathcal{R}}, \quad \bar{y}_{\mathcal{L}}=-(\rho-I d)^{-1} \mu_{\mathcal{L}},
$$

which may be feasible or virtual depending on whether they belong to the domain of their respective map or not. When feasible, these fixed points become attracting nodes, and they undergo border collision bifurcations when they collide with boundary $\sigma(\bar{y})=0$.

Regarding the possible dynamics, we distinguish between three different situations.

If both fixed points are feasible $\left(\sigma\left(\bar{y}_{\mathcal{R}}\right)>0\right.$ and $\left.\sigma\left(\bar{y}_{\mathcal{L}}\right)<0\right)$ they also become attracting fixed points of the map (7.21). Their domains of attraction are formed by the values of $\bar{y} \in \mathbb{R}^{n}$ such that $\sigma(\bar{y})>0$ and $\sigma(\bar{y})<0$, respectively.

If only one of both fixed points is feasible $\left(\sigma\left(\bar{y}_{\mathcal{R}}\right)<0\right.$ and $\sigma\left(\bar{y}_{\mathcal{L}}\right)<0$ or viceversa), then it becomes the unique fixed point of the map (7.21). As it is attracting, all trajectories tend towards it, and now its domain of attraction becomes $\mathbb{R}^{n}$.

Note that, in these two previous cases, the control specification is not fulfilled as $\sigma(\bar{y})=0$ is not flow invariant by the vector fields $F^{ \pm}$.

The third situation occurs when both fixed points are virtual $\left(\sigma\left(\bar{y}_{\mathcal{R}}\right)<0\right.$ and $\left.\sigma\left(\bar{y}_{\mathcal{L}}\right)>0\right)$. This occurs when the sliding condition $(7.19)$ is fulfilled, guaranteeing that the original time-continuous system possesses sliding motion on $\sigma(\bar{y})=0$. Provided that the fixed points are attracting, the map 7.21 satisfies condition 2. of Theorem 6.6. Letting

$$
\Sigma=\left\{\bar{y} \in \mathbb{R}^{n}, \mid \sigma(\bar{y})=0\right\} \cap U,
$$

condition 1 . is also satisfied by taking the set $U$ small enough but containing both virtual fixed points. As a consequence and noting that both $P_{\mathcal{L}}$ and $P_{\mathcal{R}}$ preserve orientation as they are obtained from the integration of a flow, the map 


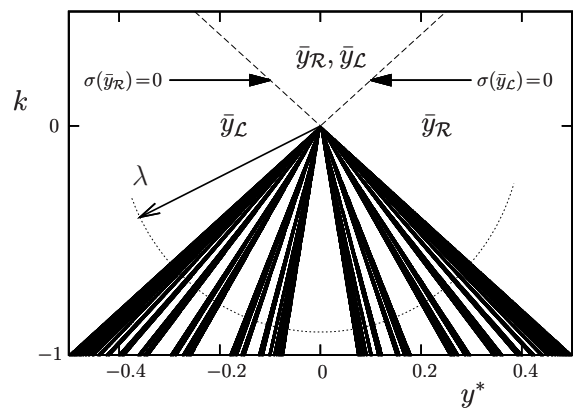

(a)

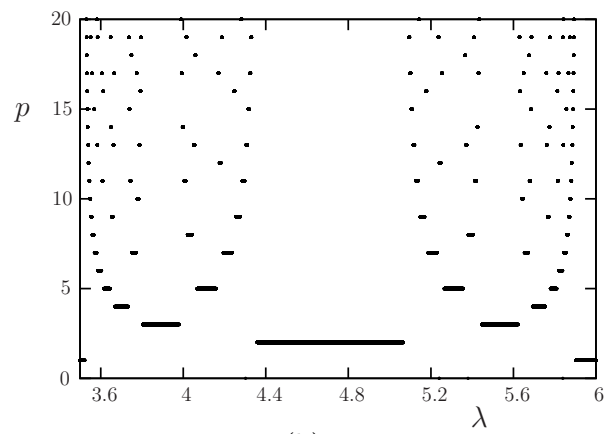

(b)

Figure 7.21: Bifurcation scenario in the $\left(y^{*}, k\right)$ parameter space for the planar case, $n=2$. The rest of parameters are fixed to $a_{0}=-2, a_{1}=-5, b=1$, $c_{1}=1.5$ and $T=0.1$. In (a) we show the border collision bifurcation curves separating existence regions of periodic orbits. In (b) the periods of the periodic orbits found along the pointed curve in (a) parametrized by $\lambda$.

$P$ admits 0 or 1 periodic orbit. When it exists, such periodic orbit must have a symbolic itinerary contained in the Farey tree of symbolic sequences shown in Figure 3.5(b).

As noted in Remark 6.9, the results given in Section 6 do not provide information about bifurcation structures. In Figure 7.21 we show the bifurcation structure obtained for the planar case when the parameters $k$ and $y^{*}$ are varied. As one can see in Figure 7.21(b) the obtained structure resembles the period adding described in Section 2.2 for the one-dimensional case, although it is not known whether for the planar case the gluing of orbits is also fractal. A similar physical interpretation of the bifurcation structure as in the first-order case (Section 7.1.2) holds also also in this case.

\subsubsection{ZAD-controlled DC-DC boost converter}

In this applied example we recover the results shown in [7] and state them in terms of the quasi-contractions of Section 6. We consider a DC-DC boost converter, which is aimed to convert a given constant DC voltage, $v_{i}$, into a desired lower one, $v_{o}$, also DC. Its circuit is schematically shown in Figure 7.22 . To achieve this conversion in a robust way (to guarantee a certain stability of $v_{o}$ under possible fluctuations of $v_{i}$ ), the transistor shown in Figure 7.22 needs to be properly controlled. There exist different approaches to design such a control; in this example we consider the so-called Zero Average Dynamics (ZAD) strategy, which is well extended in the community $([52,8,9,10,53,16,7])$. 


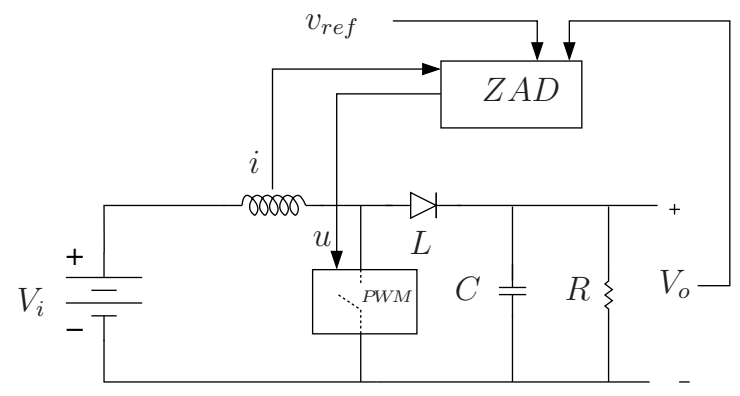

Figure 7.22: Schematic representation of a $Z A D$-controlled boost converter.

Letting $v$ be the voltage at the capacitor, $i$ the current through the solenoid, calling

$$
x=\frac{v}{v_{i}} \quad y=\sqrt{\frac{L}{C}} \frac{i}{v_{i}}
$$

and rescaling time by a factor of $\sqrt{L C}$, the non-dimensional equations that model the system become

$$
\begin{aligned}
& \dot{x}=-\gamma x+y(1-u) \\
& \dot{y}=-x(1-u)+1,
\end{aligned}
$$

with $\gamma=\sqrt{\frac{L}{R^{2} C}}$. The function $u$ takes the values 0 or 1 depending on whether the transistor is opened or closed, respectively. It is given by the output of a Pulse Width Modulation (PWM) process, and can be written as

$$
u(t)= \begin{cases}1 & \text { if } k T \leq t<k T+1 / 2 T d_{k} \\ 0 & \text { if } k T+1 / 2 T d_{k} \leq t<(k+1) T-1 / 2 T d_{k} \\ 1 & \text { if }(k+1) T-1 / 2 T d_{k} \leq t<(k+1) T\end{cases}
$$

where $d_{k} \in[0,1]$ is the so-called duty cycle. It is computed at each sampling moment, $t_{k}=k T$, and is kept constant until the next sampling period, $t_{k+1}$. Note that, if $d_{k}=1$ or $d_{k}=0$, then $u$ becomes constant in the time interval $[k T,(k+1) T)$, equal to 1 or 0 , respectively.

The ZAD strategy consists of computing the value of the duty cycle at each $T$-time interval, $d_{k}$, by imposing

$$
\int_{k T}^{(k+1) T} s(t) d t=0, \forall k \in \mathbb{Z}
$$

where $s(t)$ is the error surface

$$
s(x(t), y(t))=k_{1}\left(x(t)-x_{r e f}\right)+k_{2}\left(y(t)-y_{r e f}\right),
$$


with

$$
x_{r e f}=\frac{v_{r e f}}{v_{i}}, \quad y_{r e f}=\left(x_{r e f}\right)^{2} \gamma .
$$

and $k_{1}$ and $k_{2}$ two control constants.

Equation 7.24 becomes transcendental in $d_{k}$. However, if the solution of system 7.23 is approximated by piecewise-linear functions, the duty cycle can be approximated by the closed expression

$$
d_{k}=\frac{2 s_{0}+T \dot{s}_{2}}{\dot{s}_{2}-\dot{s}_{1}}
$$

where

$$
\begin{aligned}
& \dot{s}_{1}=-\gamma k_{1} x(k T)+k_{2} \\
& \dot{s}_{2}=k_{1}(-\gamma x(k T)+y(k T))+k_{2}(1-x(k T)) \\
& s_{0}=k_{1}\left(x(k T)-x_{r e f}\right)+k_{2}\left(y(k T)-y_{r e f}\right),
\end{aligned}
$$

(see 7 for more details). Provided that the duty cycle must be in the interval $[0,1]$, it is set to 1 or 0 depending on whether the result of expression 7.25 is greater than 1 or less than 0 , respectively. That is, one imposes the saturation condition

$$
d_{k}= \begin{cases}1 & \text { if } \frac{2 s_{0}+T \dot{s}_{2}}{\dot{s}_{2}-\dot{s}_{2}} \geq 1 \\ 0 & \text { if } \frac{2 s_{0}+T \dot{s}_{2}}{\dot{s}_{2}-\dot{s}_{2}} \leq 0 .\end{cases}
$$

Let us now consider the time- $T$ return (stroboscopic) map of system 7.23). It becomes the composition of three stroboscopic maps consisting of flowing system 77.23$)$ for the time intervals $\left[k T, k T+1 / 2 T d_{k}\right),\left[k T+1 / 2 T d_{k},(k+1) T-\right.$ $\left.1 / 2 T d_{k}\right)$ and $\left[(k+1) T-1 / 2 T d_{k},(k+1) T\right)$ setting $u=1, u=0$ and $u=1$, respectively (see 7 for more details). As these three maps are smooth maps, their composition is also a smooth map, as long as $d_{k}$ given by Equation 7.25 ) lies in the interval $(0,1)$. However, due to the saturation condition 7.26$)$, the stroboscopic map becomes indeed a piecewise-defined map, with two switching manifolds,

$$
\begin{aligned}
& \Sigma_{1}=\left\{(x, y), \mid d_{k}(x, y)=1\right\} \\
& \Sigma_{0}=\left\{(x, y), \mid d_{k}(x, y)=0\right\} .
\end{aligned}
$$

Hence, the stroboscopic map is indeed defined in three different partitions.

In Figures 7.23 and 7.24 we show the numerical results obtained by direct simulation of the stroboscopic map, by fixing the initial conditions to $\left(x_{0}, y_{0}\right)=$ $\left(2.5,(2.5)^{2} \gamma\right)$ and varying the parameters $k_{1}$ and $k_{2}$. In Figure 7.23 we show the bifurcation curves in the parameter space $\left(k_{1}, k_{2}\right)$. Due to the linearity of the system, these becomes straight lines. In Figure 7.24 we show the results obtained along the curve labeled in Figure 7.23 and parametrized by the angle $\lambda$. As shown in Figure 7.24(a), for $\lambda \in(0,0.27), \lambda \in(0.95,1.2)$ and $\lambda \in(2.5,3)$ (approximately), the stroboscopic map possesses a fixed point. For the first and 


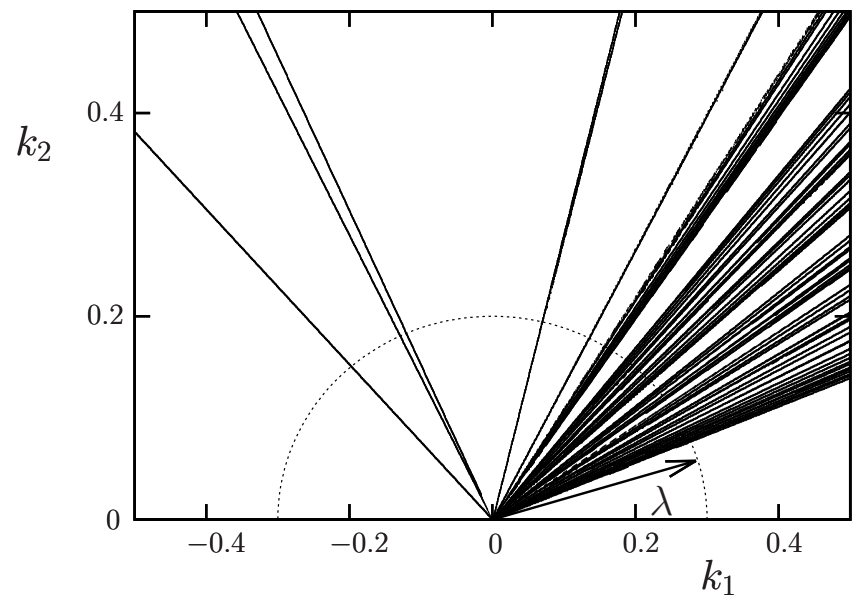

Figure 7.23: Bifurcation curves in the $k_{1} \times k_{2}$ parameter space.

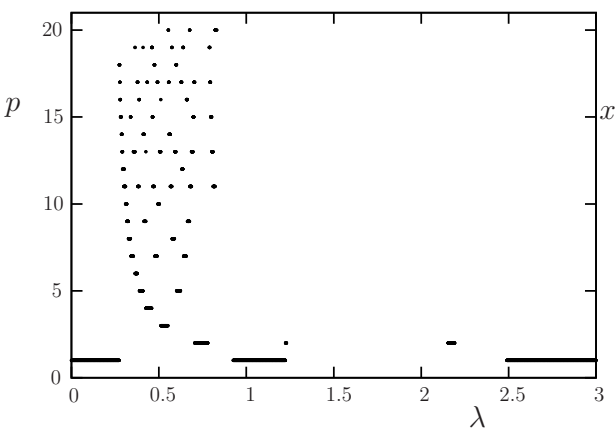

(a)

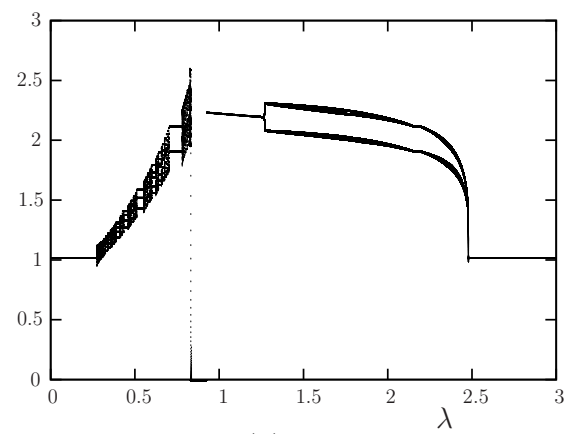

(b)

Figure 7.24: Bifurcation scenario found around the curved labeled in Figure 7.23 and parametrized by $\lambda$, for parameter values $\gamma=0.35, x_{\text {ref }}=2.5, T=0.18$. In (a) we show the periods of the periodic orbits found when varying $\lambda$. In (b) we show the bifurcation diagram corresponding to $x$. 
third intervals, the duty cycle associated with the fixed points is saturated to 0 , whereas for the second one the duty cycle lies in the interval $(0,1)$. As one can see in Figure 7.24(a), there exist a period adding-like structure between the first two intervals. The periodic orbits that one finds there possess points in the partitions where the duty cycles are saturated to 1 and to 0 . As a consequence, associated to each periodic orbit, there exists a sequence of 0 's and 1's, which are distributed such that such sequences are maximin (see Definition 6.1), or, equivalently, they belong to the Farey tree of symbolic sequences shown in Figure 3.5(b) For example, for the two found 5-periodic orbits one gets the sequences of duty cycles $(0,0,0,0,1)$ and $(0,0,1,0,1)$, which are maximin. Note that, when $d_{k}$ is saturated to 1 , the transistor remains closed for a whole sampling period and more energy is drained from the source. Therefore, if periodic orbits with high rotation number are not desired, as they possess.

Although a more accurate study of the properties of the stroboscopic map is needed, provided that the map contracts, there is evidence that the stroboscopic map is a quasi-contraction, at least for those values of $\lambda$ corresponding to the period adding-like structure.

Finally, note that for values of $\lambda$ in $(1.2,2.5)$, trajectories with the used initial condition converge towards a chaotic attractor or are aperiodic.

\section{Conclusions and future directions}

In the recent years, piecewise-smooth maps, both in $\mathbb{R}$ and in $\mathbb{R}^{n}$, have been widely investigated and very interesting bifurcation phenomena (border collisions, big bangs, period adding, period incrementing,...) have been reported by many authors. This included both works by a more applied community, like in power electronics $(7,8,9,10,19,30,43,76,87,115)$, control theory $(46,50,51,102,135,133,134,136)$, biology $([57,106)$, neuroscience $(73$, 74, 84, 88, 89, 103, 122, 123, 124, 125]) or economy ([126]) but also researchers from a more theoretical and/or computational perspective in non-smooth systems $([12,13,14,15,17,18,20,21,22,23,24,25,26,27,28,29,32,48,64,65$, 79, 93, 101, 108, 113, 126, 127). It is well known that some of these bifurcations resemble phenomena observed and highly studied in other contexts, specially in theory for circle maps in the 80's and early 90's (11, 2, 3, 4, 5, 6, 11, 32, 34, 40, 59, 60, 61, 63, 66, 86, 100, 104, 114, 121, 131, 132) and homoclinic bifurcations $(62,80,81,82,112,120,128$.

In this survey article we have considered a general setting for piecewisesmooth contracting maps with a discontinuity at the origin and exhibiting a co-dimension two border collision bifurcation. It is well known that, depending on the sign of the slopes of the map near the discontinuity, one finds mainly two different bifurcation scenarios: the period adding (for the increasing-increasing case) and the period incrementing (when the slopes have different sign). In this survey article we have revisited the latter and shown a path through the literature providing a rigorous proof for the former. Moreover, we have discussed up to which extend these results can be applied to piecewise-smooth expanding 
maps and to higher dimensions. Finally, we have shown how they can be used to provide a rigorous basis in applied examples.

\subsection{Period adding for contracting one-dimensional piecewise- smooth maps}

The main contribution of this review article consists of revisiting the literature to provide sufficient conditions for the occurrence of this bifurcation scenario (described in Section 2.2). It occurs when the map preserves orientation near the discontinuity at the origin (is of the increasing-increasing type), and the proof in summarized in Section 3.2. The key step is to link such type of maps with maps onto the circle. Although the resulting circle map is also discontinuous, the fact that the map is contracting guarantees that its lift is a strictly increasing map, which is a sufficient condition for the main properties of the rotation number of a map to hold: existence, uniqueness and continuity and monotonicity with respect to parameters. By using the concept of "well ordered" cycles, periodic orbits are linked with the symbolic sequences given in the Farey tree of symbolic sequences. When parameters are varied, theory for expanding circle maps is applied to its inverse to show that the rotation number follows a devil's staircase and, when the rotation number is irrational (which occurs in a Cantor set of zero measure), the omega limit of the map becomes rather the whole circle or a Cantor set. The latter occurs when the obtained circle map is discontinuous, the former may occur otherwise.

\subsection{Period incrementing for one-dimensional piecewise- smooth maps}

The period incrementing scenario occurs when the piecewise-smooth map has different monotonicity at both sides of the discontinuity at the origin. We recover results in the literature that provide sufficient conditions for the contracting case. The bifurcation scenario leads to the existence of stable periodic orbits of the type $\mathcal{L}^{n} \mathcal{R}$ or $\mathcal{L R}^{n}$, depending on whether the map is increasingdecreasing or decreasing-increasing, respectively. Moreover, such a periodic orbit may coexist with another periodic orbit of the type $\mathcal{L}^{n+1} \mathcal{R}\left(\right.$ or $\mathcal{L} \mathcal{R}^{n+1}$ ). In this case, both existing periodic orbits are stable and their domains of attraction are separated by the origin (the discontinuity).

\subsection{Period adding and incrementing bifurcations in piecewise- smooth expanding maps}

Most of the results regarding the orientable case (increasing-increasing) shown in this review rely on the fact that the lift of the corresponding circle map is a monotonically increasing map, and indeed do not require the map to be contracting. A necessary condition becomes then the reversibility of the map, which is guaranteed when it contracts near the discontinuity. However, such 
reversibility condition also holds when the map is not too expanding. In particular, if this holds, the map possesses the same type of periodic orbits as in the contracting case, although these may be not unique nor stable. As opposite to the contracting case, the set of parameters for which one finds dynamics associated with irrational rotation numbers becomes of non-zero measure.

When the map becomes enough expanding so that the reversibility condition no longer holds, one loses the uniqueness of the rotation number and needs to deal with rotation intervals instead. Although there is large theory and literature on this $([1,2,4,4,68,70,132])$, more work is needed in order to provide general sufficient conditions leading to precise and concrete description of the possible bifurcation scenarios.

The results for the non-orientable case (period incrementing bifurcation) are based on topological arguments for maps on the interval. The type of periodic orbits perform a number of steps (re-injection number) on the domain of the increasing branch and only one in the decreasing one. The smaller the gap at the origin of the increasing branch is compared to the gap of the decreasing one, the larger the re-injection number is. Therefore, when the decreasing branch is expanding, some of the techniques can also be used when this re-injection number is large enough to compensate the expansiveness of the decreasing branch. This results on the existence of the same type of bifurcations for periodic orbits of large enough length (re-injection number). Although the same type of period orbits with shorter period may also exist even when the decreasing branch is expanding, they may be unstable and coexist with more than one periodic orbit of the type $\mathcal{L}^{n} \mathcal{R}$.

In the case where both branches of the map are expanding, the map may posses positive topological entropy and may hence be chaotic, even when the lift is continuous (105, 92, 91]).

\subsection{Maps in higher dimensions}

The jump from dimension one to dimension two represents a really challenging problem when it comes to provide general conditions for the occurrence of concrete type of bifurcations. This is magnified when they are piecewise-smooth discontinuous maps. However, as we have shown in this review article (see Section 6) some results already existent in the literature might significantly help in order to achieve such a goal. Of special interest is the concept of quasicontractions stated in the 80's by Gambaudo et al. [59, 60, 63. Although such results provide very precise description of the type of symbolic sequences associated with periodic orbits for such maps, those results heavily rely on contraction conditions. Numerical evidences (see references in Section 6.1) show that one may find similar symbolic properties under more relaxed conditions, as it occurs for the one-dimensional case.

Unfortunately, the rotation theory shown in Sections 3.3 and 3.4 cannot be exported straightforwardly for maps in higher dimensions. However, similarly as for the one-dimensional case, one may convert such maps into discontinuous maps onto higher-dimensional cylinders by identifying the images of the switch- 
ing manifolds from both sides in order to introduce a rotating behaviour. Under more restrictive conditions as for the one-dimensional case, proceeding similarly as in [36] for a particular example, this would allow one to define the lift of a map, its rotation number and obtain some theoretical results based on rotation theory.

\section{References}

[1] Ll. Alsedà and A. Falcó. On the topological dynamics and phase-locking renormalization of lorenz-like maps. Ann. Inst. Fourier, 53:859-883, 2003.

[2] Ll. Alsedà and J. Llibre. Kneading theory of lorenz maps. Dynamical systems and ergodic theory, 23:83-89, 1989.

[3] Ll. Alsedà, J. Llibre, and M. Misiurevicz. Combinatorial Dynamics and Entropy in Dimension One. World Scientific, 2000.

[4] Ll. Alsedà, J. Llibre, M. Misiurewicz, and C. Tresser. Periods and entropy for lorenz-like maps. Ann. Inst. Fourier (Grenoble), 39:929-952, 1989.

[5] Ll. Alsedà and F. Mañosas. Kneading theory and rotation intervals for a class of circle maps of degree one. Nonlinearity, 3:413-452, 1990.

[6] Ll. Alsedà and F. Mañosas. Kneading theory for a family of circle maps with one discontinuity. Acta Math. Univ. Comenianae, 65:11-22, 1996.

[7] A. Amador, S. Casanova, H.A. Granada, G. Olivar, and J. Hurtado. Codimension-two big-bang bifurcation in a zad-controlled boost dc-dc converter. IJBC, 24, 2014.

[8] F. Angulo, E. Fossas, and G. Olivar. Transition from periodicity to chaos in a pwm-controlled buck converter with zad strategy. Int. J. Bifurcation Chaos, 15(10):3245-3264, 2005.

[9] F. Angulo, G. Olivar, and M. di Bernardo. Two-Parameter DiscontinuityInduced Bifurcation Curves in a ZAD-Strategy-Controlled DC-DC Buck Converter. IEEE Trans. Circ. \& Sys. I, 55:2392-2401, 2008.

[10] F. Angulo, G. Olivar, and A. Taborda. Continuation of periodic orbits in a ZAD-strategy controlled buck converter. Chaos, Solitons and Fractals, 38:348-363, 2008.

[11] A. Arneodo, P. Coullet, and C. Tresser. A possible new mechanism for the onset of turbulence. Physics Letters, 81(4), 1981.

[12] V. Avrutin, B. Eckstein, and M. Schanz. The bandcount increment scenario. I: basic structures. Proc. R. Soc. A, 464(2095):1867-1883, 2008.

[13] V. Avrutin, B. Eckstein, and M. Schanz. The bandcount increment scenario. II: interior structures. Proc. R. Soc. A, 464(2097):2247-2263, 2008. 
[14] V. Avrutin, B. Eckstein, and M. Schanz. The bandcount increment scenario. III: deformed structures. Proc. R. Soc. A, 465(2101):41-57, 2009.

[15] V. Avrutin, B. Eckstein, M. Schanz, and B. Schenke. Bandcount incrementing scenario revisited and floating regions within robust chaos. Mathematics and Computers in Simulation (Special Issue "Discontinuous Dynamical Systems: Theory and Numerical Methods"), 2012.

[16] V. Avrutin, E. Fossas, A. Granados, and M. Schanz. Virtual orbits and two-parameter bifurcation analysis in ZAD-controled buck-converter. Nonlinear Dynamics, 63:19-33, 2011.

[17] V. Avrutin, B. Futter, and M. Schanz. The discontinuous flat top tent map and the nested period incrementing bifurcation structure. Chaos, Solitons $\&$ Fractals, 45:465-482, 2012.

[18] V. Avrutin, A. Granados, and M. Schanz. Sufficient conditions for a period increment big bang bifurcation in one-dimensional maps. Nonlinearity, 24(9):2575-2598, 2011.

[19] V. Avrutin, E. Mosekilde, Z.T. Zhusubaliyev, and L. Gardini. Onset of chaos in a single-phase power electronic inverter. Chaos, 25(4):043114, 2015 .

[20] V. Avrutin and M. Schanz. Border-collision period-doubling scenario. Phys. Rev. E, 70:026222/1-11, 2004.

[21] V. Avrutin and M. Schanz. Period doubling scenario without flip bifurcations in a one-dimensional map. Int. J. Bifurcat. Chaos, 15:1267-1284, 2005.

[22] V. Avrutin and M. Schanz. On multi-parametric bifurcations in a scalar piecewise-linear map. Nonlinearity, 19:531-552, 2006.

[23] V. Avrutin, M. Schanz, and S. Banerjee. Multi-parametric bifurcations in a piecewise-linear discontinuous map. Nonlinearity, 19:1875-1906, 2006.

[24] V. Avrutin, M. Schanz, and S. Banerjee. Codimension-3 bifurcations: Explanation of the complex 1-, 2- and 3D bifurcation structures in nonsmooth maps. Phys. Rev. E, 75:066205/1-7, 2007.

[25] V. Avrutin, M. Schanz, and L. Gardini. Calculation of bifurcation curves by map replacement. Int. J. Bifurcat. Chaos, 20(10):3105-3135, 2010.

[26] V. Avrutin, M. Schanz, and L. Gardini. Self-similarity of the bandcount adding structures: calculation by map replacement. Regular and Chaotic Dynamics, 15(6):685-703, 2010.

[27] V. Avrutin, M. Schanz, and B. Schenke. On a bifurcation structure mimicking period adding. Proc. R. Soc. A, 467(2129):1503-1518, 2011. 
[28] V. Avrutin and I. Sushko. A gallery of bifurcation scenarios in piecewise smooth 1d maps. In G.-I. Bischi, C. Chiarella, and I. Sushko, editors, Global Analysis of Dynamic Models in Economics, Finance and the Social Sciences, pages 269-395. Springer, 2013.

[29] S. Banerjee and C. Grebogi. Border collision bifurcation in twodimensional piecewise smooth maps. Phys. Rev. E, 59:4052-4061, 1999.

[30] S. Banerjee, M. S. Karthik, G. Yuan, and J.A. Yorke. Bifurcations in one-dimensional piecewise smooth maps - theory and applications in switching circuits. IEEE Trans. Circ. ES Sys. I, 47:389-394, 2000.

[31] S. Banerjee and G. C. Verghese. Nonlinear Phenomena in Power Electronics - Attractors, Bifurcations, Chaos, and Nonlinear Control. IEEE Press, 2001.

[32] C. Bernhardt. Rotation intervals of a class of endomorphisms of the circle. Proc. London Math. Soc., s3-45:258-280, 1982.

[33] F. Bizzarri, M. Storace, and L. Gardini. Bifurcation analysis of a circuitrelated generalization of the shipmap. Int. J. Bifurcation Chaos, 16:2435$52,2006$.

[34] C. Boyd. On the structure of family of cherry fields on the torus. Ergod. Th. and Dynam. Sys., 5:27-46, 1985.

[35] R. Brette. Dynamics of one-dimensional spiking neuron model. Journ. Math. Biol., 48:38-56, 2004.

[36] H. Broer, C. Simó, and J.C. Tatjer. Towards global models near homoclinic tangencies of dissipative diffeomorphisms. Nonlinearity, 11:667-770, 1998.

[37] B. Brogliato. Nonsmooth Mechanics. Springer London, 1999.

[38] V. Carmona, S. Fernández-García, F. Fernández-Sánchez, E. GarcíaMedina, and A. Teruel. Noose bifurcation and crossing tangency in reversible piecewise linear systems. Nonlinearity, 27(3):585, 2014.

[39] V. Carmona, S. Ferndández-García, F. Fernández-Sánchez, E. GarcíaMedina, and A.E. Teruel. Reversible periodic orbits in a class of 3D continuous piecewise linear systems of differential equations. Nonlinear Analysis, 75:5866-5883, 2012.

[40] P.C. Coullet, J.-M. Gambaudo, and C. Tresser. Une nouvelle bifurcation de codimension 2: le colage de cycles. C. R. Acad. Sc. Paris, série I, 299:253-256, 1984.

[41] M. Desroches, J. Guckenhiemer, B. Krauskopf, C. Kuehn, H. Osinga, and M. Wechselberger. Mixed-mode oscillations with multiple time scales. Siam Review, 54(2):211-288, 2012. 
[42] R. L. Devaney. A piecewise linear model for the zones of instability of an area preserving map. Physica D, 10:387-393, 1984.

[43] M. di Bernardo, C. J. Budd, and A. R. Champneys. Grazing, skipping and sliding: analysis of the nonsmooth dynamics of the DC/DC buck converter. Nonlinearity, 11(4):858-890, 1998.

[44] M. di Bernardo, C. J. Budd, A. R. Champneys, and P. Kowalczyk. Piecewise-smooth Dynamical Systems: Theory and Applications, volume 163 of Applied Mathematical Sciences. Springer, 2008.

[45] M. di Bernardo, F. Garofalo, L. Glielmo, and F. Vasca. Switchings, Bifurcations and Chaos in DC/DC Converters. Fundamental Theory and Applications, 45:133-141, 1998.

[46] M. di Bernardo, F. Garofalo, L. Iannelli, and F. Vasca. Bifurcations in Piecewise-Smooth Feedback Systems. Int. J. of Control, 75:1243-1259, 2002.

[47] M. di Bernardo, P. Kowalczyk, and A. Nordmark. Sliding bifurcations: a novel mechanism for the sudden onset of chaos in dry-friction oscillators. Preprint 2003.16 of Bristol Centre for Applied Nonlinear Mathematics, 2003.

[48] P. Dutta, B. Routroy, S. Banerjee, and S. Alam. On the existence of low-period orbits in $n$-dimensional piecewise linear discontinuous maps. Nonlinear Dynamics, 2007.

[49] A.F. Filippov. Differential equations with discontinuous righthand sides, volume 18. Kluwer Academic Publishers Group, 1988.

[50] E. Fossas and A. Granados. Big bang bifurcations in a first order systems with a relay. In P. Olejnik J. Mrozowski. J. Awrejcewicz, M. Kaźmierczak, editor, Proc. of Dynamical Systems Theory and Applications, pages 147$152,2011$.

[51] E. Fossas and A. Granados. Occurrence of big bang bifurcations in discretized sliding-mode control systems. Diff. Eqs. Dyn. Syst. DOI 10.1007/s12591-012-0121-y, 21:35-43, 2013.

[52] E. Fossas, R. Griñó, and D. Biel. Quasi-sliding control based on pulse width modulation, zero average dynamics and the $l_{2}$ norm. In Proc. IEEE VSS, 2000.

[53] E. Fossas, S. J. Hogan, and T. M. Seara. Two-parameter bifurcation curves in power electronic converters. Int. J. Bifurcation Chaos, 19:341$357,2009$. 
[54] D. Fournier-Prunaret, P. Chargé, and L. Gardini. Border collision bifurcations and chaotic sets in a two-dimensional piecewise linear map. Communications in Nonlinear Science and Numerical Simulation, 16(2):916 927, 2010 .

[55] A.M. Fox and J.D. Meiss. Greene's residue criterion for the breakup of invariant tori of volume-preserving maps. Physica D, 243:45-63, 2013.

[56] E. Freire, E. Ponce, and J. Ros. The focus-center-limit cycle bifurcation in symmetric 3D piecewise linear systems. SIAM J. Appl. Math, 65(6):19331951, 2005.

[57] J.G. Freire and J.A.C. Gallas. Stern-brocot trees in the periodicity of mixed-mode oscillations. Phys. Chem. Chem. Phys., 13:12191-12198, 2011.

[58] Z. Galias and X. Yu. Study of periodic solutions of discretized twodimensional sliding mode control systems. IEEE Transactions on circuits and systems-II: express briefs, 58(6):381-385, 2011.

[59] J.-M. Gambaudo. Ordre, désordre, et frontiére des systèmes Morse-Smale. PhD thesis, Université de Nice, 1987.

[60] J.-M. Gambaudo, P. Glendinning, and T. Tresser. The gluing bifurcation: symbolic dynamics of the closed curves. Nonlinearity, 1:203-14, 1988.

[61] J.-M. Gambaudo, O.E. Lanford III, and C. Tresser. Dynamique symbolique des rotations. C. R. Acad. Sc. Paris, série I, 299:823-826, 1984.

[62] J.-M. Gambaudo, I. Procaccia, S. Thomae, and C. Tresser. New Universal Scenarios for the Onset of Chaos in Lorenz-Type Flows. Phys. Rev. Lett, 57:925-928, 1986.

[63] J.-M. Gambaudo and C. Tresser. On the dynamics of quasi-contractions. BOL. SOC. BRAS. MAT., 19:61-114, 1988.

[64] L. Gardini, V. Avrutin, and I. Sushko. Codimension-2 border collision, bifurcations in one-dimensional, discontinuous piecewise smooth maps. Int. Journ. Bif. Chaos, 24(2), 2014.

[65] L. Gardini and F. Tramontana. Border Collision Bifurcations in 1D PWL map with one discontinuity and negative jump. Use of the first return map. Int. J. Bifurcation Chaos, 20(11):3529-3547, 2010.

[66] R. Ghrist and P. J. Holmes. Knotting within the gluing bifurcation. In J. Thompson and S. Bishop, editors, IUTAM Symposium on Nonlinearilty and Chaos in the Enginnering Dynamics, pages 299-315. John Wiley Press, 1994. 
[67] D. Giaouris, S. Banerjee, O. Imrayed, K. Mandal, B. Zahawi, and V. Pickert. Complex interaction between tori and onset of three-frequency quasiperiodicity in a current mode controlled boost converter. IEEE Trans. Circ. Syst. I, 59(1):207-214, 2012.

[68] P. Glendinning. Topological conjugation of lorenz maps to $\beta$ transformations. Math. Proc. Camb. Phil. Soc., 107:401-413, 1990.

[69] P. Glendinning. Milnor attractors and topological attractors of a piecewise linear map. Nonlinearity, 14:239-257, 2001.

[70] P. Glendinning and C. Sparrow. Prime and renormalisable kneading invariants and the dynamics of expanding lorenz maps. In Proceedings of a NATO advanced research workshop held at the Centre for Nonlinear Phenomena and Complex Systems on Homoclinic Chaos, pages 22-50, Amsterdam, The Netherlands, The Netherlands, 1993. Elsevier North-Holland, Inc.

[71] A. Granados, S.J. Hogan, and T.M. Seara. The Melnikov method and subharmonic orbits in a piecewise smooth system. SIAM J. Appl. Dyn. Syst., 11:801-830, 2012.

[72] A. Granados, S.J. Hogan, and T.M. Seara. The scattering map in two coupled piecewise-smooth systems, with numerical application to rocking blocks. Physica D, 269:1-20, 2014.

[73] A. Granados and M. Krupa. Firing-rate, symbolic dynamics and frequency dependence in periodically driven spiking models: a piecewise-smooth approach. Nonlinearity (accepted for publication), 2015.

[74] A. Granados, M. Krupa, and F. Clément. Border collision bifurcations of stroboscopic maps in periodically driven spiking models. SIAM J. Appl. Dyn. Syst., 13(4):1387-1416, 2014.

[75] J. Guckenheimer and R. F. Williams. Structural Stability of Lorenz Attractors. Publ. Math. IHES, 50:307-320, 1979.

[76] D. C. Hamill, J. H. B. Deane, and D. J. Jefferies. Modeling of chaotic DC-DC converters by iterated nonlinear mappings. IEEE Trans. Power Electron., 7:25-36, 1992.

[77] G.H. Hardy and E.M. Wright. An Introduction to the Theory of Numbers. Oxford University Press, London, fourth edition, 1960.

[78] S. J. Hogan. On the dynamics of rigid block motion under harmonic forcing. Proc. Roy. Soc. Lond. A, 425:441-476, 1989.

[79] S.J. Hogan, L. Higham, and T.C.L. Griffin. Dynamics of a piecewise linear map with a gap. Proc. Royal Society A, 2006. 
[80] A.J. Homburg. Global aspects of homoclinic bifurcations of vector fields. Memories of the American Math. Soc., 578, 1996.

[81] A.J. Homburg. Piecewise smooth interval maps with non-vanishing derivative. Ergod. Th. \& Dynam. Sys., 20:749-773, 2000.

[82] A.J. Homburg and B. Krauskopf. Resonant homoclinic flip bifurcations. J. Dynam. Differential Equations, 12:807-50, 2000.

[83] E. M. Izhikevich. Dynamical Systems in Neuroscience: The Geometry of Excitability and Bursting. 2007.

[84] N.D Jimenez, S. Mihalas, R. Brown, E. Niebur, and J. Rubin. Locally contractive dynamics in generalized integrate-and-fire neurons. SIAM J. Appl. Dyn. Syst. (SIADS), 12:1474-1514, 2013.

[85] T. Kabe, S. Parui, H. Torikai, S. Banerjee, and T. Saito. Analysis of Piecewise Constant Models of Current Mode Controlled DC-DC Converters. IEICI Trans. Fundamentals, E90-A(2):448, 2007.

[86] K. Kaneko. On the period-adding phenomena at the frequency locking in a one-dimensional mapping. Prog. Theor. Phys., 68:669, 1982.

[87] S. Kapat, S. Banerjee, and A. Patra. Discontinuous map analysis of a dcdc converter governed by pulse skipping modulation. IEEE Transactions on Circuits and Systems I: Regular Papers, 57(7):1793 -1801, 2010.

[88] J.P. Keener. Chaotic behavior on piecewise continuous difference equations. Trans. Am. Math. Soc., 261(2), 1980.

[89] J.P. Keener, F.C Hoppensteadt, and J. Rinzel. Integrate-and-fire models of nerve membrane response to oscillatory input. SIAM J. Appl. Dyn. Syst. (SIADS), 41:503-517, 1981.

[90] M. P. Kennedy and L. O. Chua. Van der Pol and Chaos. IEEE Trans. Circ. 85 Sys., CAS-33, 1986.

[91] C. Kopf. Symbol sequences and entropy for piecewise monotone transformations with discontinuities. Discr. Cont. Dyn. Systs., 6:299-304, 2000.

[92] C. Kopf. Coding and entropy for piecewise continuous piecewise monotone transformations. Nonlinear Analysis, 61:169-275, 2005.

[93] P. Kowalczyk. Robust chaos and border-collision bifurcations in noninvertible piecewise-linear maps. Nonlinearity, 18(2):485, 2005.

[94] P. Kowalczyk, M. di Bernardo, A. R. Champneys, S. J. Hogan, M. Homer, Yu. A. Kuznetsov, A. Nordmark, and P. Piiroinen. Two-parameter nonsmooth bifurcations of limit cycles: classification and open problems. Int. J. Bifurcation Chaos, 16, 2006. 
[95] N.N. Leonov. On a pointwise mapping of a line into itself. Radiofisika, 2(6):942-956, 1959. (in Russian).

[96] N.N. Leonov. On a discontinuous piecewise-linear pointwise mapping of a line into itself. Radiofisika, 3(3):496-510, 1960. (in Russian).

[97] N.N. Leonov. On the theory of a discontinuous mapping of a line into itself. Radiofisika, 3(5):872-886, 1960. (in Russian).

[98] N.N. Leonov. On a discontinuous pointwise mapping of a line into itself. Dok. Akad. Nauk SSSR, 143(5):1038-1041, 1962.

[99] M. Levi. A period-adding phenomenon. SIAM J. Appl. Math., 50(4):943$955,1990$.

[100] D. V. Lyubimov, A. S. Pikovsky, and M. A. Zaks. Universal Scenarios of Transitions to Chaos via Homoclinic Bifurcations, volume 8 of Math. Phys. Rev. Harwood Academic, London, 1989. Russian version 1986 as a Preprint (192) of Russian Academy of Science, Institute of mechanics of solid matter, Sverdlovsk.

[101] O. Makarenkov and J.S.W. Lamb. Dynamics and bifurcations of nonsmooth systems: A survey. Physica D, 241:1826-1844, 2012.

[102] Y. Matsuoka and T. Saito. Rotation Map with a Controlling Segment and Its Application to A/D Converters. IEICI Trans. Fundamentals, E91$\mathrm{A}(7): 1725-1732,2008$.

[103] X. Meng, G. Huguet, and J. Rinzel. Type III excitability, slope sensitivity and coincidence detection. Disc. Cont. Dyn. Syst., 32:2720-2757, 2012.

[104] C. Mira. Chaotic Dynamics: From The One-Dimensional Endomorphism To The Two-Dimensional Diffeomorphism. World Scientific, 1987.

[105] M. Misiurevicz and W. Szlenk. Entropy of piecewise monotone mappings. Studia Mathematica, 67:45-63, 1980.

[106] O. Mondragón-Palomino, T. Danino, J. Selimkhanov, L. Tsimring, and J. Hasty. Entrainment of a population of synthetic genetic oscillators. Science, 333:1315-1319, 2011.

[107] Zbigniew Nitecki. Differentiable Dynamics: Introduction to the Orbit Structure of Diffeormorphisms. MIT Press, 1971.

[108] A. Nordmark and P. Kowalczyk. A codimension-two scenario of sliding solutions in grazing-sliding bifurcations. Nonlinearity, 19:1-26, 2006.

[109] A. B. Nordmark. Existence of periodic orbits in grazing bifurcations of impacting mechanical oscillators. Nonlinearity, 14(6):1517, 2001. 
[110] H. Poincaré. Mémoire sur les courbes définiés par une équation differentielle. J. de Math., 7:375-422, 1981.

[111] E. Ponce, J. Ros, and E. Vela. Unfolding the fold-hopf bifurcation in piecewise linear continuous differential systems with symmetry. Physica $D, 250: 34-46,2013$.

[112] I. Procaccia, S. Thomae, and C. Tresser. First-return maps as a unified renormalization scheme for dynamical systems. Phys. Rev. A, 35:18841900, 1987.

[113] B. Rakshit, M. Apratim, and S. Banerjee. Bifurcation phenomena in twodimensional piecewise smooth discontinuous maps. Chaos, 3, 2010.

[114] F. Rhodes and C.L. Thompson. Rotation numbers for monotone functions on the circle. J. London Math. Soc., 34:360-368, 1986.

[115] T. Saito, T. Kabe, Y. Ishikawa, Y. Matsuoka, and H. Torikai. Piecewise constant switched dynamical systems in power electronics. Int. J. Bifurcation Chaos, 17(10):3373-3386, 2007.

[116] J. Signereska-Rynkowska, J. Touboul, and A. Vidal. A geometric mechanism for mixed-mode bursting oscillations in a hybrid neuron model. Preprint availavle at http://http://arxiv.org/abs/1509.08282, 2015.

[117] D. Simpson. Simultaneous border-collision and period-doubling bifurcations. Chaos, 19(3), 2009.

[118] D.J.W. Simpson. Border-collision bifurcations in $\mathbb{R}^{n}$. To appear in SIAM Review. Preprint available at http://arxiv.org/abs/1407.1895, 2015.

[119] D.J.W. Simpson and J.D. Meiss. Neimark-Sacker Bifurcations in Planar, Piecewise-Smooth, Continuous Maps. SIAM J. Applied Dynamical Systems, 7(3):795-824, 2008.

[120] C. Sparrow. The Lorenz Equations: Bifurcations, Chaos, and Strange Attractors. Springer-Verlag, 1982.

[121] G. Swiatek. Endpoints of rotation intervals for maps of the circle. Erg. Theo. Dyn. Syst., 9(1):173-190, 1989.

[122] P.H.E. Tiesinga, J.-M. Fellous, and T.J. Sejnowski. Spike-time reliability of periodically driven integrate-and-fire neurons. Neurocomputing, 44:195200, 2002.

[123] A. Tonnelier. Threshold curve for the excitability of bidimensional spiking neurons. Phys. Rev. E, 90, 2014.

[124] J. Touboul and R. Brette. Dynamics and bifurcations of the adaptive exponential integrate-and-fire model. Biol. Cybernet, 99:319-334, 2008. 
[125] J. Touboul and R. Brette. Spiking dynamics of bidimensional integrateand-fire neurons. SIAM J. Appl. Dyn. Syst. (SIADS), 4:1462-1506, 2009.

[126] F. Tramontana, L. Gardini, and F. Westerhoff. Heterogeneous speculators and asset price dynamics: further results from a one-dimensional discontinuous piecewise-linear model. Computational Economics, 38:329-347, 2011.

[127] F. Tramontana, F. Westerhoff, and L. Gardini. On the complicated price dynamics of a simple one-dimensional discontinuous financial market model with heterogeneous interacting traders. Journ. Econ. Behav. Organ., 74(3):187-205, 2010.

[128] D. V. Turaev and L. P. Shil'nikov. On bifurcations of a homoclinic "Figure of Eight" for a saddle with a negative saddle value. Soviet Math. Dokl., 34:397-401, 1987 (Russian version 1986).

[129] V.I. Utkin. Variable structure systems with sliding modes. IEEE Trans. on Autom. Cont., 22(2):212-222, 1977.

[130] V.I. Utkin. Sliding mode control design principles and applications to electric drives. IEEE Trans. on Indust. Electr., 40(1):23-36, 1993.

[131] P. Veerman. Irrational rotation numbers. Nonlinearity, 2:419-428, 1989.

[132] M. A. Zaks. Scaling properties and renormalization invariants for the "homoclinic quasiperiodicity". Physica D, 62:300-316, 1993.

[133] Zh. T. Zhusubaliyev and E. Mosekilde. Bifurcations and Chaos in piecewise-smooth dynamical systems, volume 44 of Nonlinear Science A. World Scientific, 2003.

[134] Zh. T. Zhusubaliyev, E. Mosekilde, S. De, and S. Banerjee. Transitions from phase-locked dynamics to chaos in a piecewise-linear map. Phys. Rev. E, 77(026206):1-11, 2008.

[135] Zh. T. Zhusubaliyev, E. A. Soukhoterin, V. N. Rudakov, Y. V. Kolokolov, and E. Mosekilde. Bifurcations and chaotic oscillations in an automatic control relay system with hysteresis. Int. J. Bifurcation Chaos, 11:11931231, 2001.

[136] Z.T. Zhusubaliyev, E. Mosekilde, A.I. Andriyanov, and G.Y. Mikhal'chenko. High-feedback Operation of Power Electronic Converters. Electronics, 2:113-167, 2013. 